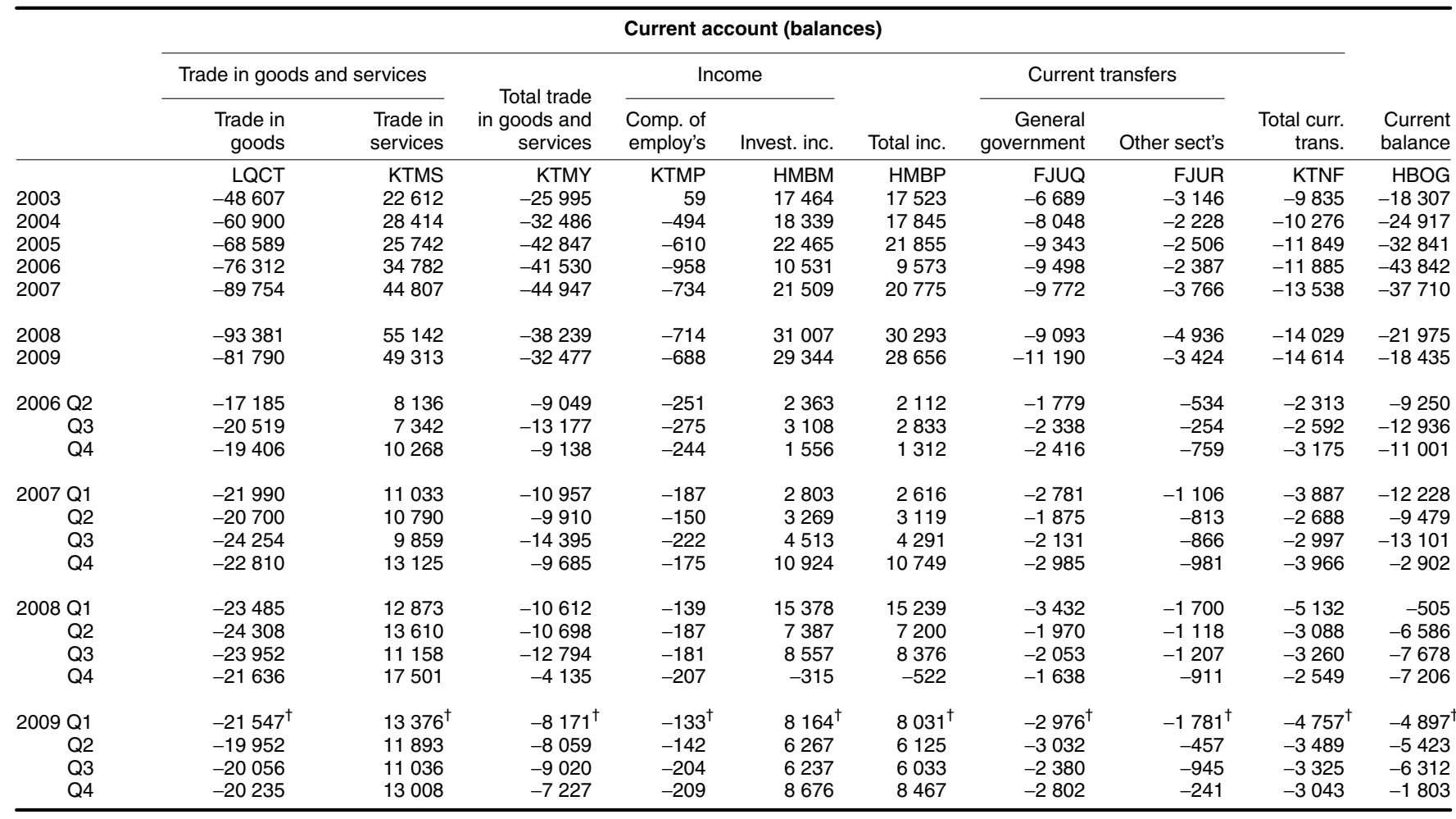

Financial account

\begin{tabular}{|c|c|c|c|c|c|}
\hline & Capital account (balance) & $\begin{array}{r}\text { Trans.in UK assets(net } \\
\text { debits) }\end{array}$ & $\begin{array}{r}\text { Trans.in UK liabilities } \\
\text { (net credits) }\end{array}$ & $\begin{array}{r}\text { Net trans.(net cred. less } \\
\text { net deb's) }\end{array}$ & $\begin{array}{r}\text { Net err's and omissions } \\
\text { (bal.item) }{ }^{1}\end{array}$ \\
\hline & FKMJ & -HBNR & HBNS & HBNT & $\mathrm{HHDH}$ \\
\hline 2003 & 1466 & 341400 & 363953 & 22553 & -5712 \\
\hline 2004 & 2064 & 525815 & 555173 & 29358 & -6505 \\
\hline 2005 & 1503 & 687320 & 716344 & 29024 & 2314 \\
\hline 2006 & 975 & 571859 & 610084 & 38225 & 4642 \\
\hline 2007 & 2566 & 995677 & 1027353 & 31676 & 3468 \\
\hline 2008 & 3241 & -614196 & -599014 & 15182 & 3552 \\
\hline 2006 Q2 & -630 & -9223 & 6531 & 15754 & -5874 \\
\hline Q3 & 483 & 122722 & 129579 & 6857 & 5596 \\
\hline Q4 & 515 & 117498 & 125472 & 7974 & 2512 \\
\hline 2007 Q1 & 364 & 445604 & 453624 & 8020 & 3844 \\
\hline Q2 & 456 & 199992 & 205534 & 5542 & 3481 \\
\hline Q3 & 626 & 159562 & 169725 & 10163 & 2312 \\
\hline Q4 & 1120 & 190519 & 198470 & 7951 & -6169 \\
\hline Q2 & 981 & -369333 & -368082 & 1251 & 4354 \\
\hline Q3 & 585 & 26751 & 24855 & -1896 & 8989 \\
\hline Q4 & 748 & -565833 & -552457 & 13376 & -6918 \\
\hline 2009 Q1 & 832 & $-124142^{\dagger}$ & $-118472^{\dagger}$ & $5670^{\dagger}$ & $-1605^{\dagger}$ \\
\hline Q2 & 950 & -17363 & -17555 & -192 & 4665 \\
\hline Q3 & $875^{\dagger}$ & -15300 & -16702 & -1402 & 6839 \\
\hline Q4 & 972 & -15793 & -9644 & 6149 & -5318 \\
\hline
\end{tabular}

1 This series represents net errors and omissions in the balance of payments

Source: National Statistics accounts and is the sum of the current and capital balances (HBOG and

FKMJ) and net financial account transactions (HBNT) with reversal of sign. 
$£$ million

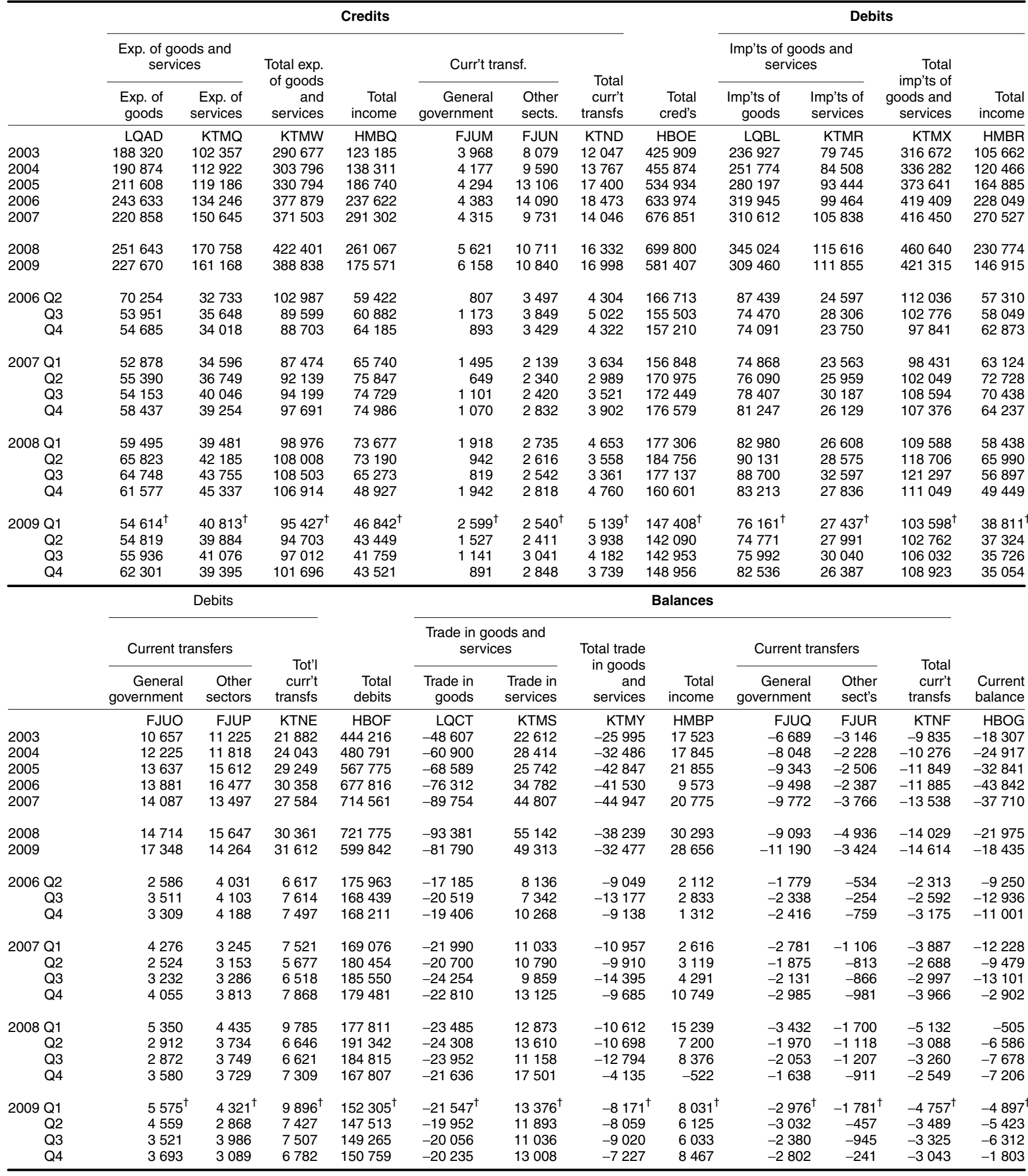


$£$ million

\begin{tabular}{|c|c|c|c|c|c|c|c|c|c|}
\hline & \multicolumn{3}{|c|}{ Credits } & \multicolumn{3}{|c|}{ Debits } & \multicolumn{3}{|c|}{ Balances } \\
\hline & $\begin{array}{l}\text { Capital } \\
\text { transfers }\end{array}$ & $\begin{array}{r}\text { Acquisition of } \\
\text { non-produced, } \\
\text { non-financial } \\
\text { assets }\end{array}$ & Total credits & $\begin{array}{l}\text { Capital } \\
\text { transfers }\end{array}$ & $\begin{array}{r}\text { Disposals of } \\
\text { non-produced, } \\
\text { non-financial } \\
\text { assets }\end{array}$ & Total debits & $\begin{array}{l}\text { Capital } \\
\text { transfers }\end{array}$ & $\begin{array}{r}\text { Non-produced, } \\
\text { non-financial } \\
\text { assets }\end{array}$ & Capital balance \\
\hline & FJMX & FJUX & FKMH & FJMY & FJUY & FKMI & FJMZ & NHSG & FKMJ \\
\hline 2003 & 2575 & 218 & 2793 & 1038 & 289 & 1327 & 1537 & -71 & 1466 \\
\hline 2004 & 3409 & 193 & 3602 & 1026 & 512 & 1538 & 2383 & -319 & 2064 \\
\hline 2005 & 3973 & 337 & 4310 & 2212 & 595 & 2807 & 1761 & -258 & 1503 \\
\hline 2006 & 3393 & 631 & 4024 & 2426 & 623 & 3049 & 967 & 8 & 975 \\
\hline 2007 & 3810 & 783 & 4593 & 1233 & 794 & 2027 & 2577 & -11 & 2566 \\
\hline 2008 & 4589 & 1074 & 5663 & 1308 & 1114 & 2422 & 3281 & -40 & 3241 \\
\hline 2009 & 4251 & 1603 & 5854 & 1339 & 886 & 2225 & 2912 & 717 & 3629 \\
\hline 2006 Q2 & 757 & 179 & 936 & 1403 & 163 & 1566 & -646 & 16 & -630 \\
\hline Q3 & 818 & 150 & 968 & 341 & 144 & 485 & 477 & 6 & 483 \\
\hline Q4 & 864 & 149 & 1013 & 331 & 167 & 498 & 533 & -18 & 515 \\
\hline 2007 Q1 & 870 & 108 & 978 & 431 & 183 & 614 & 439 & -75 & 364 \\
\hline Q2 & 783 & 140 & 923 & 262 & 205 & 467 & 521 & -65 & 456 \\
\hline Q3 & 835 & 239 & 1074 & 270 & 178 & 448 & 565 & 61 & 626 \\
\hline Q4 & 1322 & 296 & 1618 & 270 & 228 & 498 & 1052 & 68 & 1120 \\
\hline 2008 Q1 & 1363 & 287 & 1650 & 419 & 304 & 723 & 944 & -17 & 927 \\
\hline Q2 & 1231 & 288 & 1519 & 257 & 281 & 538 & 974 & 7 & 981 \\
\hline Q3 & 900 & 210 & 1110 & 280 & 245 & 525 & 620 & -35 & 585 \\
\hline Q4 & 1095 & 289 & 1384 & 352 & 284 & 636 & 743 & 5 & 748 \\
\hline 2009 Q1 & 1006 & 448 & 1454 & 436 & 186 & 622 & 570 & 262 & 832 \\
\hline Q2 & 1110 & 360 & 1470 & 261 & 259 & 520 & 849 & 101 & 950 \\
\hline Q3 & 1074 & $376^{\dagger}$ & $1450^{\dagger}$ & 304 & $271^{\dagger}$ & $575^{\dagger}$ & 770 & $105^{\dagger}$ & $875^{\dagger}$ \\
\hline Q4 & 1061 & 419 & 1480 & 338 & 170 & 508 & 723 & 249 & 972 \\
\hline
\end{tabular}




\begin{tabular}{|c|c|c|c|c|c|c|c|c|c|c|c|}
\hline & \multicolumn{10}{|c|}{ Financial account (net transactions) } & \multirow[b]{3}{*}{ Total } \\
\hline & \multicolumn{4}{|c|}{ Direct investment } & \multicolumn{3}{|c|}{ Portfolio investment } & \multirow[b]{2}{*}{$\begin{array}{r}\text { Financial } \\
\text { derivatives }\end{array}$} & \multirow[b]{2}{*}{$\begin{array}{r}\text { Other } \\
\text { investment }\end{array}$} & \multirow[b]{2}{*}{$\begin{array}{r}\text { Reserve } \\
\text { assets }\end{array}$} & \\
\hline & $\begin{array}{l}\text { Equity } \\
\text { capital }\end{array}$ & $\begin{array}{r}\text { Reinvested } \\
\text { earnings }\end{array}$ & $\begin{array}{r}\text { Other } \\
\text { capital } \\
\text { transactions }\end{array}$ & $\begin{array}{r}\text { Total net } \\
\text { direct } \\
\text { investment }\end{array}$ & $\begin{array}{r}\text { Equity } \\
\text { securities }\end{array}$ & $\begin{array}{r}\text { Debt } \\
\text { securities }\end{array}$ & $\begin{array}{r}\text { Total net } \\
\text { portfolio } \\
\text { investment }\end{array}$ & & & & \\
\hline & HBWN & HBWT & HBWU & HJYV & HBWV & HBWX & $\mathrm{HHZD}$ & ZPNN & HHYR & LTCV & HBNT \\
\hline 2003 & -16175 & -14027 & 6089 & -24113 & 404 & 68976 & 69380 & -5401 & -18872 & 1559 & 22553 \\
\hline 2004 & 3820 & -22518 & -1604 & -20302 & -54690 & 11020 & -43670 & -7875 & 101401 & -196 & 29358 \\
\hline 2005 & 67337 & -33054 & 19557 & 53840 & -54944 & 33038 & -21906 & 9556 & -11810 & -656 & 29024 \\
\hline 2006 & 28574 & -25683 & 37000 & 39891 & -30161 & 45243 & 15082 & 7449 & -24623 & 426 & 38225 \\
\hline 2007 & 24945 & -34591 & -28334 & -37980 & -16031 & 127284 & 111253 & -19001 & -21405 & -1191 & 31676 \\
\hline 2008 & 8154 & -30165 & -15831 & -37842 & 98459 & 210894 & 309353 & -35509 & -222158 & 1338 & 15182 \\
\hline 2009 & 18692 & -15244 & 14020 & 17468 & 30590 & 3313 & 33903 & 14450 & -49833 & -5763 & 10225 \\
\hline 2006 Q2 & 10510 & -6134 & 24586 & 28962 & -7930 & 6222 & -1708 & 174 & -11884 & 210 & 15754 \\
\hline Q3 & 6745 & -5898 & 8907 & 9754 & -1237 & 52580 & 51343 & -2101 & -51754 & -385 & 6857 \\
\hline Q4 & -8439 & -6113 & -14231 & -28783 & -1174 & -23342 & -24516 & 10203 & 50934 & 136 & 7974 \\
\hline 2007 Q1 & 8648 & -8039 & 6431 & 7040 & -20620 & -8064 & -28684 & -6057 & 34897 & 824 & 8020 \\
\hline Q2 & 11069 & -7616 & -9356 & -5903 & -7306 & 44153 & 36847 & -2522 & -22813 & -67 & 5542 \\
\hline Q3 & 8916 & -9051 & -9455 & -9590 & -16571 & 58771 & 42200 & -3537 & -19468 & 558 & 10163 \\
\hline Q4 & -3688 & -9885 & -15954 & -29527 & 28466 & 32424 & 60890 & -6885 & -14021 & -2506 & 7951 \\
\hline 2008 Q1 & 14928 & -14699 & 5466 & 5695 & 30441 & 38012 & 68453 & -29379 & -43249 & 931 & 2451 \\
\hline Q2 & 2860 & -9827 & -6987 & -13954 & 43313 & 63703 & 107016 & 10941 & -102644 & -108 & 1251 \\
\hline Q3 & -2617 & -16079 & -2067 & -20763 & -4489 & 26779 & 22290 & 11375 & -16930 & 2132 & -1896 \\
\hline Q4 & -7017 & 10440 & -12243 & -8820 & 29194 & 82400 & 111594 & -28446 & -59335 & -1617 & 13376 \\
\hline 2009 Q1 & $-788^{\dagger}$ & $-5661^{\dagger}$ & $867^{\dagger}$ & $-5582^{\dagger}$ & $37489^{\dagger}$ & $21142^{\dagger}$ & $58631^{\dagger}$ & 2137 & $-51393^{\dagger}$ & $1877^{\dagger}$ & $5670^{\dagger}$ \\
\hline Q2 & -886 & -10213 & 4944 & -6155 & 11065 & -6030 & 5035 & 1871 & 361 & -1304 & -192 \\
\hline Q3 & -1032 & 573 & 11312 & 10853 & -16268 & -5090 & -21358 & $5109^{\dagger}$ & 10781 & -6787 & -1402 \\
\hline Q4 & 21398 & 57 & -3103 & 18352 & -1696 & -6709 & -8405 & 5333 & -9582 & 451 & 6149 \\
\hline
\end{tabular}

International Investment Position (net balance sheets)

\begin{tabular}{|c|c|c|c|c|c|c|c|c|c|}
\hline & \multicolumn{3}{|c|}{ Direct investment } & \multicolumn{3}{|c|}{ Portfolio investment } & \multirow[b]{2}{*}{$\begin{array}{r}\text { Other } \\
\text { investment }\end{array}$} & \multirow[b]{2}{*}{ Reserve assets } & \multirow[b]{2}{*}{ Total } \\
\hline & $\begin{array}{r}\text { Equity capital } \\
\text { and reinvested } \\
\text { earnings }\end{array}$ & Other capital & $\begin{array}{r}\text { Total net } \\
\text { direct } \\
\text { investment }\end{array}$ & $\begin{array}{r}\text { Equity } \\
\text { securities }\end{array}$ & Debt securities & $\begin{array}{r}\text { Total net } \\
\text { portfolio } \\
\text { investment }\end{array}$ & & & \\
\hline & HBSH & CGKF & HBWQ & CGNE & CGNF & CGNH & CGNG & LTEB & HBQC \\
\hline 2003 & 424991 & -89412 & 335579 & -155101 & 8057 & -147044 & -329479 & 23794 & -117150 \\
\hline 2004 & 399312 & -104570 & 294742 & -119447 & -16374 & -135821 & -402863 & 23250 & -220692 \\
\hline 2005 & 358068 & -146383 & 211685 & -42139 & -58629 & -100768 & -388248 & 24739 & -252592 \\
\hline 2006 & 337767 & -174386 & 163381 & -86603 & -84921 & -171524 & -367348 & 22931 & -352560 \\
\hline 2007 & 392096 & -108327 & 283769 & -65091 & -158721 & -223812 & -369162 & 26685 & -282520 \\
\hline 2008 & 478398 & -100337 & 378061 & -28676 & -252376 & -281052 & -192478 & 36283 & -59186 \\
\hline 2009 & 439404 & -114243 & 325161 & -113480 & -336925 & -450405 & -97514 & 40144 & -182614 \\
\hline 2006 Q2 & 346026 & -178580 & 167446 & -89071 & -52616 & -141687 & -366820 & 23634 & -317427 \\
\hline Q3 & 328730 & -188894 & 139836 & -79408 & -109108 & -188516 & -315517 & 23624 & -340573 \\
\hline Q4 & 337767 & -174386 & 163381 & -86603 & -84921 & -171524 & -367348 & 22931 & -352560 \\
\hline 2007 Q1 & 403434 & -146243 & 257191 & -106794 & -55862 & -162656 & -406329 & 22414 & -289380 \\
\hline Q4 & 392096 & -108327 & 283769 & -65091 & -158721 & -223812 & -369162 & 26685 & -282520 \\
\hline 2008 Q1 & 382662 & -122412 & 260250 & -67301 & -209771 & -277072 & -322497 & 28171 & -311148 \\
\hline Q2 & 400514 & -114656 & 285858 & -85269 & -292788 & -378057 & -226617 & 27555 & -291261 \\
\hline Q3 & 431935 & -112359 & 319576 & -54811 & -286466 & -341277 & -215397 & 27030 & -210068 \\
\hline Q4 & 478398 & -100337 & 378061 & -28676 & -252376 & -281052 & -192478 & 36283 & -59186 \\
\hline 2009 Q1 & $473965^{\dagger}$ & $-101039^{\dagger}$ & $372926^{\dagger}$ & $-64492^{\dagger}$ & $-248625^{\dagger}$ & $-313117^{\dagger}$ & $-130818^{\dagger}$ & 34181 & $-36828^{\dagger}$ \\
\hline Q2 & 435547 & -106063 & 329484 & -91408 & -323543 & -414951 & -112250 & 31686 & -166031 \\
\hline Q3 & 442350 & -117524 & 324826 & -127790 & -321710 & -449500 & -108432 & 40949 & -192157 \\
\hline Q4 & 439404 & -114243 & 325161 & -113480 & -336925 & -450405 & -97514 & 40144 & -182614 \\
\hline
\end{tabular}


$£$ million

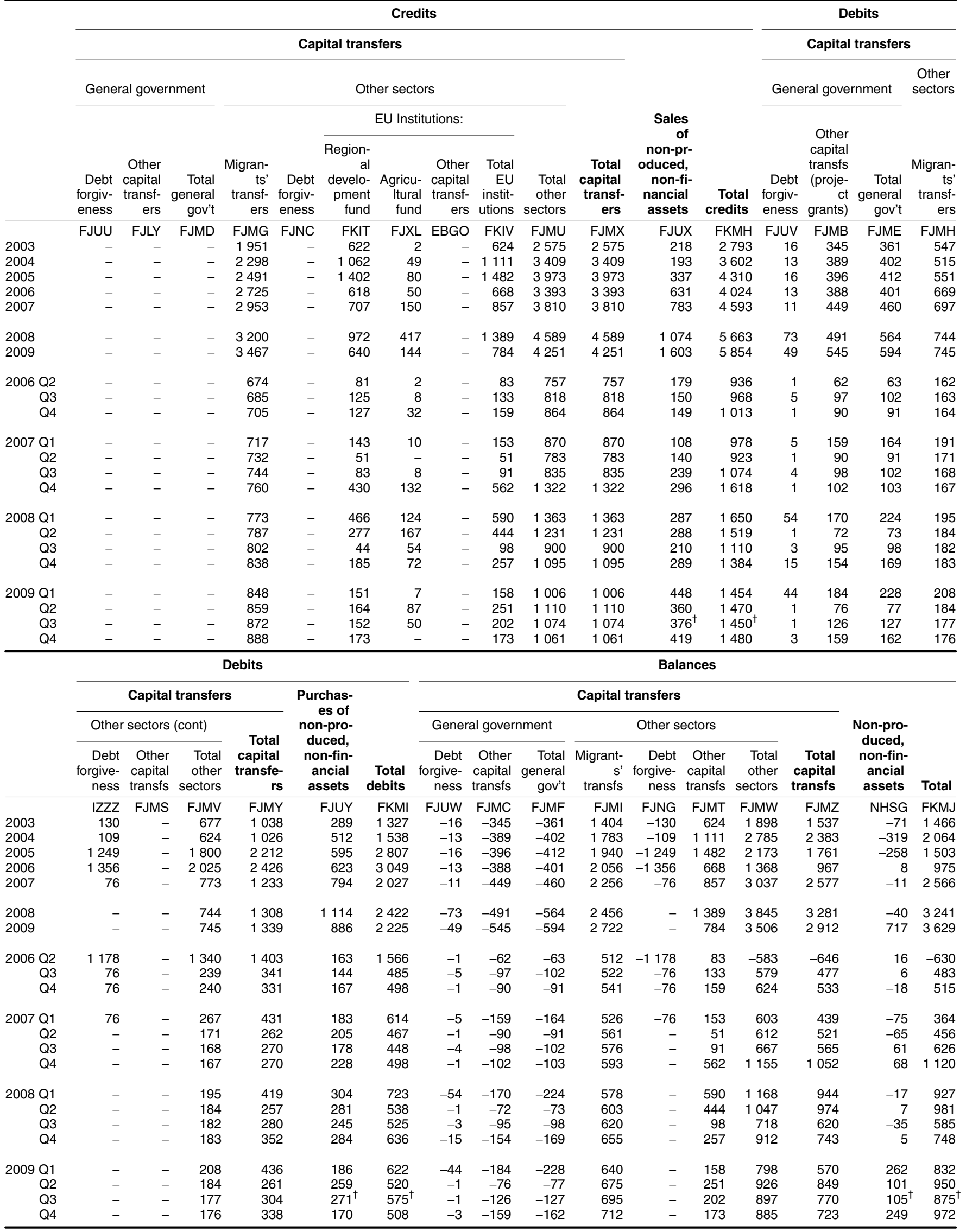


$£$ million

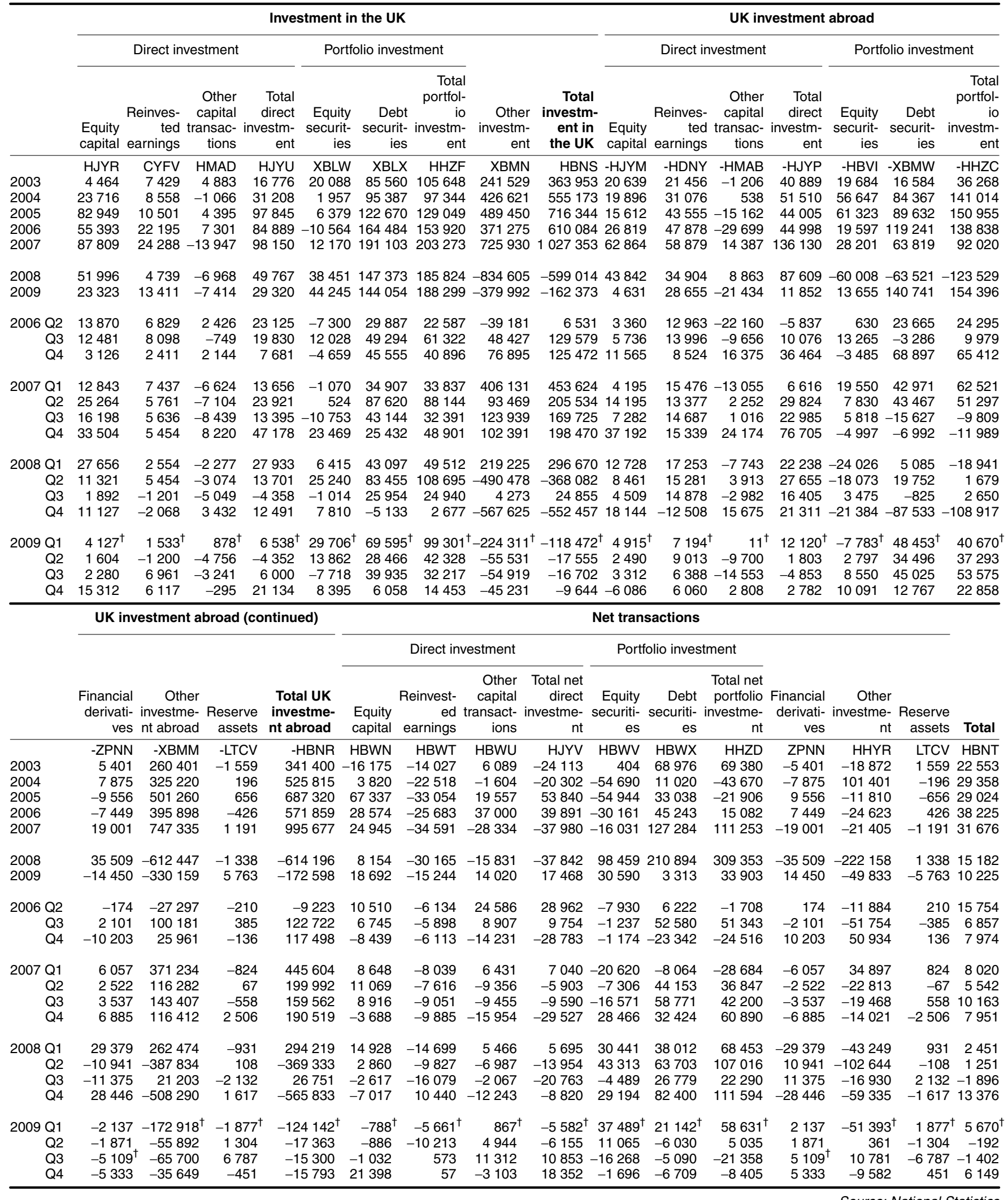




\begin{tabular}{|c|c|c|c|c|c|c|c|c|c|c|c|c|c|}
\hline & \multicolumn{6}{|c|}{ UK investment abroad (net debits) } & \multicolumn{6}{|c|}{ UK investment abroad (net credits) } & \multirow[b]{3}{*}{$\begin{array}{r}\text { total } \\
\text { investment } \\
\text { in the UK }\end{array}$} \\
\hline & \multicolumn{6}{|c|}{ By: } & \multirow[b]{2}{*}{$\begin{array}{c}\text { Tot'I UK } \\
\text { invest. } \\
\text { abroad }\end{array}$} & \multicolumn{5}{|c|}{ In: } & \\
\hline & Monetary fir & $\begin{array}{l}\text { Buil. } \\
\text { soc's }\end{array}$ & $\begin{array}{r}\text { Tot'l } \\
\text { monet'y } \\
\text { fin. } \\
\text { instit's }\end{array}$ & $\begin{array}{l}\text { Cen'l } \\
\text { gov't }\end{array}$ & Pub. corps & $\begin{array}{r}\text { Other } \\
\text { sectors }\end{array}$ & & $\begin{array}{r}\text { UK } \\
\text { Monetary } \\
\text { Financial } \\
\text { Instituti- } \\
\text { ons(MFIs) }\end{array}$ & $\begin{array}{r}\text { Central } \\
\text { government }\end{array}$ & $\begin{array}{r}\text { Local } \\
\text { authoriti- } \\
\text { es }\end{array}$ & $\begin{array}{r}\text { Public } \\
\text { corporati- } \\
\text { ons }\end{array}$ & $\begin{array}{l}\text { Other } \\
\text { sectors }\end{array}$ & \\
\hline & -HFAM & HEQN & -HFAQ & -HFAN & -HFAO & -HFAP & -HBNR & CGUL & HFAR & HFAS & HFAT & GGCJ & HBNS \\
\hline 2003 & 181513 & -1786 & 179727 & -2217 & -571 & 164461 & 341400 & 204512 & 13657 & 204 & - & 145580 & 363953 \\
\hline 2004 & 326315 & 868 & 327183 & 1103 & -370 & 197899 & 525815 & 340046 & 13526 & 651 & 283 & 200667 & 555173 \\
\hline 2005 & 396393 & -2230 & 394163 & 1588 & -1671 & 293240 & 687320 & 321335 & 29688 & 134 & -7 & 365194 & 716344 \\
\hline 2006 & 427338 & 2649 & 429987 & 630 & -4694 & 145936 & 571859 & 428038 & 26219 & 232 & -12 & 155607 & 610084 \\
\hline 2007 & 690726 & 2969 & 693695 & 4698 & -18 & 297302 & 995677 & 777460 & 28810 & -42 & -6 & 221131 & 1027353 \\
\hline 2008 & -467969 & -222 & -468191 & 1987 & -599 & -147393 & -614196 & -526927 & 37472 & 446 & -9 & -109996 & -599014 \\
\hline 2009 & -272557 & 861 & -271696 & 8619 & 70 & 90409 & -172598 & -199510 & 27663 & 520 & -4 & 8958 & -162373 \\
\hline 2006 Q2 & 40650 & 947 & 41597 & 226 & -1460 & -49586 & -9223 & 62987 & 4238 & 153 & -3 & -60844 & 6531 \\
\hline Q3 & 115519 & 728 & 116247 & 133 & -325 & 6667 & 122722 & 82719 & 6586 & -8 & - & 40282 & 129579 \\
\hline Q4 & 46171 & 602 & 46773 & 514 & -2456 & 72667 & 117498 & 45261 & 10820 & 69 & -3 & 69325 & 125472 \\
\hline 2007 Q1 & 321407 & -603 & 320804 & -1065 & -67 & 125932 & 445604 & 351619 & 607 & -17 & - & 101415 & 453624 \\
\hline Q2 & 130092 & 702 & 130794 & 704 & 26 & 68468 & 199992 & 82585 & 18239 & -94 & -3 & 104807 & 205534 \\
\hline Q3 & 129139 & 701 & 129840 & 3317 & 42 & 26363 & 159562 & 189603 & 1300 & 30 & - & -21208 & 169725 \\
\hline Q4 & 110088 & 2169 & 112257 & 1742 & -19 & 76539 & 190519 & 153653 & 8664 & 39 & -3 & 36117 & 198470 \\
\hline 2008 Q1 & 139929 & 493 & 140422 & 2772 & -496 & 151521 & 294219 & 160265 & 1721 & 329 & -1 & 134356 & 296670 \\
\hline Q2 & -282158 & 427 & -281731 & -6381 & 187 & -81408 & -369333 & -337708 & 16190 & -41 & -4 & -46519 & -368082 \\
\hline Q3 & 56659 & 1813 & 58472 & 4742 & -270 & -36193 & 26751 & 110659 & 23391 & 20 & -1 & -109214 & 24855 \\
\hline Q4 & -382399 & -2955 & -385354 & 854 & -20 & -181313 & -565833 & -460143 & -3830 & 138 & -3 & -88619 & -552457 \\
\hline & $-32884^{\dagger}$ & 31 & $-32853^{\dagger}$ & $-1670^{\dagger}$ & 23 & $-89642^{\dagger}$ & $-124142^{\dagger}$ & $-32549^{\dagger}$ & $14514^{\dagger}$ & 195 & -1 & $-100631^{\dagger}$ & $-118472^{\dagger}$ \\
\hline Q2 & -102338 & 972 & -101366 & 5367 & $-7^{\dagger}$ & 78643 & -17363 & -64738 & -918 & 71 & -2 & 48032 & -17555 \\
\hline Q3 & -96347 & 25 & -96322 & 5843 & -13 & 75192 & -15300 & -75783 & 456 & 196 & -1 & 58430 & -16702 \\
\hline & -40988 & -167 & -41155 & -921 & 67 & 26216 & -15793 & -26440 & 13611 & 58 & - & 3127 & -9644 \\
\hline & & & & & & Net tra & ansactions & & & & & & \\
\hline & & & & & & In assets a & and liabilities & s of: & & & & & \\
\hline & & $\begin{array}{r}\text { Mo } \\
\text { (bks }\end{array}$ & $\begin{array}{l}\text { net'y fin. in } \\
\text { and buil. so }\end{array}$ & & Cent'l go & ov't & Loc. auth's & & ub. corps. & Oth. & sects. & Net tr & ransactions \\
\hline & & & $\mathrm{GG}$ & CK & $\mathrm{HF}$ & $\mathrm{AV}$ & HFAS & & HFAW & & GGCL & & HBNT \\
\hline 2003 & & & 247 & 785 & 158 & 374 & 204 & & 571 & & 18881 & & 22553 \\
\hline 2004 & & & 128 & 363 & 124 & 423 & 651 & & 653 & & 2768 & & 29358 \\
\hline 2005 & & & -728 & 328 & 281 & 100 & 134 & & 1664 & & 71954 & & 29024 \\
\hline 2006 & & & $-1 \mathrm{~s}$ & 949 & 255 & 589 & 232 & & 4682 & & 9671 & & 38225 \\
\hline 2007 & & & 837 & 765 & 241 & 12 & -42 & & 12 & & 76171 & & 31676 \\
\hline 2008 & & & -587 & 736 & 354 & 185 & 446 & & 590 & & 37397 & & 15182 \\
\hline 2009 & & & 721 & 186 & 190 & 44 & 520 & & -74 & & 81451 & & 10225 \\
\hline 2006 Q2 & & & 213 & 390 & 40 & 12 & 153 & & 1457 & & 11258 & & 15754 \\
\hline Q3 & & & -335 & 528 & 64 & 453 & -8 & & 325 & & 33615 & & 6857 \\
\hline Q4 & & & -15 & 512 & 103 & 306 & 69 & & 2453 & & -3342 & & 7974 \\
\hline 2007 Q1 & & & 308 & 315 & 16 & 372 & -17 & & 67 & & 24517 & & 8020 \\
\hline Q2 & & & -482 & 209 & 175 & 535 & -94 & & -29 & & 36339 & & 5542 \\
\hline Q3 & & & 597 & 763 & -20 & 17 & 30 & & -42 & & 47571 & & 10163 \\
\hline Q4 & & & 413 & 396 & 69 & 22 & 39 & & 16 & & 40422 & & 7951 \\
\hline 2008 Q1 & & & 198 & 343 & -10 & 51 & 329 & & 495 & & 17165 & & 2451 \\
\hline Q2 & & & -559 & 977 & 225 & 571 & -41 & & -191 & & 34889 & & 1251 \\
\hline Q3 & & & 521 & 187 & 186 & 849 & 20 & & 269 & & 73021 & & -1896 \\
\hline Q4 & & & -747 & 789 & -46 & 884 & 138 & & 17 & & 92694 & & 13376 \\
\hline 2009 Q1 & & & & $304^{\dagger}$ & 161 & $184^{\dagger}$ & 195 & & -24 & & $10989^{\dagger}$ & & $5670^{\dagger}$ \\
\hline Q2 & & & 366 & 628 & -62 & 85 & 71 & & $5^{\dagger}$ & & 30611 & & -192 \\
\hline Q3 & & & 205 & 539 & -53 & 387 & 196 & & 12 & & 16762 & & -1402 \\
\hline Q4 & & & 147 & 715 & 145 & 532 & 58 & & -67 & & 23089 & & 6149 \\
\hline
\end{tabular}




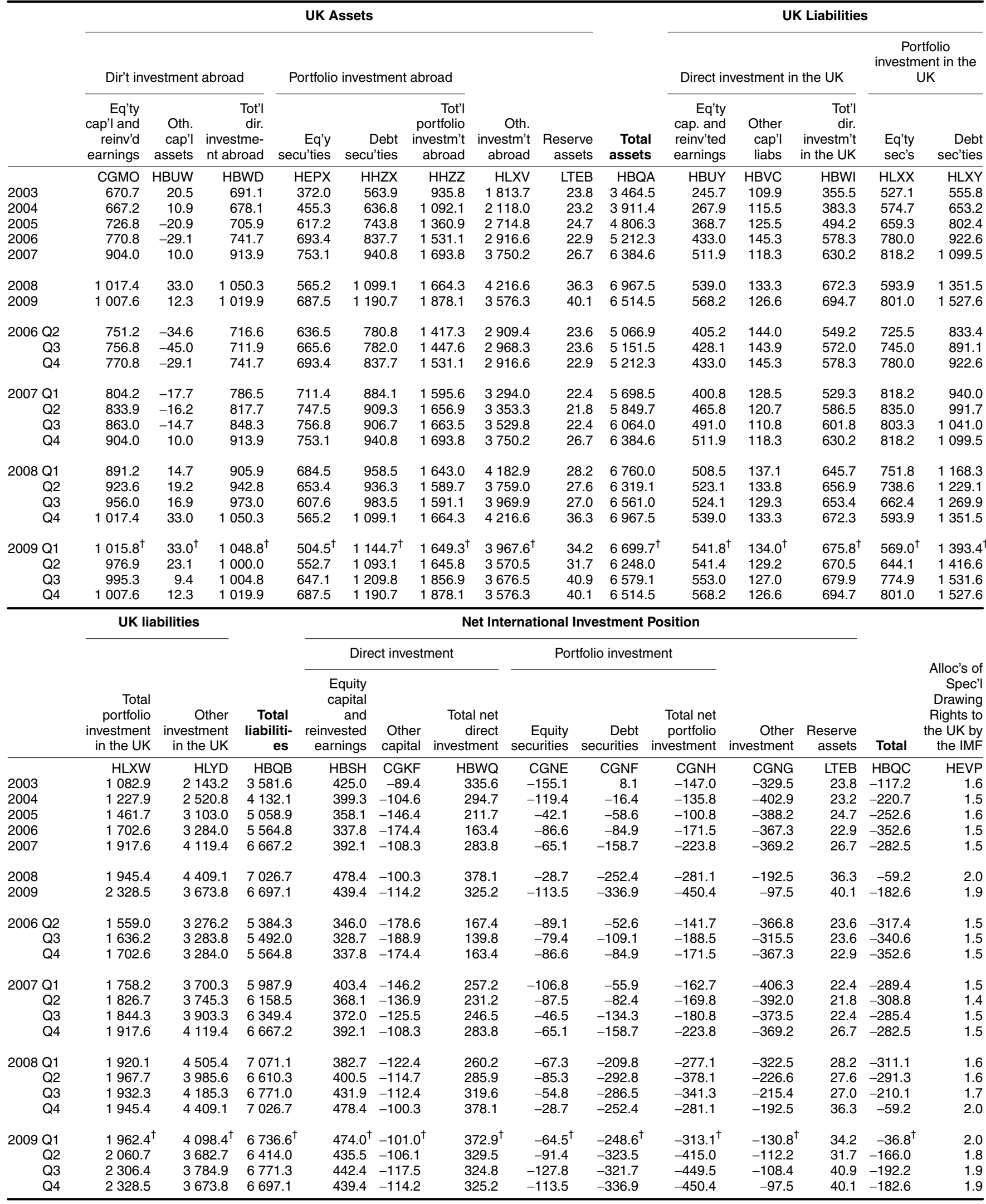




\begin{tabular}{|c|c|c|c|c|c|c|c|c|c|c|c|c|c|}
\hline & & & UK Asse & & & & & & UK I & _iabilities & & & \\
\hline & Monetary & inancial i & stitutions & & & & & UK Monet'y & & & & & \\
\hline & Banks & $\begin{array}{l}\text { Buil. } \\
\text { socs. }\end{array}$ & $\begin{array}{r}\text { Tot'l } \\
\text { monet'y } \\
\text { fin. } \\
\text { insts. }\end{array}$ & $\begin{array}{r}\text { Cent'l } \\
\text { gov't }\end{array}$ & $\begin{array}{l}\text { Pub. } \\
\text { corps. }\end{array}$ & $\begin{array}{l}\text { Oth. } \\
\text { sects. }\end{array}$ & $\begin{array}{r}\text { Total } \\
\text { assets }\end{array}$ & $\begin{array}{r}\text { insts. } \\
\text { (bks \& } \\
\text { buil. } \\
\text { socs) }\end{array}$ & $\begin{array}{r}\text { Cent'l } \\
\text { gov't }\end{array}$ & $\begin{array}{l}\text { Loc. } \\
\text { auth's }\end{array}$ & $\begin{array}{l}\text { Pub. } \\
\text { corps. }\end{array}$ & $\begin{array}{l}\text { Oth. } \\
\text { sect's }\end{array}$ & $\begin{array}{c}\text { Total } \\
\text { liabiliti- } \\
\text { es }\end{array}$ \\
\hline & CGNI & VTXF & CGNJ & CGNK & CGNL & CGNM & HBQA & HBYJ & CGOG & $\mathrm{CGOH}$ & CGOI & $\mathrm{HCON}$ & HBQB \\
\hline 2003 & 1730637 & 7267 & 1737904 & 34233 & 4214 & 1688101 & 3464452 & 1893346 & 70883 & 1083 & 16 & 1616274 & 3581602 \\
\hline 2004 & 1983458 & 7755 & 1991213 & 33386 & 4670 & 1882126 & 3911395 & 2179991 & 89317 & 1734 & 461 & 1860584 & 4132087 \\
\hline 2005 & 2420539 & 7714 & 2428253 & 35764 & 3354 & 2338950 & 4806321 & 2582365 & 115211 & 1866 & 446 & 2359025 & 5058913 \\
\hline 2006 & 2674909 & 10358 & 2685267 & 35000 & 1742 & 2490264 & 5212273 & 2817933 & 141223 & 2097 & 429 & 2603151 & 5564833 \\
\hline 2007 & 3460940 & 13328 & 3474268 & 39866 & 1839 & 2868660 & 6384633 & 3694956 & 167608 & 2054 & 421 & 2802114 & 6667153 \\
\hline 2008 & 3945875 & 18461 & 396 & & & & & & & & 413 & & 7026728 \\
\hline 2009 & & 18275 & & & & & & & & & 396 & 2798543 & 6697079 \\
\hline 2006 Q2 & 2600197 & 9029 & 2609226 & & 2966 & 2419373 & & & & & 434 & 2470947 & 5384297 \\
\hline Q3 & 2684619 & 9754 & 2694373 & 35043 & 2630 & 2419407 & 5151453 & 2840497 & 131165 & 2029 & 433 & 2517902 & 5492026 \\
\hline 2007 Q1 & 2998804 & 9754 & 3008558 & 34318 & 1759 & 2653837 & 5698472 & 3172856 & 139234 & 2080 & 428 & 2673254 & 5987852 \\
\hline Q2 & 3083971 & 10456 & 3094427 & 34012 & 1800 & 2719488 & 5849727 & 3212739 & 152617 & 1985 & 424 & 2790754 & 6158519 \\
\hline Q3 & 3252137 & 11160 & 3263297 & 35060 & 1857 & 2763814 & 6064028 & 3433784 & 157679 & 2015 & 423 & 2755498 & 6349399 \\
\hline Q4 & 3460940 & 13328 & 3474268 & 39866 & 1839 & 2868660 & 6384633 & 3694956 & 167608 & 2054 & 421 & 2802114 & 6667153 \\
\hline 2008 Q1 & 3715204 & 17740 & 3732944 & & 13 & & 9992 & 3998516 & 17 & 2383 & 421 & & 1140 \\
\hline Q2 & 3391576 & 18140 & 3409716 & 40426 & 1821 & 2867090 & 9053 & 3630164 & 180082 & 2342 & 418 & 2797308 & 6610314 \\
\hline Q3 & 3623865 & 19581 & 3643446 & 40688 & 1515 & 2875323 & & 3906059 & 209260 & & 417 & 2652942 & 6771040 \\
\hline Q4 & 3945875 & 18461 & 3964336 & 53002 & 1555 & 2948649 & 6967542 & 4163032 & 223117 & 2500 & 413 & 2637666 & 7026728 \\
\hline 2009 Q1 & $3829306^{\dagger}$ & 17724 & $3847030^{\dagger}$ & $49082^{\dagger}$ & 1574 & $2802061^{\dagger}$ & $6699747^{\dagger}$ & $4042299^{\dagger}$ & 243153 & 2695 & $406^{\dagger}$ & $2448022^{\dagger}$ & $6736575^{\dagger}$ \\
\hline Q2 & 3387621 & 17837 & 3405458 & 47543 & 1365 & 2793598 & 6247964 & 3647521 & $239500^{\dagger}$ & 2766 & 404 & 2523804 & 6413995 \\
\hline Q3 & 3472654 & 19549 & 3492203 & 57231 & 1399 & 3028293 & 6579126 & 3736926 & 240324 & 2962 & 404 & 2790667 & 6771283 \\
\hline Q4 & 3364379 & 18275 & 3382654 & 57020 & 1386 & 3073405 & 6514465 & 3649216 & 245904 & 3020 & 396 & 2798543 & 6697079 \\
\hline
\end{tabular}

Net International Investment Position

\begin{tabular}{|c|c|c|c|c|c|c|}
\hline & & & & & & \multirow[b]{2}{*}{ Net transactions } \\
\hline & $\begin{array}{l}\text { Monet'y fin. insts. } \\
\text { (bks \& buil. socs) }\end{array}$ & Cent'l gov't & Loc. auth's & Pub. corps & Oth' sects. & \\
\hline & HDIJ & CGOK & $-\mathrm{CGOH}$ & CGOL & HDKB & HBQC \\
\hline 2003 & -155442 & -36650 & -1083 & 4198 & 71827 & -117150 \\
\hline 2005 & -154112 & -79447 & -1866 & 2908 & -20075 & -252592 \\
\hline 2006 & -132666 & -106223 & -2097 & 1313 & -112887 & -352560 \\
\hline 2007 & -220688 & -127742 & -2054 & 1418 & 66546 & -282520 \\
\hline 2008 & -198696 & -170115 & -2500 & 1142 & 310983 & -59186 \\
\hline 2009 & -266562 & -188884 & -3020 & 990 & 274862 & -182614 \\
\hline 2006 Q2 & -181087 & -85261 & -2037 & 2532 & -51574 & -317427 \\
\hline Q3 & -146124 & -96122 & -2029 & 2197 & -98495 & -340573 \\
\hline Q4 & -132666 & -106223 & -2097 & 1313 & -112887 & -352560 \\
\hline 2007 Q1 & -164298 & -104916 & -2080 & 1331 & -19417 & -289380 \\
\hline Q2 & -118312 & -118605 & -1985 & 1376 & -71266 & -308792 \\
\hline Q3 & -170487 & -122619 & -2015 & 1434 & 8316 & -285371 \\
\hline Q4 & -220688 & -127742 & -2054 & 1418 & 66546 & -282520 \\
\hline 2008 Q1 & -265572 & -131564 & -2383 & 898 & 87473 & -311148 \\
\hline Q2 & -220448 & -139656 & -2342 & 1403 & 69782 & -291261 \\
\hline Q3 & -262613 & -168572 & -2362 & 1098 & 222381 & -210068 \\
\hline Q4 & -198696 & -170115 & -2500 & 1142 & 310983 & -59186 \\
\hline 2009 Q1 & $-195269^{\dagger}$ & $-194071^{\dagger}$ & -2695 & $1168^{\dagger}$ & $354039^{\dagger}$ & $-36828^{\dagger}$ \\
\hline Q2 & -242063 & -191957 & -2766 & 961 & 269794 & -166031 \\
\hline Q3 & -244723 & -183093 & -2962 & 995 & 237626 & -192157 \\
\hline Q4 & -266562 & -188884 & -3020 & 990 & 274862 & -182614 \\
\hline
\end{tabular}




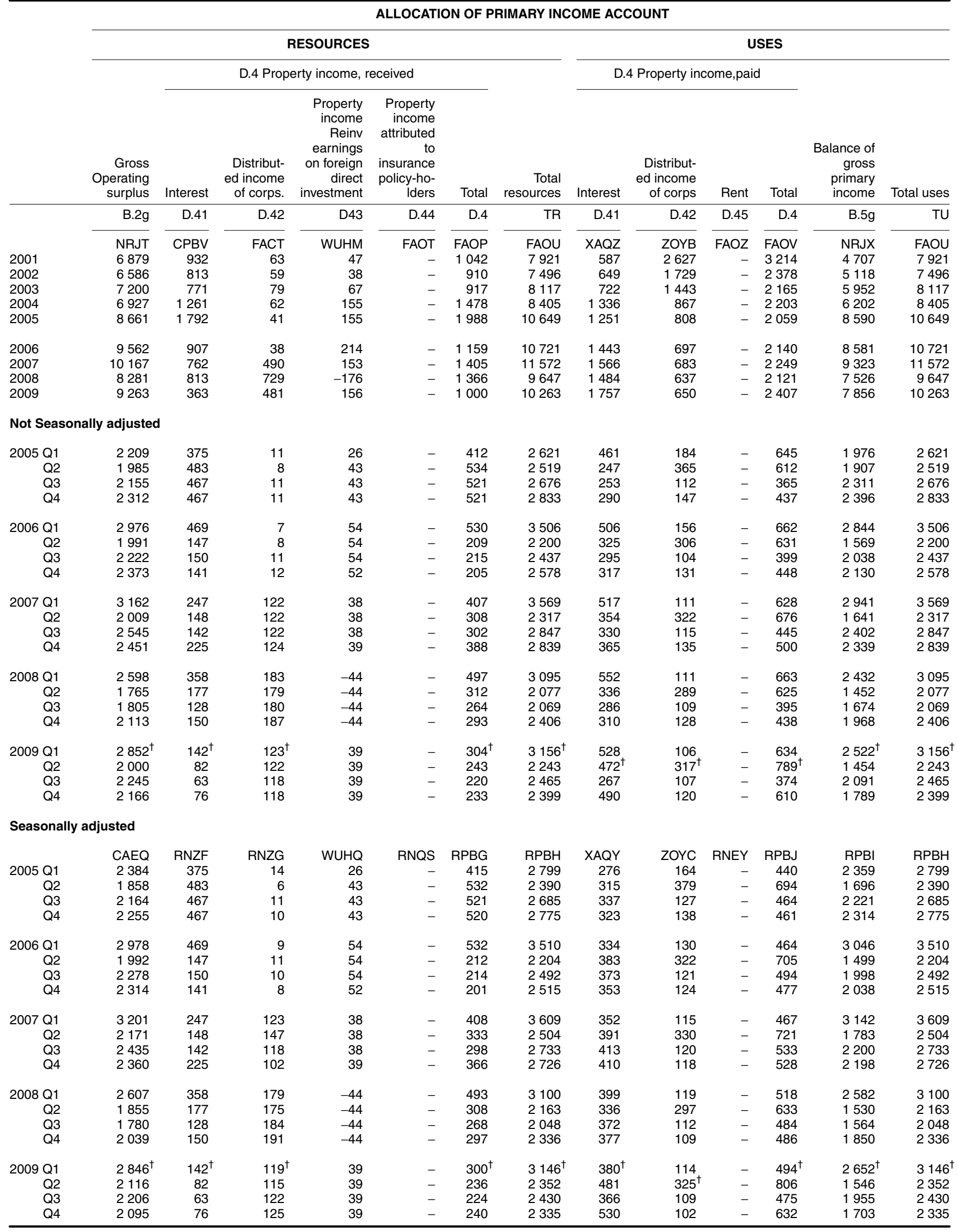




\begin{tabular}{|c|c|c|c|c|c|c|c|c|c|c|c|}
\hline & \multicolumn{11}{|c|}{ SECONDARY DISTRIBUTION OF INCOME ACCOUNT } \\
\hline & \multicolumn{5}{|c|}{ RESOURCES } & \multicolumn{6}{|c|}{ USES } \\
\hline & $\begin{array}{r}\text { Balance of } \\
\text { gross } \\
\text { primary } \\
\text { incomes }\end{array}$ & $\begin{array}{r}\text { Imputed } \\
\text { social } \\
\text { contributio- } \\
\text { ns }\end{array}$ & $\begin{array}{r}\text { Net non-life } \\
\text { insurance } \\
\text { claims }\end{array}$ & $\begin{array}{r}\text { Miscellaneo- } \\
\text { us current } \\
\text { transfers }\end{array}$ & $\begin{array}{r}\text { Total } \\
\text { resources }\end{array}$ & $\begin{array}{c}\text { Taxes on } \\
\text { income }\end{array}$ & $\begin{array}{r}\text { Social } \\
\text { benefits } \\
\text { other than } \\
\text { social } \\
\text { transfers in } \\
\text { kind }\end{array}$ & $\begin{array}{r}\text { Net non-life } \\
\text { insurance } \\
\text { premiums }\end{array}$ & $\begin{array}{r}\text { Miscellaneo- } \\
\text { us current } \\
\text { transfers }\end{array}$ & $\begin{array}{r}\text { Gross } \\
\text { Disposable } \\
\text { income }\end{array}$ & Total uses \\
\hline & B.5g & D.612 & D.72 & D75 & TR & D.51 & D.623 & D.71 & D75 & B.6g & TU \\
\hline & NRJX & EWRS & FDDF & CY89 & FDDH & FCCS & EWRS & FDDM & $\mathrm{CX} 6 \mathrm{C}$ & NRKD & $\mathrm{FDDH}$ \\
\hline 2001 & 4707 & 128 & - & 122 & 4957 & 90 & 128 & - & 95 & 4644 & 4957 \\
\hline 2002 & 5118 & 138 & - & 147 & 5403 & 61 & 138 & - & 58 & 5146 & 5403 \\
\hline 2003 & 5952 & 131 & - & 124 & 6207 & 94 & 131 & - & 28 & 5954 & 6207 \\
\hline 2004 & 6202 & 131 & - & 28 & 6361 & 75 & 131 & - & 5 & 6150 & 6361 \\
\hline 2005 & 8590 & 132 & - & - & 8722 & 141 & 132 & - & - & 8449 & 8722 \\
\hline 2006 & 8581 & 135 & - & - & 8716 & 372 & 135 & - & - & 8209 & 8716 \\
\hline 2007 & 9323 & 137 & - & - & 9460 & 225 & 137 & - & - & 9098 & 9460 \\
\hline 2008 & 7526 & 140 & - & - & 7666 & 254 & 140 & - & - & 7272 & 7666 \\
\hline 2009 & 7856 & 140 & - & - & 7996 & 272 & 140 & - & - & 7584 & 7996 \\
\hline
\end{tabular}

Not Seasonally adjusted

$\begin{array}{rll}2005 \text { Q1 } & 1976 & 33 \\ \text { Q2 } & 1907 & 33 \\ \text { Q3 } & 2311 & 33 \\ \text { Q4 } & 2396 & 33 \\ & & \\ 2006 \text { Q1 } & 2844 & 33 \\ \text { Q2 } & 1569 & 34 \\ \text { Q3 } & 2038 & 34 \\ \text { Q4 } & 2130 & 34 \\ & & \\ 2007 \text { Q1 } & 2941 & 34 \\ \text { Q2 } & 1641 & 35 \\ \text { Q3 } & 2402 & 34 \\ \text { Q4 } & 2339 & 34 \\ & & \\ 2008 \text { Q1 } & 2432 & 35 \\ \text { Q2 } & 1452 & 35 \\ \text { Q3 } & 1674 & 35 \\ \text { Q4 } & 1968 & 35 \\ & & \\ 2009 \text { Q1 } & 2522^{\dagger} & 35 \\ \text { Q2 } & 1454 & 35 \\ \text { Q3 } & 2091 & 35 \\ \text { Q4 } & 1789 & 35\end{array}$

Seasonally adjusted

\begin{tabular}{|c|c|c|c|c|c|c|c|c|c|c|c|}
\hline & RPBI & & & & RPKN & RPKP & & RNPQ & CY87 & RPKO & RPKN \\
\hline 2005 Q1 & 2359 & 33 & - & - & 2392 & 35 & 33 & - & - & 2324 & 2392 \\
\hline Q2 & 1696 & 33 & - & - & 1729 & 35 & 33 & - & - & 1661 & 1729 \\
\hline Q3 & 2221 & 33 & - & - & 2254 & 35 & 33 & - & - & 2186 & 2254 \\
\hline Q4 & 2314 & 33 & - & - & 2347 & 36 & 33 & - & - & 2278 & 2347 \\
\hline 2006 Q1 & 3046 & 33 & - & - & 3079 & 93 & 33 & - & - & 2953 & 3079 \\
\hline Q2 & 1499 & 34 & - & - & 1533 & 93 & 34 & - & - & 1406 & 1533 \\
\hline Q3 & 1998 & 34 & - & - & 2032 & 93 & 34 & - & - & 1905 & 2032 \\
\hline Q4 & 2038 & 34 & - & - & 2072 & 93 & 34 & - & - & 1945 & 2072 \\
\hline 2007 Q1 & 3142 & 34 & - & - & 3176 & 56 & 34 & - & - & 3086 & 3176 \\
\hline Q2 & 1783 & 35 & - & - & 1818 & 57 & 35 & - & - & 1726 & 1818 \\
\hline Q3 & 2200 & 34 & - & - & 2234 & 56 & 34 & - & - & 2144 & 2234 \\
\hline Q4 & 2198 & 34 & - & - & 2232 & 56 & 34 & - & - & 2142 & 2232 \\
\hline 2008 Q1 & 2582 & 35 & - & - & 2617 & 64 & 35 & - & - & 2518 & 2617 \\
\hline Q2 & 1530 & 35 & - & - & 1565 & 63 & 35 & - & - & 1467 & 1565 \\
\hline Q3 & 1564 & 35 & - & - & 1599 & 64 & 35 & - & - & 1500 & 1599 \\
\hline Q4 & 1850 & 35 & - & - & 1885 & 63 & 35 & - & - & 1787 & 1885 \\
\hline Q2 & 1546 & 35 & - & - & 1581 & 68 & 35 & - & - & 1478 & 1581 \\
\hline Q3 & 1955 & 35 & - & - & 1990 & 68 & 35 & - & - & 1887 & 1990 \\
\hline Q4 & 1703 & 35 & - & - & 1738 & 68 & 35 & - & - & 1635 & 1738 \\
\hline
\end{tabular}




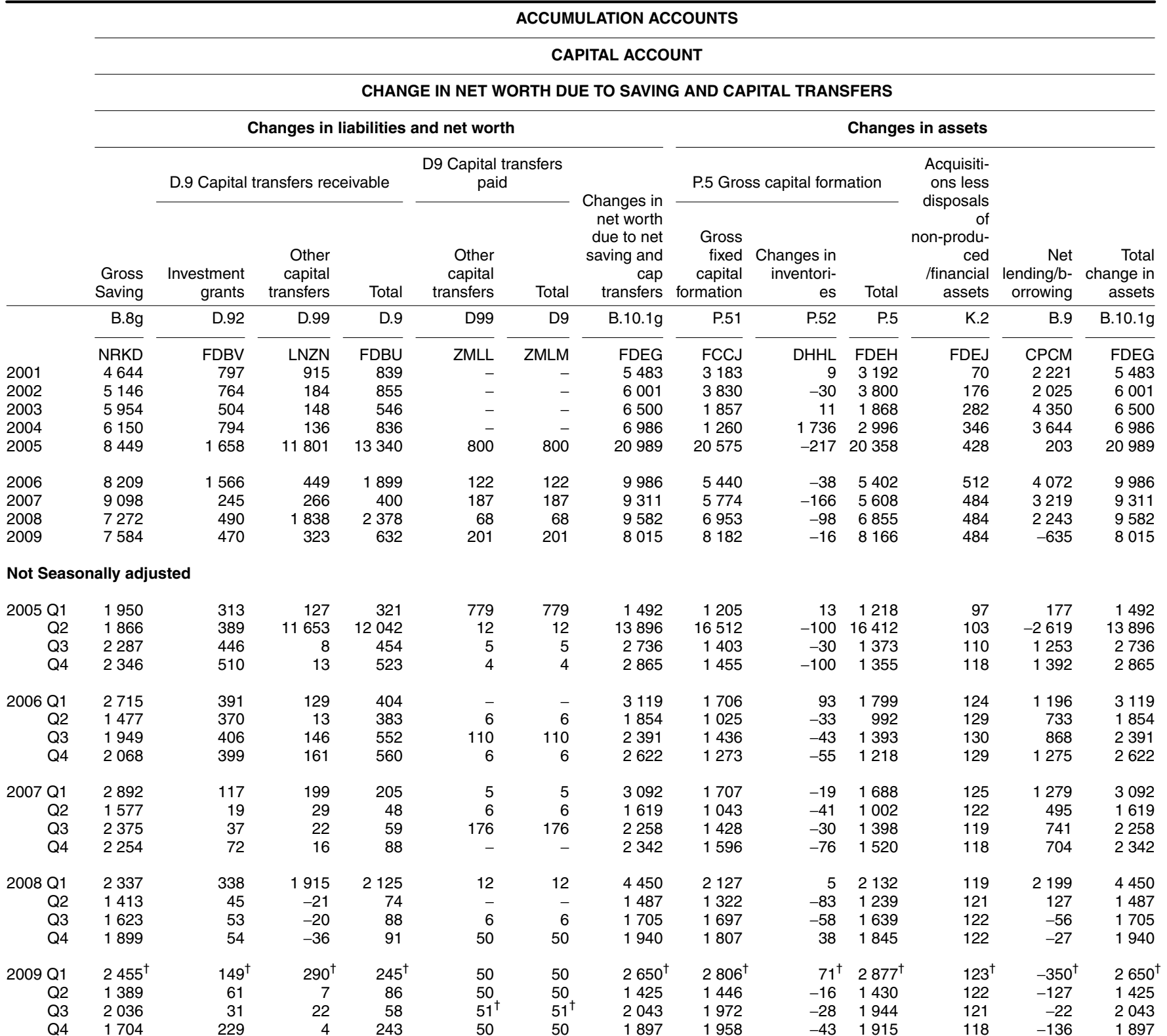

Seasonally adjusted

\begin{tabular}{|c|c|c|c|c|c|c|c|c|c|c|c|c|c|}
\hline & RPKO & RNZT & ROAF & RPXF & ZMLN & ZMLO & RPXD & RNZD & DGAH & RQBR & RQBO & RQBN & RPXD \\
\hline 2005 Q1 & 2324 & 231 & 8 & 239 & 779 & 779 & 1784 & 973 & 13 & 986 & 97 & 701 & 1784 \\
\hline Q2 & 1661 & 558 & 11653 & 12211 & 12 & 12 & 13860 & 16756 & -100 & 16656 & 103 & -2899 & 13860 \\
\hline Q3 & 2186 & 446 & 8 & 454 & 5 & 5 & 2635 & 1422 & -30 & 1392 & 110 & 1133 & 2635 \\
\hline Q4 & 2278 & 423 & 13 & 436 & 4 & 4 & 2710 & 1424 & -100 & 1324 & 118 & 1268 & 2710 \\
\hline 2006 Q1 & 2953 & 303 & 13 & 316 & - & - & 3269 & 1339 & 93 & 1432 & 124 & 1713 & 3269 \\
\hline Q2 & 1406 & 508 & 13 & 521 & 6 & 6 & 1921 & 1289 & -33 & 1256 & 129 & 536 & 1921 \\
\hline Q3 & 1905 & 396 & 146 & 542 & 110 & 110 & 2337 & 1511 & -43 & 1468 & 130 & 739 & 2337 \\
\hline Q4 & 1945 & 359 & 161 & 520 & 6 & 6 & 2459 & 1301 & -55 & 1246 & 129 & 1084 & 2459 \\
\hline 2007 Q1 & 3086 & 108 & 88 & 196 & 5 & 5 & 3277 & 1344 & -19 & 1325 & 125 & 1827 & 3277 \\
\hline Q2 & 1726 & 30 & 29 & 59 & 6 & 6 & 1779 & 1311 & -41 & 1270 & 122 & 387 & 1779 \\
\hline Q3 & 2144 & 39 & 22 & 61 & 176 & 176 & 2029 & 1490 & -30 & 1460 & 119 & 450 & 2029 \\
\hline Q4 & 2142 & 68 & 16 & 84 & - & - & 2226 & 1629 & -76 & 1553 & 118 & 555 & 2226 \\
\hline 2008 Q1 & 2518 & 317 & 1787 & 2104 & 12 & 12 & 4610 & 1687 & 5 & 1692 & 119 & 2799 & 4610 \\
\hline Q2 & 1467 & 69 & 29 & 98 & - & - & 1565 & 1678 & -83 & 1595 & 121 & -151 & 1565 \\
\hline Q3 & 1500 & 58 & 35 & 93 & 6 & 6 & 1587 & 1756 & -58 & 1698 & 122 & -233 & 1587 \\
\hline Q4 & 1787 & 46 & 37 & 83 & 50 & 50 & 1820 & 1832 & 38 & 1870 & 122 & -172 & 1820 \\
\hline Q2 & 1478 & 111 & 25 & 136 & 50 & 50 & 1564 & 1813 & -16 & 1797 & 122 & -355 & 1564 \\
\hline Q3 & 1887 & 38 & 27 & 65 & $51^{\dagger}$ & $51^{\dagger}$ & 1901 & 2021 & -28 & 1993 & 121 & -213 & 1901 \\
\hline Q4 & 1635 & 202 & 14 & 216 & 50 & 50 & 1801 & 2000 & -43 & 1957 & 118 & -274 & 1801 \\
\hline
\end{tabular}




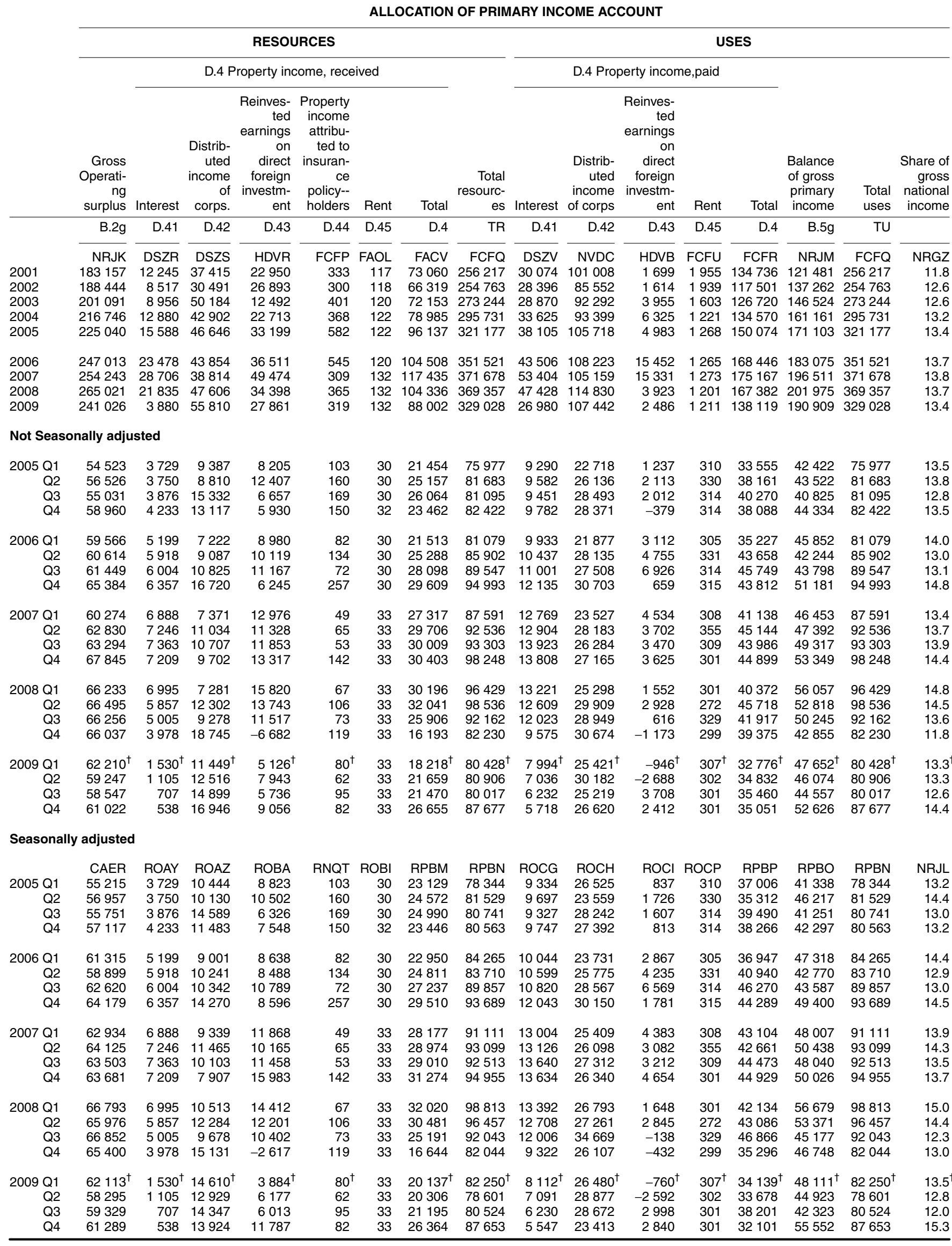


SECONDARY DISTRIBUTION OF INCOME ACCOUNT

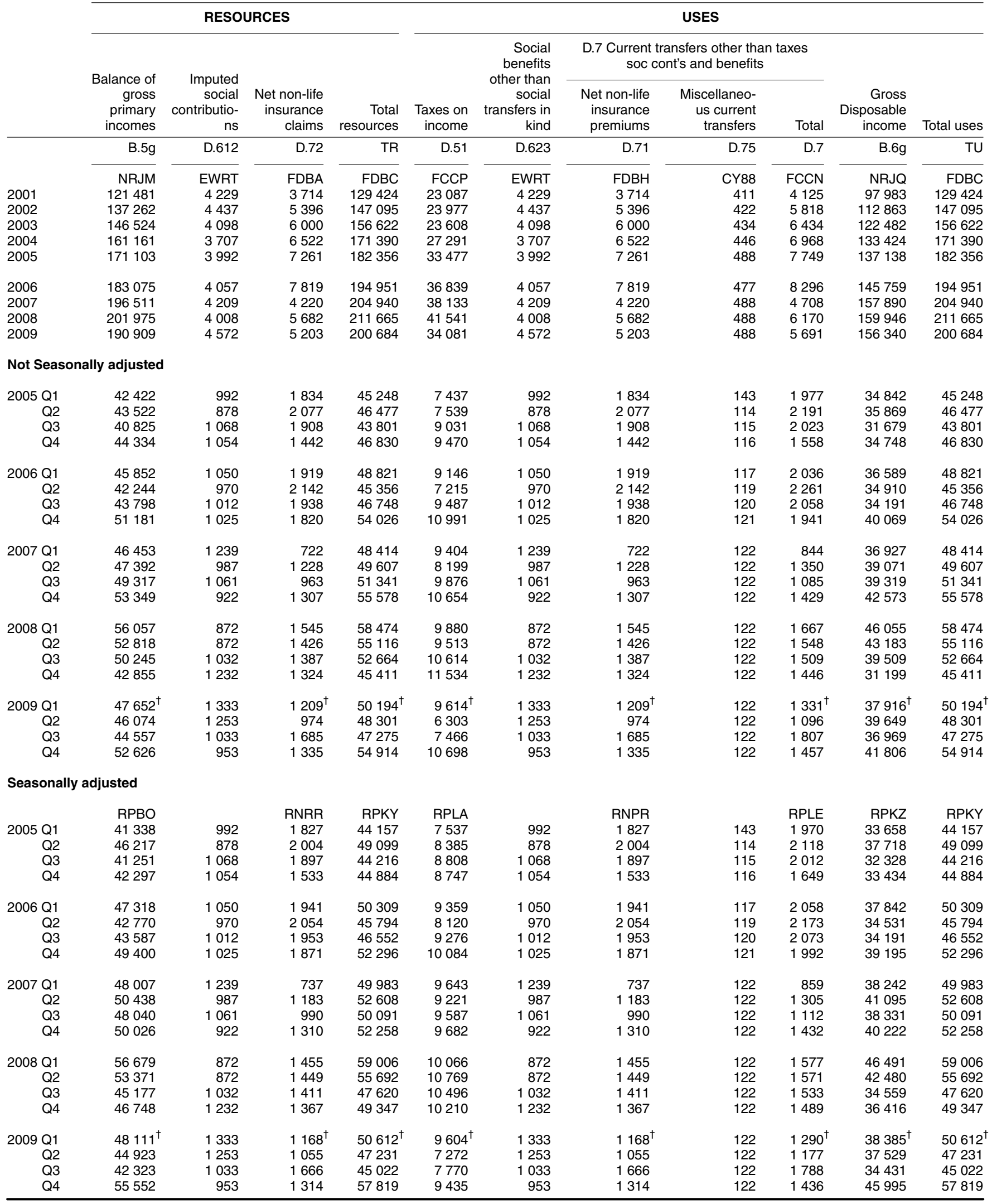




\begin{tabular}{|c|c|c|c|c|c|c|c|c|c|c|c|c|c|c|}
\hline & \multicolumn{14}{|c|}{ ACCUMULATION ACCOUNTS } \\
\hline & \multicolumn{14}{|c|}{ CAPITAL ACCOUNT } \\
\hline & \multicolumn{14}{|c|}{ CHANGE IN NET WORTH DUE TO SAVING AND CAPITAL TRANSFERS } \\
\hline & \multicolumn{7}{|c|}{ Changes in liabilities and net worth } & \multicolumn{7}{|c|}{ Changes in assets } \\
\hline & \multicolumn{6}{|c|}{-D.9 Less capital tranfers } & & \multicolumn{4}{|c|}{ P.5 Gross capital formation } & \multirow{2}{*}{$\begin{array}{r}\text { Acquisit- } \\
\text { ions less } \\
\text { disposals } \\
\text { of } \\
\text { non-prod- } \\
\text { uced } \\
\text { /financi- } \\
\text { al assets }\end{array}$} & \multirow{2}{*}{$\begin{array}{r}\text { Net } \\
\text { lending/- } \\
\text { borrowing }\end{array}$} & \multirow[b]{2}{*}{$\begin{array}{r}\text { Total } \\
\text { change in } \\
\text { assets }\end{array}$} \\
\hline & $\begin{array}{l}\text { Gross I } \\
\text { Saving }\end{array}$ & $\begin{array}{l}\text { Investme- } \\
\text { nt grants }\end{array}$ & $\begin{array}{r}\text { Other } \\
\text { capital } \\
\text { transfers }\end{array}$ & $\begin{array}{c}\text { Capital } \\
\text { taxes }\end{array}$ & $\begin{array}{r}\text { Other } \\
\text { capital } \\
\text { transfers }\end{array}$ & Total & $\begin{array}{r}\text { Total } \\
\text { change in } \\
\text { liabilit- } \\
\text { ies and } \\
\text { net worth }\end{array}$ & $\begin{array}{r}\text { Gross } \\
\text { fixed } \\
\text { capital } \\
\text { formation }\end{array}$ & $\begin{array}{r}\text { Changes } \\
\text { in } \\
\text { inventor- } \\
\text { ies }\end{array}$ & $\begin{array}{r}\text { Acquisit- } \\
\text { ions less } \\
\text { disposals } \\
\text { of } \\
\text { valuables }\end{array}$ & Total & & & \\
\hline & B.8g & D.92 & D.99 & $-\mathrm{D} .91$ & -D.99 & $-\mathrm{D} .9$ & B. $10.1 \mathrm{~g}$ & P.51 & P.52 & P.53 & P.5 & K.2 & B.9 & B.10.1g \\
\hline $\begin{array}{l}2001 \\
2002 \\
2003 \\
2004 \\
2005\end{array}$ & $\begin{array}{r}\text { NRJQ } \\
97983 \\
112863 \\
122482 \\
133424 \\
137138\end{array}$ & $\begin{array}{l}\text { AIBR } \\
3048 \\
3131 \\
5059 \\
4928 \\
5140\end{array}$ & $\begin{array}{r}\text { LNZM } \\
873 \\
93 \\
106 \\
94 \\
119\end{array}$ & $\begin{array}{r}-Q Y K B \\
- \\
- \\
- \\
- \\
-\end{array}$ & $\begin{array}{l}- \text { CISB } \\
-285 \\
-492 \\
-575 \\
-419 \\
-456\end{array}$ & $\begin{array}{r}-F C F X \\
-285 \\
-492 \\
-575 \\
-419 \\
-456\end{array}$ & $\begin{array}{r}\text { NRMG } \\
101619 \\
115595 \\
127072 \\
138027 \\
141941\end{array}$ & $\begin{array}{r}\text { FDBM } \\
103127 \\
103182 \\
103855 \\
105288 \\
108979\end{array}$ & $\begin{array}{l}\text { DLQX } \\
5941 \\
2677 \\
3734 \\
2679 \\
4909\end{array}$ & $\begin{array}{r}\text { NPOV } \\
- \\
-43 \\
-97 \\
-99 \\
-171\end{array}$ & $\begin{array}{r}\text { FDCL } \\
109068 \\
105816 \\
107492 \\
107868 \\
113717\end{array}$ & $\begin{array}{r}\text { FDCN } \\
1138 \\
1255 \\
959 \\
1339 \\
1317\end{array}$ & $\begin{array}{r}\text { DTAL } \\
-8587 \\
8524 \\
18621 \\
28820 \\
26907\end{array}$ & $\begin{array}{r}\text { NRMG } \\
101619 \\
115595 \\
127072 \\
138027 \\
141941\end{array}$ \\
\hline $\begin{array}{l}2006 \\
2007 \\
2008 \\
2009\end{array}$ & $\begin{array}{l}145759 \\
157890 \\
159946 \\
156340\end{array}$ & $\begin{array}{r}6106 \\
7611 \\
6679 \\
10978\end{array}$ & $\begin{array}{l}116 \\
111 \\
-50 \\
161\end{array}$ & $\begin{array}{l}- \\
- \\
- \\
-\end{array}$ & $\begin{array}{r}-508 \\
-660 \\
-722 \\
-1005\end{array}$ & $\begin{array}{r}-508 \\
-660 \\
-722 \\
-1005\end{array}$ & $\begin{array}{l}151473 \\
164952 \\
165853 \\
166474\end{array}$ & $\begin{array}{l}115364 \\
127561 \\
127297 \\
104270\end{array}$ & $\begin{array}{r}4824 \\
6644 \\
1219 \\
-14462\end{array}$ & $\begin{array}{r}-42 \\
-22 \\
26 \\
19\end{array}$ & $\begin{array}{r}120146 \\
134183 \\
128542 \\
89827\end{array}$ & $\begin{array}{r}869 \\
2489 \\
854 \\
176\end{array}$ & $\begin{array}{l}30458 \\
28280 \\
36457 \\
76471\end{array}$ & $\begin{array}{l}151473 \\
164952 \\
165853 \\
166474\end{array}$ \\
\hline \multicolumn{15}{|c|}{ Not Seasonally adjusted } \\
\hline $\begin{array}{r}2005 \text { Q1 } \\
\text { Q2 } \\
\text { Q3 } \\
\text { Q4 }\end{array}$ & $\begin{array}{l}34842 \\
35869 \\
31679 \\
34748\end{array}$ & $\begin{array}{r}1877 \\
1108 \\
933 \\
1222\end{array}$ & $\begin{array}{r}119 \\
- \\
- \\
-\end{array}$ & $\begin{array}{l}- \\
- \\
- \\
-\end{array}$ & $\begin{array}{r}-88 \\
-124 \\
-120 \\
-124\end{array}$ & $\begin{array}{r}-88 \\
-124 \\
-120 \\
-124\end{array}$ & $\begin{array}{l}36750 \\
36853 \\
32492 \\
35846\end{array}$ & $\begin{array}{l}26829 \\
24885 \\
28000 \\
29265\end{array}$ & $\begin{array}{r}2657 \\
-133 \\
3044 \\
-659\end{array}$ & $\begin{array}{r}-57 \\
-3 \\
-67 \\
-44\end{array}$ & $\begin{array}{l}29429 \\
24749 \\
30977 \\
28562\end{array}$ & $\begin{array}{l}313 \\
400 \\
346 \\
258\end{array}$ & $\begin{array}{r}7008 \\
11704 \\
1169 \\
7026\end{array}$ & $\begin{array}{l}36750 \\
36853 \\
32492 \\
35846\end{array}$ \\
\hline $\begin{array}{r}2006 \text { Q1 } \\
\text { Q2 } \\
\text { Q3 } \\
\text { Q4 }\end{array}$ & $\begin{array}{l}36589 \\
34910 \\
34191 \\
40069\end{array}$ & $\begin{array}{l}2123 \\
1262 \\
1228 \\
1493\end{array}$ & $\begin{array}{r}116 \\
- \\
- \\
-\end{array}$ & $\begin{array}{l}- \\
- \\
- \\
-\end{array}$ & $\begin{array}{l}-121 \\
-130 \\
-126 \\
-131\end{array}$ & $\begin{array}{l}-121 \\
-130 \\
-126 \\
-131\end{array}$ & 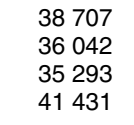 & $\begin{array}{l}28068 \\
26361 \\
28436 \\
32499\end{array}$ & $\begin{array}{r}1497 \\
-1324 \\
6037 \\
-1386\end{array}$ & $\begin{array}{r}-6 \\
26 \\
-33 \\
-29\end{array}$ & $\begin{array}{l}29559 \\
25063 \\
34440 \\
31084\end{array}$ & $\begin{array}{l}171 \\
229 \\
255 \\
214\end{array}$ & $\begin{array}{r}8977 \\
10750 \\
598 \\
10133\end{array}$ & 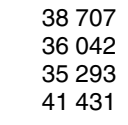 \\
\hline $\begin{array}{r}2008 \text { Q1 } \\
\text { Q2 } \\
\text { Q3 } \\
\text { Q4 }\end{array}$ & $\begin{array}{l}46055 \\
43183 \\
39509 \\
31199\end{array}$ & $\begin{array}{r}3504 \\
1121 \\
788 \\
1266\end{array}$ & $\begin{array}{l}128 \\
-50 \\
-55 \\
-73\end{array}$ & $\begin{array}{l}- \\
- \\
- \\
-\end{array}$ & $\begin{array}{l}-183 \\
-175 \\
-181 \\
-183\end{array}$ & $\begin{array}{l}-183 \\
-175 \\
-181 \\
-183\end{array}$ & $\begin{array}{l}49504 \\
44079 \\
40061 \\
32209\end{array}$ & $\begin{array}{l}31884 \\
31039 \\
31644 \\
32730\end{array}$ & $\begin{array}{r}4482 \\
575 \\
2660 \\
-6498\end{array}$ & $\begin{array}{r}-19 \\
31 \\
15 \\
-1\end{array}$ & $\begin{array}{l}36347 \\
31645 \\
34319 \\
26231\end{array}$ & $\begin{array}{l}-14 \\
238 \\
223 \\
407\end{array}$ & $\begin{array}{r}13171 \\
12196 \\
5519 \\
5571\end{array}$ & $\begin{array}{l}49504 \\
44079 \\
40061 \\
32209\end{array}$ \\
\hline $\begin{array}{r}2009 \text { Q1 } \\
\text { Q2 } \\
\text { Q3 } \\
\text { Q4 }\end{array}$ & $\begin{array}{l}37916^{\dagger} \\
39649 \\
36969 \\
41806\end{array}$ & $\begin{array}{l}5427^{\dagger} \\
1637 \\
1847 \\
2067\end{array}$ & $\begin{array}{r}194^{\dagger} \\
-18 \\
-5 \\
-10\end{array}$ & $\begin{array}{l}- \\
- \\
- \\
-\end{array}$ & $\begin{array}{l}-279^{\dagger} \\
-245 \\
-247 \\
-234\end{array}$ & $\begin{array}{l}-279^{\dagger} \\
-245 \\
-247 \\
-234\end{array}$ & $\begin{array}{l}43258^{\dagger} \\
41023 \\
38564 \\
43629\end{array}$ & $\begin{array}{l}27878^{\dagger} \\
25027 \\
25472 \\
25893\end{array}$ & $\begin{array}{r}-4351^{\dagger} \\
-5350 \\
-202 \\
-4559\end{array}$ & $\begin{array}{r}29^{\dagger} \\
-9 \\
-16 \\
15\end{array}$ & $\begin{array}{l}23556^{\dagger} \\
19668 \\
25254 \\
21349\end{array}$ & $\begin{array}{r}-69^{\dagger} \\
223 \\
81 \\
-59\end{array}$ & $\begin{array}{l}19771^{\dagger} \\
21132 \\
13229 \\
22339\end{array}$ & $\begin{array}{l}43258^{\dagger} \\
41023 \\
38564 \\
43629\end{array}$ \\
\hline \multicolumn{15}{|c|}{ Seasonally adjusted } \\
\hline $\begin{array}{r}2005 \text { Q1 } \\
\text { Q2 } \\
\text { Q3 } \\
\text { Q4 }\end{array}$ & $\begin{array}{r}\text { RPKZ } \\
33658 \\
37718 \\
32328 \\
33434\end{array}$ & $\begin{array}{r}\text { CIDZ } \\
1877 \\
1108 \\
933 \\
1222\end{array}$ & $\begin{array}{r}\text { LNZO } \\
119 \\
- \\
- \\
-\end{array}$ & $\begin{array}{r}-R N G K \\
- \\
- \\
- \\
-\end{array}$ & $\begin{array}{r}-88 \\
-124 \\
-120 \\
-124\end{array}$ & $\begin{array}{r}-R P W X \\
-88 \\
-124 \\
-120 \\
-124\end{array}$ & $\begin{array}{r}\text { RPXH } \\
35566 \\
38702 \\
33141 \\
34532\end{array}$ & $\begin{array}{l}\text { ROAW } \\
26760 \\
26214 \\
28226 \\
27779\end{array}$ & $\begin{array}{r}\text { DLQY } \\
2947 \\
1955 \\
163 \\
-156\end{array}$ & $\begin{array}{r}\text { NPOX } \\
-57 \\
-3 \\
-67 \\
-44\end{array}$ & $\begin{array}{r}\text { RQBZ } \\
29650 \\
28166 \\
28322 \\
27579\end{array}$ & $\begin{array}{r}\text { RQBW } \\
336 \\
371 \\
321 \\
289\end{array}$ & $\begin{array}{r}\text { RQBV } \\
5580 \\
10165 \\
4498 \\
6664\end{array}$ & $\begin{array}{r}\text { RPXH } \\
35566 \\
38702 \\
33141 \\
34532\end{array}$ \\
\hline $\begin{array}{r}2006 \text { Q1 } \\
\text { Q2 } \\
\text { Q3 } \\
\text { Q4 }\end{array}$ & $\begin{array}{l}37842 \\
34531 \\
34191 \\
39195\end{array}$ & $\begin{array}{l}2123 \\
1262 \\
1228 \\
1493\end{array}$ & $\begin{array}{r}116 \\
- \\
- \\
-\end{array}$ & $\begin{array}{l}- \\
- \\
- \\
-\end{array}$ & $\begin{array}{l}-121 \\
-130 \\
-126 \\
-131\end{array}$ & $\begin{array}{l}-121 \\
-130 \\
-126 \\
-131\end{array}$ & $\begin{array}{l}39960 \\
35663 \\
35293 \\
40557\end{array}$ & $\begin{array}{l}28134 \\
27839 \\
28654 \\
30737\end{array}$ & $\begin{array}{r}2328 \\
93 \\
1918 \\
485\end{array}$ & $\begin{array}{r}-6 \\
26 \\
-33 \\
-29\end{array}$ & $\begin{array}{l}30456 \\
27958 \\
30539 \\
31193\end{array}$ & $\begin{array}{l}240 \\
169 \\
208 \\
252\end{array}$ & $\begin{array}{l}9264 \\
7536 \\
4546 \\
9112\end{array}$ & $\begin{array}{l}39960 \\
35663 \\
35293 \\
40557\end{array}$ \\
\hline $\begin{array}{r}2007 \text { Q1 } \\
\text { Q2 } \\
\text { Q3 } \\
\text { Q4 }\end{array}$ & $\begin{array}{l}38242 \\
41095 \\
38331 \\
40222\end{array}$ & $\begin{array}{l}3440 \\
1098 \\
1369 \\
1704\end{array}$ & $\begin{array}{r}111 \\
- \\
- \\
-\end{array}$ & $\begin{array}{l}- \\
- \\
- \\
-\end{array}$ & $\begin{array}{l}-160 \\
-173 \\
-166 \\
-161\end{array}$ & $\begin{array}{l}-160 \\
-173 \\
-166 \\
-161\end{array}$ & $\begin{array}{l}41633 \\
42020 \\
39534 \\
41765\end{array}$ & $\begin{array}{l}31150 \\
30933 \\
33264 \\
32214\end{array}$ & $\begin{array}{r}689 \\
-103 \\
3242 \\
2816\end{array}$ & $\begin{array}{r}-9 \\
48 \\
-17 \\
-44\end{array}$ & $\begin{array}{l}31830 \\
30878 \\
36489 \\
34986\end{array}$ & $\begin{array}{r}502 \\
1321 \\
-467 \\
1133\end{array}$ & $\begin{array}{l}9301 \\
9821 \\
3512 \\
5646\end{array}$ & $\begin{array}{l}41633 \\
42020 \\
39534 \\
41765\end{array}$ \\
\hline $\begin{array}{r}2008 \text { Q1 } \\
\text { Q2 } \\
\text { Q3 } \\
\text { Q4 }\end{array}$ & $\begin{array}{l}46491 \\
42480 \\
34559 \\
36416\end{array}$ & $\begin{array}{r}3504 \\
1121 \\
788 \\
1266\end{array}$ & $\begin{array}{l}128 \\
-50 \\
-55 \\
-73\end{array}$ & $\begin{array}{l}- \\
- \\
- \\
-\end{array}$ & $\begin{array}{l}-183 \\
-175 \\
-181 \\
-183\end{array}$ & $\begin{array}{l}-183 \\
-175 \\
-181 \\
-183\end{array}$ & $\begin{array}{l}49940 \\
43376 \\
35111 \\
37426\end{array}$ & $\begin{array}{l}31974 \\
32913 \\
31965 \\
30445\end{array}$ & $\begin{array}{r}3313 \\
1622 \\
453 \\
-4169\end{array}$ & $\begin{array}{r}-19 \\
31 \\
15 \\
-1\end{array}$ & $\begin{array}{l}35268 \\
34566 \\
32433 \\
26275\end{array}$ & $\begin{array}{l}133 \\
137 \\
218 \\
366\end{array}$ & $\begin{array}{r}14539 \\
8673 \\
2460 \\
10785\end{array}$ & $\begin{array}{l}49940 \\
43376 \\
35111 \\
37426\end{array}$ \\
\hline $\begin{array}{r}2009 \text { Q1 } \\
\text { Q2 } \\
\text { Q3 } \\
\text { Q4 }\end{array}$ & $\begin{array}{l}38385^{\dagger} \\
37529 \\
34431 \\
45995\end{array}$ & $\begin{array}{l}5427^{\dagger} \\
1637 \\
1847 \\
2067\end{array}$ & $\begin{array}{r}194^{\dagger} \\
-18 \\
-5 \\
-10\end{array}$ & $\begin{array}{l}- \\
- \\
- \\
-\end{array}$ & $\begin{array}{l}-279^{\dagger} \\
-245 \\
-247 \\
-234\end{array}$ & $\begin{array}{l}-279^{\dagger} \\
-245 \\
-247 \\
-234\end{array}$ & $\begin{array}{l}43727^{\dagger} \\
38903 \\
36026 \\
47818\end{array}$ & $\begin{array}{l}27969^{\dagger} \\
26571 \\
25349 \\
24381\end{array}$ & $\begin{array}{l}-4196^{\dagger} \\
-2895 \\
-4882 \\
-2489\end{array}$ & $\begin{array}{r}29^{\dagger} \\
-9 \\
-16 \\
15\end{array}$ & $\begin{array}{l}23802^{\dagger} \\
23667 \\
20451 \\
21907\end{array}$ & $\begin{array}{r}102^{\dagger} \\
126 \\
77 \\
-129\end{array}$ & $\begin{array}{l}19823^{\dagger} \\
15110 \\
15498 \\
26040\end{array}$ & $\begin{array}{l}43727^{\dagger} \\
38903 \\
36026 \\
47818\end{array}$ \\
\hline
\end{tabular}


ALLOCATION OF PRIMARY INCOME ACCOUNT

\begin{tabular}{|c|c|c|c|c|c|c|c|c|c|c|c|c|c|c|c|c|c|}
\hline & \multicolumn{8}{|c|}{ RESOURCES } & \multicolumn{9}{|c|}{ USES } \\
\hline & \multicolumn{7}{|c|}{ D.4 Property income, received } & & \multicolumn{6}{|c|}{ D.4 Property income,paid } & \multirow[b]{2}{*}{$\begin{array}{r}\text { Balance } \\
\text { of } \\
\text { gross } \\
\text { primary } \\
\text { income }\end{array}$} & \multirow[b]{2}{*}{$\begin{array}{l}\text { Total } \\
\text { uses }\end{array}$} & \\
\hline & $\begin{array}{r}\text { Gross } \\
\text { Operat- } \\
\text { ing } \\
\text { surplus }\end{array}$ & $\begin{array}{r}\text { Intere- } \\
\text { st }\end{array}$ & $\begin{array}{r}\text { Distri- } \\
\text { buted } \\
\text { income } \\
\text { of } \\
\text { corps. }\end{array}$ & $\begin{array}{r}\text { Reinve- } \\
\text { sted } \\
\text { earnin- } \\
\text { gs on } \\
\text { direct } \\
\text { foreign } \\
\text { invest- } \\
\text { ment }\end{array}$ & $\begin{array}{r}\text { Proper- } \\
\text { ty inc } \\
\text { to } \\
\text { insura- } \\
\text { nce } \\
\text { holders }\end{array}$ & Rent & Total & $\begin{array}{r}\text { Total } \\
\text { resour- } \\
\text { ces }\end{array}$ & $\begin{array}{r}\text { Intere- } \\
\text { st }\end{array}$ & $\begin{array}{r}\text { Distri- } \\
\text { buted } \\
\text { income } \\
\text { of } \\
\text { corps }\end{array}$ & $\begin{array}{r}\text { Reinve- } \\
\text { sted } \\
\text { earnin- } \\
\text { gs on } \\
\text { direct } \\
\text { foreign } \\
\text { invest- } \\
\text { ment }\end{array}$ & $\begin{array}{r}\text { Proper- } \\
\text { ty inc } \\
\text { to } \\
\text { insura- } \\
\text { nce } \\
\text { holders }\end{array}$ & Rent & Total & & & $\begin{array}{r}\text { Share } \\
\text { of } \\
\text { gross } \\
\text { nation- } \\
\text { al } \\
\text { income }\end{array}$ \\
\hline & B.2g & D.41 & D.42 & D.43 & D.44 & D.45 & D. 4 & $-\mathrm{P} 119$ & TR & D.41 & D.42 & D.43 & D.44 & D.45 & D.4 & B.5G & \\
\hline & NQNV & NHCK & NHCL & NHEM & NHDG & $\mathrm{NHDH}$ & NHDF & NQNW & $\mathrm{NHCM}$ & $\mathrm{NHCN}$ & NHEO & NSCU & NHDK & NHDI & NQNY & NQNW & RVG \\
\hline 2001 & 12965 & 198445 & 49801 & 4223 & 37 & 29 & 252535 & 265500 & 170669 & 43922 & -2691 & 54795 & - & 266695 & -1195 & 265500 & -0 \\
\hline 2002 & 27125 & 162001 & 51990 & 5278 & 34 & 30 & 219333 & 246458 & 133981 & 37207 & 2033 & 53652 & - & 226873 & 19585 & 246458 & \\
\hline 2003 & 33218 & 160855 & 56472 & 8897 & 44 & 30 & 226298 & 259516 & 129917 & 43137 & 3474 & 56715 & - & 233243 & 26273 & 259516 & \\
\hline 2004 & 32879 & 195443 & 65273 & 8208 & 39 & 31 & 268994 & 301873 & 159828 & 52167 & 2233 & 56150 & - & 270378 & 31495 & 301873 & \\
\hline 2005 & 33135 & 246249 & 70120 & 10201 & 66 & 31 & 326667 & 359802 & 212659 & 51797 & 5518 & 65805 & - & 335779 & 24023 & 359802 & \\
\hline 2006 & 38847 & 316876 & 82884 & 11153 & 59 & 31 & 411003 & 449850 & 284160 & 63327 & 6743 & 68321 & & 422551 & 27299 & 449850 & \\
\hline 2007 & 46377 & 414451 & 92358 & 9252 & 32 & 32 & 516125 & 562502 & 373272 & 62128 & 8957 & 72820 & - & 517177 & 45325 & 562502 & \\
\hline 2008 & 63695 & 392487 & 78593 & 682 & 39 & 32 & 471833 & 535528 & 351861 & 33409 & 816 & 76424 & - & 462510 & 73018 & 535528 & \\
\hline 2009 & 66938 & 169479 & 63870 & 638 & 33 & 31 & 234051 & 300989 & 147882 & 31938 & 10925 & 70258 & - & 261003 & 39986 & 300989 & \\
\hline
\end{tabular}

Not Seasonally adjusted

\begin{tabular}{|c|c|c|c|c|}
\hline 2005 Q1 & 7949 & 57307 & 14004 & 2672 \\
\hline Q2 & 9301 & 60631 & 22965 & 2172 \\
\hline Q3 & 7230 & 61812 & 14752 & 2191 \\
\hline Q4 & 8655 & 66499 & 18399 & 3166 \\
\hline 2006 Q1 & 7951 & 71769 & 14064 & 3361 \\
\hline Q2 & 10748 & 76733 & 29722 & 2790 \\
\hline Q3 & 10349 & 81233 & 17502 & 2775 \\
\hline Q4 & 9799 & 87141 & 21596 & 2227 \\
\hline 2007 Q1 & 10990 & 95541 & 16741 & 2462 \\
\hline Q2 & 10442 & 103429 & 34913 & 2011 \\
\hline Q3 & 11808 & 108139 & 19174 & 2796 \\
\hline Q4 & 13137 & 107342 & 21530 & 1983 \\
\hline 2008 Q1 & 13486 & 102320 & 16041 & 1477 \\
\hline Q2 & 15246 & 98547 & 27128 & 1582 \\
\hline Q3 & 14821 & 100480 & 17547 & 3405 \\
\hline Q4 & 20142 & 91140 & 17877 & -5782 \\
\hline 09 Q1 & $16222^{\dagger}$ & $54741^{\dagger}$ & $12227^{\dagger}$ & $2029^{\dagger}$ \\
\hline Q2 & 16046 & 45150 & 17261 & 1031 \\
\hline Q3 & 17556 & 34500 & 14753 & 613 \\
\hline Q4 & 17114 & 35088 & 19629 & -3035 \\
\hline
\end{tabular}

\begin{tabular}{|c|c|c|c|c|c|c|}
\hline & 74003 & 81952 & 48349 & 9002 & 1395 & 15933 \\
\hline & 85794 & 95095 & 52674 & 17734 & 1028 & 17296 \\
\hline & 78782 & 86012 & 53998 & 8071 & 1485 & 17161 \\
\hline & 88088 & 96743 & 57638 & 16990 & 1610 & 15415 \\
\hline & 89211 & & 63362 & & 1745 & 16729 \\
\hline & 1092 & 120 & & 23 & & 17671 \\
\hline & & & & & & \\
\hline & & & & 182 & & \\
\hline & 7 & & & & & \\
\hline & & & & & & 255 \\
\hline & & & & 13 & 66 & 641 \\
\hline & 130 & & & 7774 & 1829 & 637 \\
\hline & 33 & & & & 10 & 18801 \\
\hline & 127276 & 142 & 20 & 15146 & 2526 & 20697 \\
\hline & 121448 & 1362 & & 9375 & -1 & 416 \\
\hline & 1032 & 123398 & & 5660 & & 18510 \\
\hline & $69013^{\dagger}$ & $85235^{\dagger}$ & $50875^{\dagger}$ & $2654^{\dagger}$ & 2479 & 15191 \\
\hline & & & & 11511 & & 19498 \\
\hline & 4988 & & & & & 20224 \\
\hline & 51698 & 68812 & 27639 & 10781 & 3705 & 15345 \\
\hline
\end{tabular}

$\begin{array}{lrrr}- & 74679 & 7273 & 81952 \\ - & 88732 & 6363 & 95095 \\ - & 80715 & 5297 & 86012 \\ - & 91653 & 5090 & 96743 \\ & & \\ - & 91470 & 5692 & 97162 \\ - & 112397 & 7619 & 120016 \\ - & 103419 & 8456 & 111875 \\ - & 115265 & 5532 & 120797 \\ & \\ - & 116361 & 9386 & 125747 \\ - & 143941 & 6869 & 150810 \\ - & 131818 & 10112 & 141930 \\ - & 125057 & 18958 & 144015 \\ & & \\ - & 113873 & 19466 & 133339 \\ - & 127589 & 14933 & 142522 \\ - & 116264 & 20005 & 136269 \\ - & 104784 & 18614 & 123398 \\ - & 711999^{\dagger} & 14036^{\dagger} & 85235^{\dagger} \\ - & 72387 & 7115 & 79502 \\ - & 59947 & 7493 & 67440 \\ - & 57470 & 11342 & 68812\end{array}$

2.3

2.0

Seasonally adjusted

\begin{tabular}{|c|c|c|c|c|c|c|c|c|c|c|c|c|c|c|c|c|c|}
\hline & $\mathrm{NHCZ}$ & ROWJ & ROWK & ROWL & ROWM & ROCO & ROWI & ROWN & ROWQ & ROWR & ROWS & RNRD & $\mathrm{ROBH}$ & ROWP & ROWO & ROWN & NRJI \\
\hline 2005 Q1 & 8518 & 56947 & 19031 & 2672 & 12 & 8 & 78670 & 87188 & 48986 & 13922 & 1354 & 15972 & 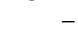 & 80234 & 6954 & 87188 & 2.2 \\
\hline Q2 & 9005 & 60708 & 15443 & 2172 & 18 & 8 & 78349 & 87354 & 51834 & 11006 & 1017 & 16579 & - & 80436 & 6918 & 87354 & 2.2 \\
\hline Q3 & 7162 & 62279 & 17463 & 2191 & 19 & 8 & 81960 & 89122 & 54063 & 12109 & 1396 & 16983 & - & 84551 & 4571 & 89122 & 1.4 \\
\hline Q4 & 8450 & 66315 & 18183 & 3166 & 17 & 7 & 87688 & 96138 & 57776 & 14760 & 1751 & 16271 & - & 90558 & 5580 & 96138 & 1.7 \\
\hline 2006 Q1 & 8707 & 71720 & 19044 & 3361 & 9 & 8 & 94142 & 102849 & 64361 & 15859 & 1729 & 16883 & - & 98832 & 4017 & 102849 & 1.2 \\
\hline Q2 & 10616 & 77491 & 20903 & 2790 & 15 & 8 & 101207 & 111823 & 69038 & 15359 & 2036 & 16946 & & 103379 & 8444 & 111823 & 2.6 \\
\hline Q3 & 10017 & 81706 & 20826 & 2775 & 8 & 8 & 105323 & 115340 & 72921 & 15254 & 1083 & 17157 & - & 106415 & 8925 & 115340 & 2.7 \\
\hline Q4 & 9507 & 85959 & 22111 & 2227 & 27 & 7 & 110331 & 119838 & 77840 & 16855 & 1895 & 17335 & - & 113925 & 5913 & 119838 & 1.7 \\
\hline 2007 Q1 & 1910 & 96969 & 22645 & 2462 & 5 & 8 & 122089 & 133999 & 87188 & 17750 & 2912 & 17560 & - & 125410 & 8589 & 133999 & 2.5 \\
\hline Q2 & 10384 & 105996 & 25026 & 2011 & 7 & 8 & 133048 & 143432 & 95726 & 20302 & 1972 & 17420 & & 135420 & 8012 & 143432 & 2.3 \\
\hline Q3 & 11272 & 107606 & 22662 & 2796 & 5 & 8 & 133077 & 144349 & 96523 & 16562 & 2076 & 18406 & - & 133567 & 10782 & 144349 & 3.0 \\
\hline Q4 & 12811 & 103880 & 22025 & 1983 & 15 & 8 & 127911 & 140722 & 93835 & 7514 & 1997 & 19434 & - & 122780 & 17942 & 140722 & 4.9 \\
\hline 2008 Q1 & 14355 & 104563 & 20911 & 1477 & 7 & 8 & 126966 & 141321 & 93191 & 9839 & 1018 & 19278 & - & 123326 & 17995 & 141321 & 4.8 \\
\hline Q2 & 15107 & 101350 & 18438 & 1582 & 11 & 8 & 121389 & 136496 & 91477 & 7103 & 2540 & 19733 & - & 120853 & 15643 & 136496 & 4.2 \\
\hline Q3 & 14424 & 100394 & 20722 & 3405 & 8 & 8 & 124537 & 138961 & 89176 & 9839 & -2061 & 18152 & - & 115106 & 23855 & 138961 & 6.5 \\
\hline Q4 & 19809 & 86180 & 18522 & -5782 & 13 & 8 & 98941 & 118750 & 78017 & 6628 & -681 & 19261 & - & 103225 & 15525 & 118750 & 4.3 \\
\hline Q2 & 16281 & 47534 & 11152 & 1031 & 6 & 8 & 59731 & 76012 & 41019 & 5325 & 1575 & 18377 & - & 66296 & 9716 & 76012 & 2.8 \\
\hline Q3 & 16820 & 34042 & 17072 & 613 & 10 & 8 & 51745 & 68565 & 29213 & 7269 & 2918 & 19516 & - & 58916 & 9649 & 68565 & 2.7 \\
\hline Q4 & 16725 & 31849 & 18801 & -3035 & 9 & 7 & 47631 & 64356 & 25156 & 10242 & 3816 & 16447 & - & 55661 & 8695 & 64356 & 2.4 \\
\hline
\end{tabular}


SECONDARY DISTRIBUTION OF INCOME ACCOUNT

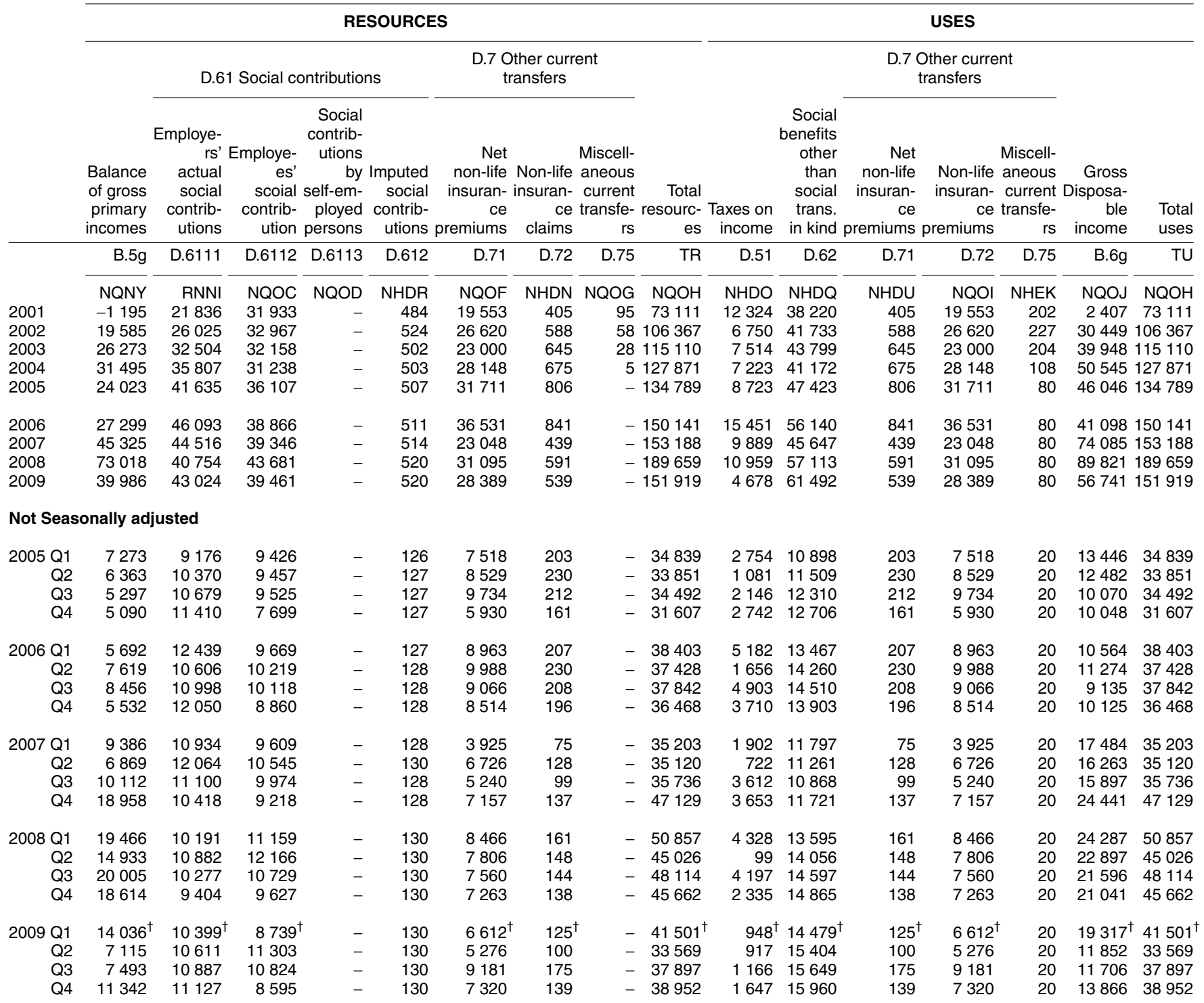

\section{Seasonally adjusted}

$\begin{array}{rrrrr}2005 \text { Q1 } & \text { ROW } & & \text { RNNP } & \text { RNN } \\ \text { Q2 } & 6918 & 9176 & 9088 & - \\ \text { Q3 } & 4571 & 10679 & 9922 & - \\ \text { Q4 } & 5580 & 11410 & 8798 & - \\ & & & & \\ 2006 \text { Q1 } & 4017 & 12439 & 9402 & - \\ \text { Q2 } & 8444 & 10606 & 9568 & - \\ \text { Q3 } & 8925 & 10998 & 9906 & - \\ \text { Q4 } & 5913 & 12050 & 9990 & - \\ & & & & - \\ 2007 \text { Q1 } & 8589 & 10934 & 9491 & - \\ \text { Q2 } & 8012 & 12064 & 9731 & - \\ \text { Q3 } & 10782 & 11100 & 9810 & - \\ \text { Q4 } & 17942 & 10418 & 10314 & - \\ & & & & - \\ 2008 \text { Q1 } & 17995 & 10191 & 11187 & - \\ \text { Q2 } & 15643 & 10882 & 11064 & - \\ \text { Q3 } & 23855 & 10277 & 10690 & - \\ \text { Q4 } & 15525 & 9404 & 10740 & - \\ & & & & - \\ 2009 \text { Q1 } & 11926^{\dagger} & 10399^{\dagger} & 8919^{\dagger} & - \\ \text { Q2 } & 9716 & 10611 & 10183 & - \\ \text { Q3 } & 9649 & 10887 & 10475 & - \\ \text { Q4 } & 8695 & 11127 & 9884 & -\end{array}$

$\begin{array}{llr} & \text { RPEE } & \text { RPEF } \\ 126 & 7489 & 202 \\ 127 & 8230 & 222 \\ 127 & 9641 & 212 \\ 127 & 6351 & 170 \\ & & \\ 127 & 9089 & 208 \\ 128 & 9485 & 221 \\ 128 & 9123 & 209 \\ 128 & 8834 & 203 \\ & & \\ 128 & 4006 & 76 \\ 130 & 6364 & 123 \\ 128 & 5400 & 101 \\ 128 & 7278 & 139 \\ & & \\ 130 & 7973 & 152 \\ 130 & 7926 & 151 \\ 130 & 7740 & 146 \\ 130 & 7456 & 142 \\ & & \\ 130 & 6419^{\dagger} & 121 \\ 130 & 5505 & 108 \\ 130 & 9173 & 173 \\ 130 & 7292 & 137\end{array}$

\begin{tabular}{|c|c|c|c|c|}
\hline EF & RPEG & RPEH & RPEK & NROD \\
\hline 202 & - & 33035 & 2554 & 11006 \\
\hline 22 & - & 34789 & 2338 & 11595 \\
\hline & - & 34529 & 1562 & 12284 \\
\hline & - & 32436 & 2269 & 12538 \\
\hline & - & 35282 & 4873 & 13662 \\
\hline & - & 38452 & 3187 & 14365 \\
\hline 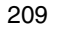 & - & 39289 & 4230 & 14449 \\
\hline & - & 37118 & 3161 & 13664 \\
\hline & - & 33224 & 1610 & 12001 \\
\hline & - & 36424 & 2316 & 11350 \\
\hline & - & 37321 & 2886 & 10804 \\
\hline & - & 46219 & 3077 & 11492 \\
\hline & - & 47628 & 4138 & 13574 \\
\hline & - & 45796 & 1668 & 14139 \\
\hline & - & 52838 & 3329 & 14585 \\
\hline & - & 43397 & 1824 & 14815 \\
\hline 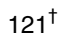 & - & $37914^{\dagger}$ & $804^{\dagger}$ & $14658^{\dagger}$ \\
\hline & - & 36253 & 2437 & 15261 \\
\hline & - & 40487 & 241 & 15695 \\
\hline & - & 37265 & 1196 & 15878 \\
\hline
\end{tabular}

RPEP $\begin{array}{lllll}222 & 8230 & 20 & 12384 & 34789\end{array}$ $\begin{array}{lllll}170 & 6351 & 20 & 11088 & 32436\end{array}$

$\begin{array}{lllll}208 & 9089 & 20 & 7430 & 35282\end{array}$ $\begin{array}{llllll}221 & 9485 & 20 & 11174 & 38452\end{array}$ $\begin{array}{lllll}209 & 9123 & 20 & 11258 & 39289\end{array}$ $\begin{array}{lllll}203 & 8834 & 20 & 11236 & 37118\end{array}$

$\begin{array}{lllll}76 & 4006 & 20 & 15511 & 33224\end{array}$ $\begin{array}{llllll}123 & 6364 & 20 & 16251 & 36424\end{array}$ $\begin{array}{llllll}101 & 5400 & 20 & 18110 & 37321\end{array}$ $\begin{array}{lllll}139 & 7278 & 20 & 24213 & 46219\end{array}$

$\begin{array}{lllll}152 & 7973 & 20 & 21771 & 47628\end{array}$ $\begin{array}{lllll}151 & 7926 & 20 & 21892 & 45796\end{array}$ $\begin{array}{lllll}146 & 7740 & 20 & 27018 & 52838\end{array}$ $\begin{array}{lllll}142 & 7456 & 20 & 19140 & 43397\end{array}$

$121^{\dagger} \quad 6419^{\dagger} \quad 20 \quad 15892^{\dagger} 37914^{\dagger}$ $\begin{array}{lllll}108 & 5505 & 20 & 12922 & 36253\end{array}$ $\begin{array}{lllll}173 & 9173 & 20 & 15185 & 40487\end{array}$ $\begin{array}{lllll}137 & 7292 & 20 & 12742 & 37265\end{array}$ 


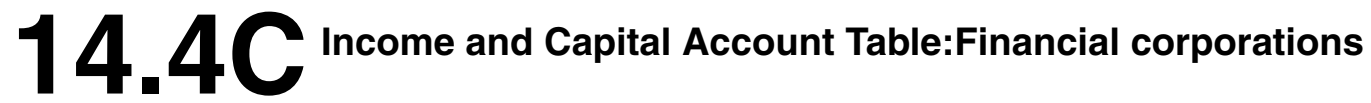

\begin{tabular}{|c|c|c|c|c|}
\hline & & & & $£$ million \\
\hline & \multicolumn{4}{|c|}{ USE OF DISPOSABLE INCOME ACCOUNT } \\
\hline & \multirow[b]{2}{*}{$\begin{array}{l}\text { Total resources, gross } \\
\text { disposable income }\end{array}$} & \multicolumn{3}{|c|}{ USES } \\
\hline & & $\begin{array}{r}\text { Adjustment for the change in } \\
\text { net equity of } h / \text { holds in } \\
\text { pens.fund }\end{array}$ & Gross Saving & Total uses \\
\hline & B.6g & D.8 & B.8g & $\overline{T U}$ \\
\hline & NQOJ & NQOK & NQOL & NQOJ \\
\hline 2001 & 2407 & 16033 & -13626 & 2407 \\
\hline 2002 & 30449 & 17783 & 12666 & 30449 \\
\hline 2003 & 39948 & 21365 & 18583 & 39948 \\
\hline 2004 & 50545 & 26375 & 24170 & 50545 \\
\hline 2005 & 46046 & 30826 & 15220 & 46046 \\
\hline 2006 & 41098 & 29330 & 11768 & 41098 \\
\hline 2007 & 74085 & 38729 & 35356 & 74085 \\
\hline 2008 & 89821 & 27842 & 61979 & 89821 \\
\hline 2009 & 56741 & 21513 & 35228 & 56741 \\
\hline \multicolumn{5}{|c|}{ Not Seasonally adjusted } \\
\hline 2005 Q1 & 13446 & 8950 & 4496 & 13446 \\
\hline Q2 & 12482 & 7220 & 5262 & 12482 \\
\hline Q3 & 10070 & 6937 & 3133 & 10070 \\
\hline Q4 & 10048 & 7719 & 2329 & 10048 \\
\hline 2006 Q1 & 10564 & 10074 & 490 & 10564 \\
\hline Q2 & 11274 & 5331 & 5943 & 11274 \\
\hline Q3 & 9135 & 5602 & 3533 & 9135 \\
\hline Q4 & 10125 & 8323 & 1802 & 10125 \\
\hline 2007 Q1 & 17484 & 10020 & 7464 & 17484 \\
\hline Q2 & 16263 & 10136 & 6127 & 16263 \\
\hline Q3 & 15897 & 9417 & 6480 & 15897 \\
\hline Q4 & 24441 & 9156 & 15285 & 24441 \\
\hline 2008 Q1 & 24287 & 9169 & 15118 & 24287 \\
\hline Q2 & 22897 & 8083 & 14814 & 22897 \\
\hline Q3 & 21596 & 5808 & 15788 & 21596 \\
\hline Q4 & 21041 & 4782 & 16259 & 21041 \\
\hline 2009 Q1 & $19317^{\dagger}$ & $6249^{\dagger}$ & $13068^{\dagger}$ & $19317^{\dagger}$ \\
\hline Q2 & 11852 & 5674 & 6178 & 11852 \\
\hline Q3 & 11706 & 5399 & 6307 & 11706 \\
\hline Q4 & 13866 & 4191 & 9675 & 13866 \\
\hline \multicolumn{5}{|c|}{ Seasonally adjusted } \\
\hline & RPEI & RPPT & RPPS & RPEI \\
\hline 2005 Q1 & 11764 & 7384 & 4380 & 11764 \\
\hline Q2 & 12384 & 7824 & 4560 & 12384 \\
\hline Q3 & 10810 & 7821 & 2989 & 10810 \\
\hline Q4 & 11088 & 7797 & 3291 & 11088 \\
\hline 2006 Q1 & 7430 & 8306 & -876 & 7430 \\
\hline Q2 & 11174 & 5937 & 5237 & 11174 \\
\hline Q3 & 11258 & 6583 & 4675 & 11258 \\
\hline Q4 & 11236 & 8504 & 2732 & 11236 \\
\hline 2007 Q1 & 15511 & 8552 & 6959 & 15511 \\
\hline Q2 & 16251 & 10575 & 5676 & 16251 \\
\hline Q3 & 18110 & 10234 & 7876 & 18110 \\
\hline Q4 & 24213 & 9368 & 14845 & 24213 \\
\hline 2008 Q1 & 21771 & 7934 & 13837 & 21771 \\
\hline Q2 & 21892 & 7937 & 13955 & 21892 \\
\hline Q3 & 27018 & 6512 & 20506 & 27018 \\
\hline Q4 & 19140 & 5459 & 13681 & 19140 \\
\hline 2009 Q1 & $15892^{\dagger}$ & $4790^{\dagger}$ & $11102^{\dagger}$ & $15892^{\dagger}$ \\
\hline Q2 & 12922 & 5663 & 7259 & 12922 \\
\hline Q3 & 15185 & 5797 & 9388 & 15185 \\
\hline Q4 & 12742 & 5263 & 7479 & 12742 \\
\hline
\end{tabular}

Source: National Statistics 


$$
\text { ACCUMULATION ACCOUNTS }
$$

CAPITAL ACCOUNT

CHANGE IN NET WORTH DUE TO SAVING AND CAPITAL TRANSFERS

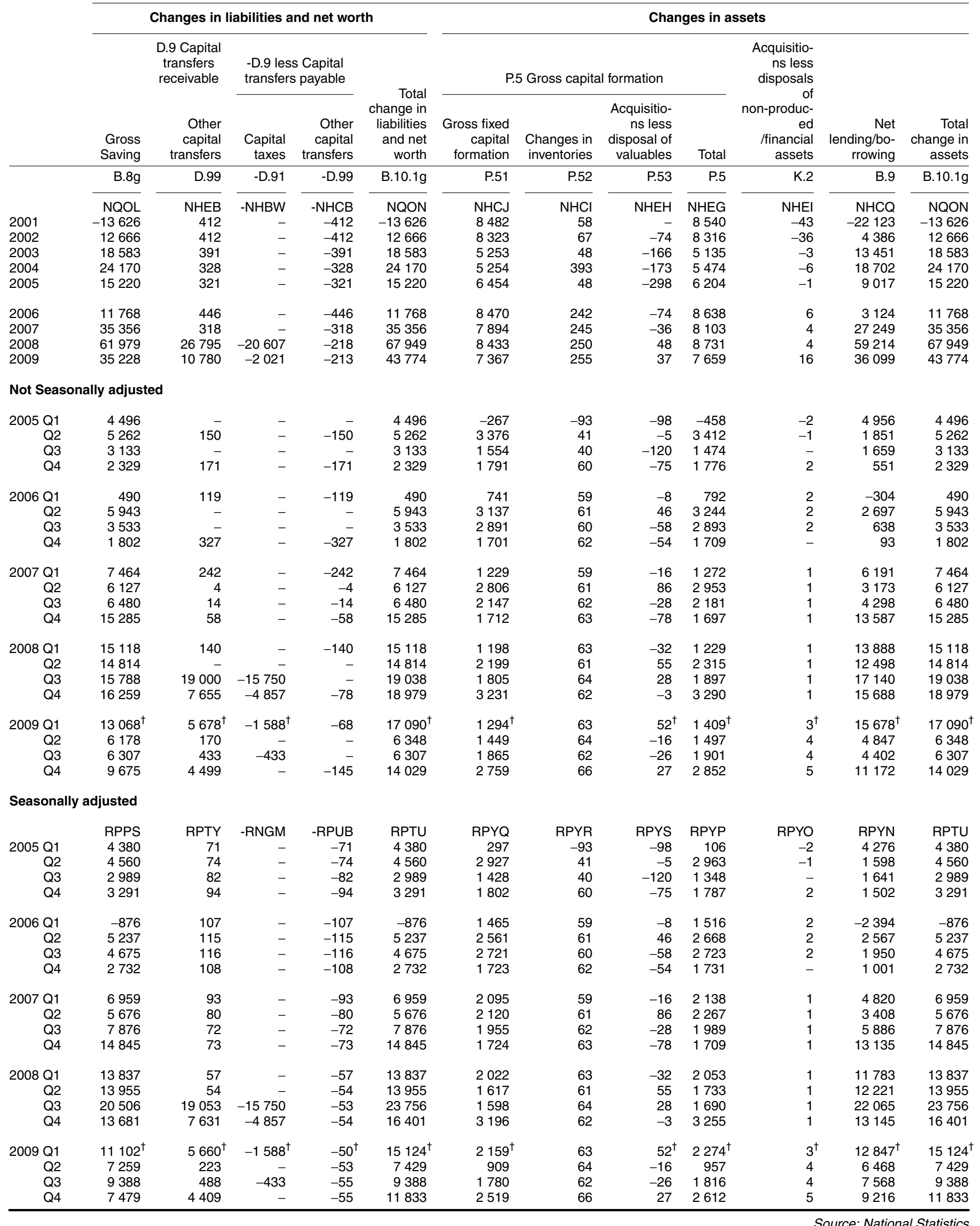




\begin{tabular}{|c|c|c|c|c|c|c|c|c|c|c|c|c|}
\hline & \multicolumn{12}{|c|}{ ALLOCATION OF PRIMARY INCOME ACCOUNT } \\
\hline & \multicolumn{12}{|c|}{ RESOURCES } \\
\hline & \multicolumn{5}{|c|}{ D.2 Taxes on production and imports, received } & \multicolumn{2}{|c|}{ less subsidies paid } & \multicolumn{4}{|c|}{ D.4 Property Income } & \multirow[b]{2}{*}{$\begin{array}{r}\text { Total } \\
\text { resources } \\
\end{array}$} \\
\hline & $\begin{array}{r}\text { Gross } \\
\text { Operating } \\
\text { surplus }\end{array}$ & $\begin{array}{r}\text { Value added } \\
\text { tax(VAT) }\end{array}$ & $\begin{array}{r}\text { Taxes on } \\
\text { imports } \\
\text { excluding } \\
\text { VAT }\end{array}$ & $\begin{array}{r}\text { Taxes on } \\
\text { products } \\
\text { excluding } \\
\text { VAT and } \\
\text { import } \\
\text { duties }\end{array}$ & $\begin{array}{r}\text { Other taxes } \\
\text { on } \\
\text { production }\end{array}$ & $\begin{array}{r}\text { Subsidies } \\
\text { on products }\end{array}$ & $\begin{array}{r}\text { Other } \\
\text { subsidies } \\
\text { on } \\
\text { production }\end{array}$ & Interest & $\begin{array}{c}\text { Distributed } \\
\text { income of } \\
\text { corps. }\end{array}$ & $\begin{array}{r}\text { Rent from } \\
\text { sectors } \\
\text { other than } \\
\text { general } \\
\text { government }\end{array}$ & $\begin{array}{r}\text { Total } \\
\text { property } \\
\text { income }\end{array}$ & \\
\hline & B.2g & D.211 & D.212 & D.214 & D.29 & -D.31 & -D.39 & D.41 & D.42 & D.45 & D.4 & $\mathrm{TR}$ \\
\hline & NMXV & NZGF & NMBU & NMBV & NMYD & -NMYF & -NMCC & NMYL & NMYM & NMYR & NMYU & NMYV \\
\hline 2001 & 9796 & 63522 & - & 50745 & 17565 & -3953 & -653 & 7359 & 4710 & 1919 & 14012 & 151025 \\
\hline 2002 & 10289 & 68251 & - & 52001 & 18113 & -4672 & -937 & 6683 & 3290 & 1901 & 11892 & 154920 \\
\hline 2003 & 10807 & 74595 & - & 52858 & 18517 & -5311 & -1416 & 7131 & 3027 & 1565 & 11742 & 161774 \\
\hline 2004 & 11312 & 79755 & - & 56137 & 18853 & -5121 & -1323 & 6838 & 2794 & 1182 & 10833 & 170207 \\
\hline 2005 & 11927 & 81426 & - & 56906 & 19706 & -5182 & -1383 & 6471 & 2900 & 1229 & 10627 & 172961 \\
\hline 2007 & 13171 & 89698 & - & 64309 & 21543 & -5591 & -1961 & 8109 & 3153 & 1233 & 12515 & 192175 \\
\hline 2008 & 13859 & 89682 & - & 60666 & 23043 & -5658 & -1673 & 8780 & 3149 & 1161 & 13114 & 191405 \\
\hline \multicolumn{13}{|c|}{ Not Seasonally adjusted } \\
\hline 2005 Q1 & 2924 & 19526 & - & 13081 & 4714 & -1382 & -320 & 1826 & 703 & 300 & 2834 & 40802 \\
\hline Q2 & 2960 & 20146 & - & 14131 & 4991 & -1140 & -352 & 1428 & 909 & 320 & 2664 & 43377 \\
\hline Q3 & 3000 & 20736 & - & 14735 & 5026 & -1327 & -353 & 1576 & 635 & 304 & 2523 & 44147 \\
\hline Q4 & 3043 & 21018 & - & 14959 & 4975 & -1333 & -358 & 1641 & 653 & 305 & 2606 & 44635 \\
\hline 2006 Q1 & 3093 & 19605 & - & 14140 & 4988 & -1391 & -361 & 2003 & 579 & 295 & 2881 & 41921 \\
\hline Q2 & 3137 & 21299 & - & 15037 & 5293 & -1393 & -342 & 1637 & 753 & 321 & 2717 & 45724 \\
\hline Q3 & 3186 & 22155 & - & 15334 & 5288 & -1762 & -343 & 1556 & 575 & 304 & 2438 & 45995 \\
\hline Q4 & 3231 & 22532 & - & 16029 & 5262 & -1427 & -386 & 1941 & 663 & 306 & 2922 & 47861 \\
\hline 2007 Q1 & 3250 & 21753 & - & 14868 & 5276 & -1162 & -406 & 1998 & 652 & 298 & 2951 & 45535 \\
\hline Q2 & 3277 & 22369 & - & 16307 & 5395 & -1442 & -511 & 1799 & 947 & 345 & 3095 & 48467 \\
\hline Q3 & 3304 & 22848 & - & 16461 & 5459 & -1456 & -676 & 1880 & 739 & 299 & 2922 & 48655 \\
\hline Q4 & 3340 & 22728 & - & 16673 & 5413 & -1531 & -368 & 2432 & 815 & 291 & 3547 & 49518 \\
\hline Q3 & 3495 & 22519 & - & 14982 & 5812 & -1486 & -336 & 2381 & 693 & 319 & 3398 & 48187 \\
\hline Q4 & 3547 & 21003 & - & 15157 & 5964 & -1326 & -339 & 2084 & 677 & 289 & 3058 & 46767 \\
\hline 2009 Q1 & $3593^{\dagger}$ & $17430^{\dagger}$ & - & $13272^{\dagger}$ & $6024^{\dagger}$ & $-1212^{\dagger}$ & $-346^{\dagger}$ & $2169^{\dagger}$ & $428^{\dagger}$ & $297^{\dagger}$ & $2899^{\dagger}$ & $40521^{\dagger}$ \\
\hline Q2 & 3652 & 18962 & - & 15129 & 6087 & -1596 & -487 & 1580 & 754 & 292 & 2630 & 44353 \\
\hline Q3 & 3708 & 19959 & - & 15600 & 6099 & -1714 & -490 & 1374 & 305 & 291 & 1976 & 44912 \\
\hline Q4 & 3748 & 20562 & - & 16348 & 6038 & -1766 & -495 & 1392 & 481 & 292 & 2170 & 46421 \\
\hline \multicolumn{13}{|c|}{ Seasonally adjusted } \\
\hline & NTAR & RNDC & & RNDL & NTAI & -ROXF & -RNES & ROXJ & ROXM & $\mathrm{ROXQ}$ & ROXG & ROXT \\
\hline 2005 Q1 & 2924 & 20190 & - & 13990 & 4804 & -1282 & -320 & 1832 & 712 & 300 & 2849 & 42580 \\
\hline Q2 & 2960 & 20254 & - & 14123 & 4891 & -1215 & -352 & 1441 & 915 & 320 & 2683 & 43321 \\
\hline Q3 & 3000 & 20514 & - & 14479 & 4993 & -1359 & -353 & 1566 & 639 & 304 & 2517 & 43598 \\
\hline Q4 & 3043 & 20468 & - & 14314 & 5018 & -1326 & -358 & 1632 & 634 & 305 & 2578 & 43462 \\
\hline 2006 Q1 & 3093 & 20355 & - & 15126 & 5128 & -1423 & -361 & 2010 & 588 & 295 & 2897 & 43781 \\
\hline Q2 & 3137 & 21411 & - & 15002 & 5203 & -1505 & -342 & 1656 & 759 & 321 & 2742 & 45624 \\
\hline Q3 & 3186 & 21883 & - & 15032 & 5243 & -1562 & -343 & 1542 & 580 & 304 & 2429 & 45567 \\
\hline Q4 & 3231 & 21942 & - & 15380 & 5257 & -1483 & -386 & 1929 & 643 & 306 & 2890 & 46529 \\
\hline 2007 Q1 & 3250 & 22603 & - & 15865 & 5333 & -1493 & -406 & 2010 & 661 & 298 & 2972 & 47129 \\
\hline Q2 & 3277 & 22461 & - & 16266 & 5339 & -1376 & -511 & 1831 & 954 & 345 & 3134 & 48567 \\
\hline Q3 & 3304 & 22506 & - & 16136 & 5429 & -1374 & -676 & 1857 & 746 & 299 & 2906 & 48024 \\
\hline Q4 & 3340 & 22128 & - & 16042 & 5442 & -1348 & -368 & 2411 & 792 & 291 & 3503 & 48455 \\
\hline 2008 Q1 & 3384 & 22999 & - & 16026 & 5598 & -1336 & -370 & 2329 & 811 & 291 & 3435 & 48625 \\
\hline Q2 & 3433 & 24107 & - & 15379 & 5681 & -1401 & -628 & 2046 & 980 & 262 & 3295 & 49843 \\
\hline Q3 & 3495 & 21935 & - & 14727 & 5754 & -1470 & -336 & 2351 & 703 & 319 & 3378 & 47286 \\
\hline Q4 & 3547 & 20641 & - & 14534 & 6010 & -1451 & -339 & 2054 & 655 & 289 & 3006 & 45651 \\
\hline 2009 Q1 & $3593^{\dagger}$ & $18502^{\dagger}$ & - & $14139^{\dagger}$ & $6046^{\dagger}$ & $-1388^{\dagger}$ & $-346^{\dagger}$ & $2173^{\dagger}$ & $434^{\dagger}$ & $297^{\dagger}$ & $2909^{\dagger}$ & $42316^{\dagger}$ \\
\hline Q2 & 3652 & 18902 & - & 14993 & 6058 & -1647 & -487 & 1593 & 761 & 292 & 2650 & 44097 \\
\hline Q3 & 3708 & 19410 & - & 15458 & 6074 & -1724 & -490 & 1366 & 314 & 291 & 1977 & 44187 \\
\hline Q4 & 3748 & 20099 & - & 15759 & 6070 & -1529 & -495 & 1383 & 459 & 292 & 2139 & 45607 \\
\hline
\end{tabular}




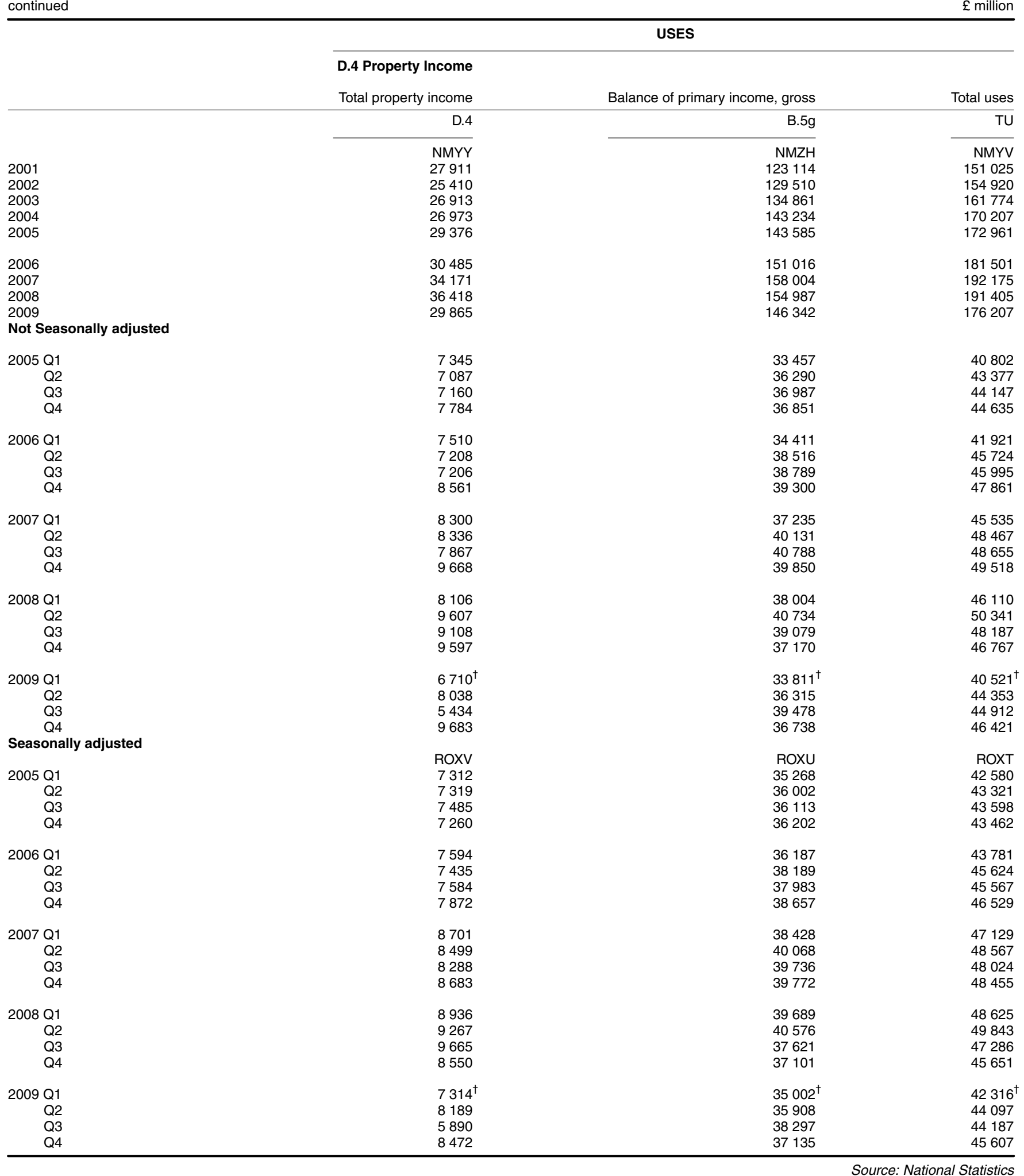




\section{RESOURCES}

D.5 Current taxes on income wealth etc

D.61 Social contributions

D.7 Other current transfers

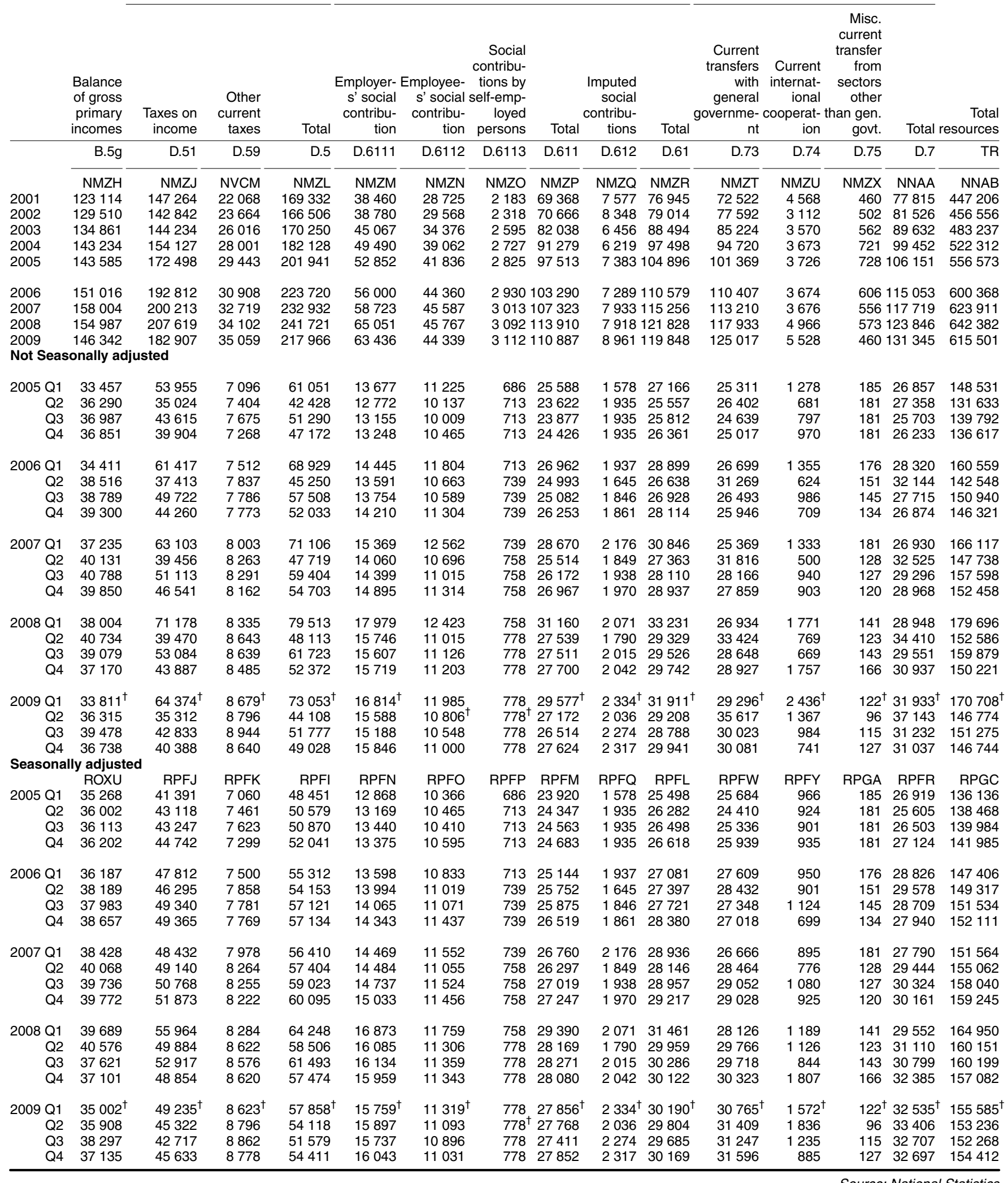


USES

D.7 Other current transfers

\begin{tabular}{|c|c|c|c|c|c|c|c|c|}
\hline & $\begin{array}{r}\text { Current taxes } \\
\text { other than on } \\
\text { income }\end{array}$ & $\begin{array}{r}\text { Social benefits } \\
\text { other than } \\
\text { social transfers } \\
\text { in kind }\end{array}$ & $\begin{array}{r}\text { Current } \\
\text { transfers within } \\
\text { general } \\
\text { government }\end{array}$ & $\begin{array}{r}\text { Current } \\
\text { international } \\
\text { cooperation }\end{array}$ & $\begin{array}{r}\text { Misc. current } \\
\text { transfers to } \\
\text { sectors other } \\
\text { than gen. govt. }\end{array}$ & Total & $\begin{array}{r}\text { Disposable } \\
\text { income, gross }\end{array}$ & Total uses \\
\hline & D59 & D.62 & D.73 & D.74 & D.75 & D.7 & B.6g & $\overline{\mathrm{TU}}$ \\
\hline & EBFQ & NNAD & NNAF & NNAG & NNAI & NNAN & NNAO & NNAB \\
\hline 2001 & 902 & 129591 & 72522 & 2190 & 22131 & 97108 & 219605 & 447206 \\
\hline 2002 & 876 & 136801 & 77592 & 2362 & 27351 & 107625 & 211254 & 456556 \\
\hline 2003 & 842 & 146066 & 85224 & 2433 & 30275 & 118208 & 218121 & 483237 \\
\hline 2004 & 924 & 154314 & 94720 & 3080 & 31178 & 129316 & 237758 & 522312 \\
\hline 2005 & 1022 & 161422 & 101369 & 3255 & 34355 & 139307 & 254822 & 556573 \\
\hline 2006 & 1075 & 167052 & 110407 & 3632 & 34695 & 149100 & 283141 & 600368 \\
\hline 2007 & 1111 & 178379 & 113210 & 3930 & 35878 & 153295 & 291126 & 623911 \\
\hline 2008 & 1147 & 190084 & 117933 & 4292 & 36966 & 159565 & 291586 & 642382 \\
\hline 2009 & 1189 & 210483 & 125017 & 4694 & 40848 & 170899 & 232930 & 615501 \\
\hline \multicolumn{9}{|c|}{ Not Seasonally adjusted } \\
\hline 2005 Q1 & 233 & 37804 & 25311 & 875 & 9138 & 35407 & 75087 & 148531 \\
\hline Q2 & 263 & 39230 & 26402 & 347 & 8159 & 35002 & 57138 & 131633 \\
\hline Q3 & 263 & 40221 & 24639 & 1123 & 8697 & 34545 & 64763 & 139792 \\
\hline Q4 & 263 & 44167 & 25017 & 910 & 8361 & 34353 & 57834 & 136617 \\
\hline 2006 Q1 & 262 & 39822 & 26699 & 1295 & 8862 & 36946 & 83529 & 160559 \\
\hline Q2 & 271 & 40850 & 31269 & 656 & 8163 & 40188 & 61239 & 142548 \\
\hline Q3 & 271 & 42350 & 26493 & 910 & 8891 & 36385 & 71934 & 150940 \\
\hline Q4 & 271 & 44030 & 25946 & 771 & 8779 & 35581 & 66439 & 146321 \\
\hline 2007 Q1 & 271 & 41907 & 25369 & 1355 & 9053 & 35824 & 88115 & 166117 \\
\hline Q2 & 280 & 43789 & 31816 & 814 & 7916 & 40627 & 63042 & 147738 \\
\hline Q3 & 280 & 45224 & 28166 & 859 & 8855 & 37943 & 74151 & 157598 \\
\hline Q4 & 280 & 47459 & 27859 & 902 & 10054 & 38901 & 65818 & 152458 \\
\hline 2008 Q1 & 280 & 43901 & 26934 & 1378 & 10285 & 38699 & 96816 & 179696 \\
\hline Q2 & 289 & 46990 & 33424 & 658 & 8576 & 42752 & 62555 & 152586 \\
\hline Q3 & 289 & 48305 & 28648 & 991 & 8816 & 38546 & 72739 & 159879 \\
\hline Q4 & 289 & 50888 & 28927 & 1265 & 9289 & 39568 & 59476 & 150221 \\
\hline 2009 Q1 & 289 & $49454^{\dagger}$ & $29296^{\dagger}$ & $1366^{\dagger}$ & $10408^{\dagger}$ & $41149^{\dagger}$ & $79816^{\dagger}$ & $170708^{\dagger}$ \\
\hline Q2 & 300 & 51918 & 35617 & 869 & 10815 & 47364 & 47192 & 146774 \\
\hline Q3 & 300 & 53153 & 30023 & 1096 & 9479 & 40708 & 57114 & 151275 \\
\hline Q4 & 300 & 55958 & 30081 & 1363 & 10146 & 41678 & 48808 & 146744 \\
\hline \multicolumn{9}{|c|}{ Seasonally adjusted } \\
\hline 2005 Q1 & 233 & 38957 & 25684 & 691 & 8333 & 34792 & 62154 & 136136 \\
\hline Q2 & 263 & 39919 & 24410 & 401 & 8539 & 33440 & 64846 & 138468 \\
\hline Q3 & 263 & 40424 & 25336 & 1245 & 8774 & 35440 & 63857 & 139984 \\
\hline Q4 & 263 & 42122 & 25939 & 918 & 8709 & 35635 & 63965 & 141985 \\
\hline 2006 Q1 & 262 & 40980 & 27609 & 1125 & 8128 & 36953 & 69211 & 147406 \\
\hline Q2 & 271 & 41442 & 28432 & 782 & 8484 & 37792 & 69812 & 149317 \\
\hline Q3 & 271 & 42400 & 27348 & 969 & 8910 & 37319 & 71544 & 151534 \\
\hline Q4 & 271 & 42230 & 27018 & 756 & 9173 & 37036 & 72574 & 152111 \\
\hline 2007 Q1 & 271 & 43044 & 26666 & 1087 & 8280 & 36081 & 72168 & 151564 \\
\hline Q2 & 280 & 44388 & 28464 & 1020 & 8177 & 37737 & 72657 & 155062 \\
\hline Q3 & 280 & 45242 & 29052 & 914 & 8884 & 38915 & 73603 & 158040 \\
\hline Q4 & 280 & 45705 & 29028 & 909 & 10537 & 40562 & 72698 & 159245 \\
\hline 2008 Q1 & 280 & 45229 & 28126 & 1058 & 9267 & 38547 & 80894 & 164950 \\
\hline Q2 & 289 & 47741 & 29766 & 844 & 8933 & 39638 & 72483 & 160151 \\
\hline Q3 & 289 & 48426 & 29718 & 1076 & 9056 & 39944 & 71540 & 160199 \\
\hline Q4 & 289 & 48689 & 30323 & 1314 & 9710 & 41436 & 66668 & 157082 \\
\hline 2009 Q1 & 289 & $50752^{\dagger}$ & $30765^{\dagger}$ & $1007^{\dagger}$ & $9241^{\dagger}$ & $41089^{\dagger}$ & $63455^{\dagger}$ & $155585^{\dagger}$ \\
\hline Q2 & 300 & 52693 & 31409 & 1032 & 11466 & 43972 & 56271 & 153236 \\
\hline Q3 & 300 & 53311 & 31247 & 1250 & 9714 & 42321 & 56336 & 152268 \\
\hline Q4 & 300 & 53727 & 31596 & 1405 & 10427 & 43517 & 56868 & 154412 \\
\hline
\end{tabular}




\subsection{C}

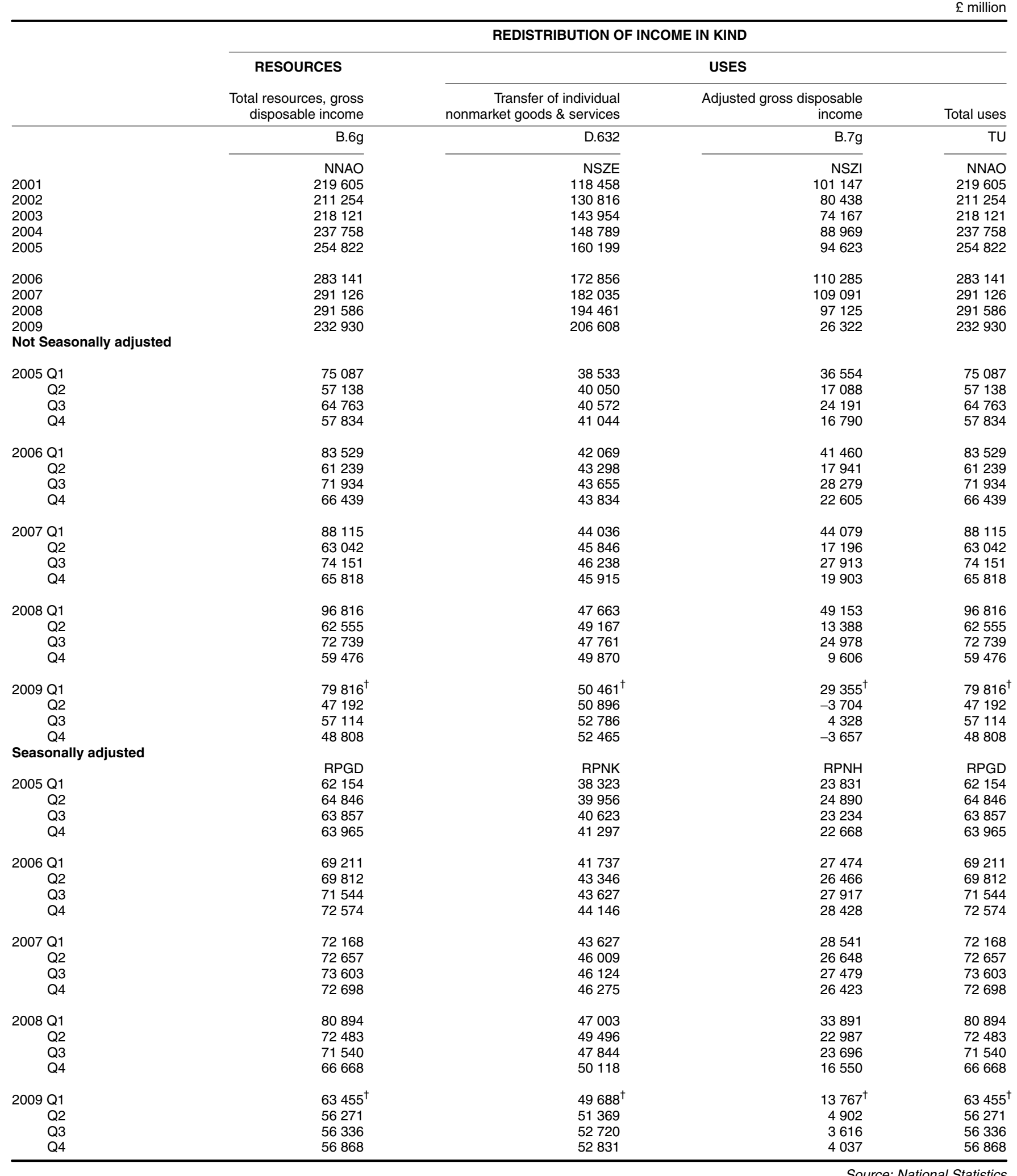


$£$ million

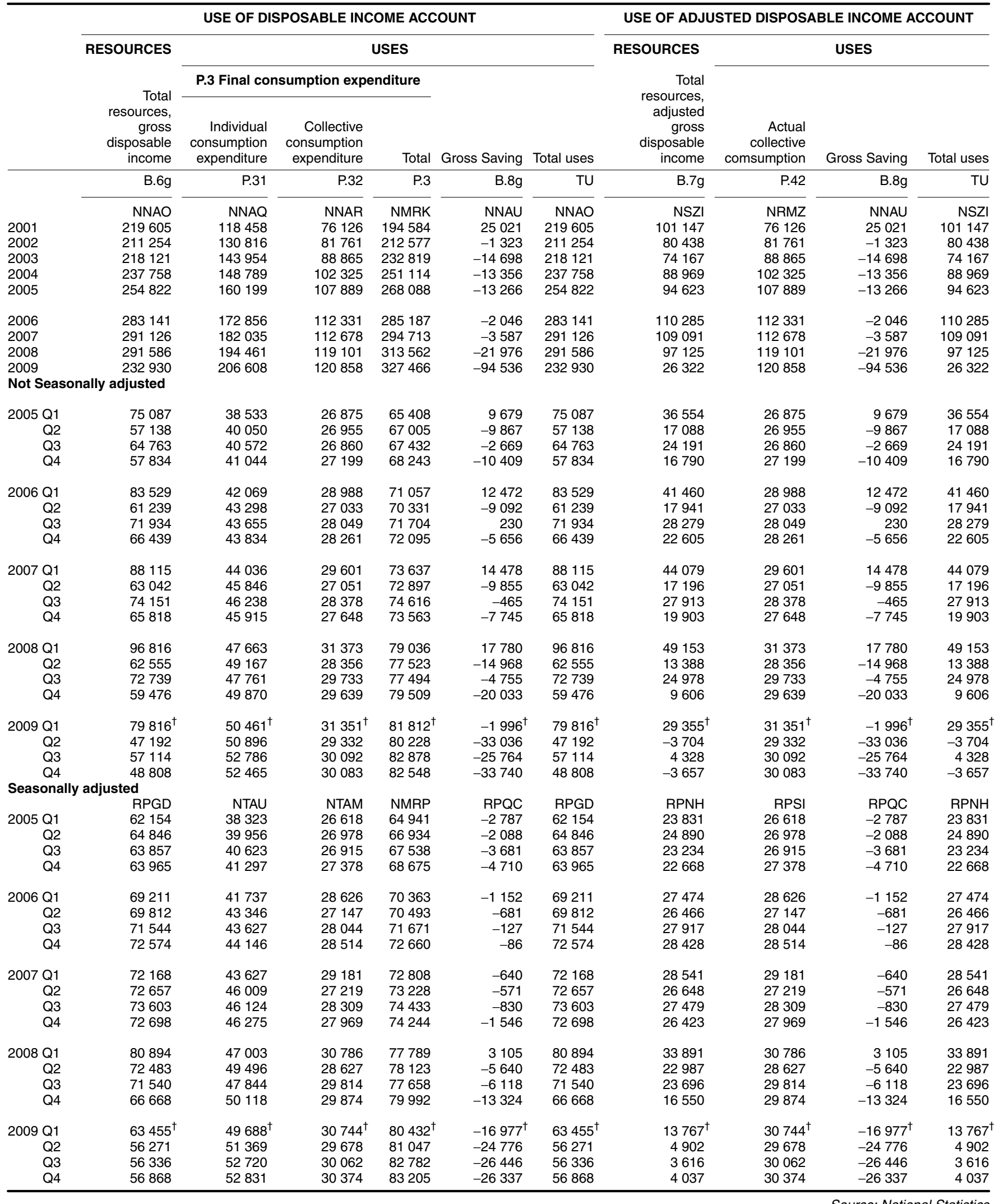




\begin{tabular}{|c|c|c|c|c|c|c|c|c|c|}
\hline & \multicolumn{9}{|c|}{ ACCUMULATION ACCOUNTS } \\
\hline & \multicolumn{9}{|c|}{ CAPITAL ACCOUNT } \\
\hline & \multicolumn{9}{|c|}{ CHANGE IN NET WORTH DUE TO SAVING AND CAPITAL TRANSFERS } \\
\hline & \multicolumn{9}{|c|}{ Changes in liabilities and net worth } \\
\hline & \multicolumn{5}{|c|}{ D.9 Capital transfers receivable } & \multicolumn{3}{|c|}{-D.9 Less capital transfers payable } & $\begin{array}{r}\text { Total change in } \\
\text { liabilities and } \\
\text { net worth }\end{array}$ \\
\hline & B.8g & D.91 & D.92 & D.99 & D.9 & $-\mathrm{D} .92$ & -D.99 & $-\mathrm{D} .9$ & B.10.1g \\
\hline $\begin{array}{l}2001 \\
2002 \\
2003 \\
2004 \\
2005\end{array}$ & $\begin{array}{r}\text { NNAU } \\
25021 \\
-1323 \\
-14698 \\
-13356 \\
-13266\end{array}$ & $\begin{array}{l}\text { NMGI } \\
2396 \\
2381 \\
2416 \\
2881 \\
3150\end{array}$ & $\begin{array}{l}\text { NSZF } \\
4652 \\
6328 \\
7360 \\
6804 \\
7582\end{array}$ & $\begin{array}{r}\text { NNAX } \\
828 \\
1147 \\
5161 \\
3961 \\
4510\end{array}$ & $\begin{array}{r}\text { NNAY } \\
7876 \\
9856 \\
14937 \\
13646 \\
15242\end{array}$ & $\begin{array}{l}-N N A W \\
-11313 \\
-13646 \\
-17335 \\
-16176 \\
-19990\end{array}$ & $\begin{array}{r}- \text { NNBB } \\
-1302 \\
-683 \\
-4494 \\
-3896 \\
-16208\end{array}$ & $\begin{array}{l}-N N B C \\
-12615 \\
-14329 \\
-21829 \\
-20072 \\
-36198\end{array}$ & $\begin{array}{r}\text { NMWG } \\
20282 \\
-5796 \\
-21590 \\
-19782 \\
-34222\end{array}$ \\
\hline $\begin{array}{l}2006 \\
2007 \\
2008 \\
2009 \\
\text { Not Seasc }\end{array}$ & $\begin{array}{r}-2046 \\
-3587 \\
-21976 \\
-94536 \\
\text { adjusted }\end{array}$ & $\begin{array}{r}3575 \\
3867 \\
23864 \\
4415\end{array}$ & $\begin{array}{r}8515 \\
9901 \\
10892 \\
12913\end{array}$ & $\begin{array}{l}2598 \\
2956 \\
4778 \\
1966\end{array}$ & $\begin{array}{l}14688 \\
16724 \\
39534 \\
19294\end{array}$ & $\begin{array}{l}-21163 \\
-24592 \\
-23340 \\
-33171\end{array}$ & $\begin{array}{r}-3561 \\
-2587 \\
-32488 \\
-11715\end{array}$ & $\begin{array}{l}-24724 \\
-27179 \\
-55828 \\
-44886\end{array}$ & $\begin{array}{r}-12082 \\
-14042 \\
-38270 \\
-120128\end{array}$ \\
\hline $\begin{array}{r}2005 \text { Q1 } \\
\text { Q2 } \\
\text { Q3 } \\
\text { Q4 }\end{array}$ & $\begin{array}{r}9679 \\
-9867 \\
-2669 \\
-10409\end{array}$ & $\begin{array}{l}710 \\
804 \\
843 \\
793\end{array}$ & $\begin{array}{l}2326 \\
1437 \\
1636 \\
2183\end{array}$ & $\begin{array}{r}2125 \\
754 \\
885 \\
746\end{array}$ & $\begin{array}{l}5161 \\
2995 \\
3364 \\
3722\end{array}$ & $\begin{array}{l}-6717 \\
-3807 \\
-4247 \\
-5219\end{array}$ & $\begin{array}{r}-1465 \\
-12203 \\
-855 \\
-1685\end{array}$ & $\begin{array}{r}-8182 \\
-16010 \\
-5102 \\
-6904\end{array}$ & $\begin{array}{r}6658 \\
-22882 \\
-4407 \\
-13591\end{array}$ \\
\hline $\begin{array}{r}2006 \text { Q1 } \\
\text { Q2 } \\
\text { Q3 } \\
\text { Q4 }\end{array}$ & $\begin{array}{r}12472 \\
-9092 \\
230 \\
-5656\end{array}$ & $\begin{array}{l}836 \\
904 \\
912 \\
923\end{array}$ & $\begin{array}{l}2633 \\
1951 \\
2022 \\
1909\end{array}$ & $\begin{array}{l}648 \\
497 \\
592 \\
861\end{array}$ & $\begin{array}{l}4117 \\
3352 \\
3526 \\
3693\end{array}$ & $\begin{array}{l}-6928 \\
-4389 \\
-4807 \\
-5039\end{array}$ & $\begin{array}{r}-629 \\
-1607 \\
-636 \\
-689\end{array}$ & $\begin{array}{l}-7557 \\
-5996 \\
-5443 \\
-5728\end{array}$ & $\begin{array}{r}9032 \\
-11736 \\
-1687 \\
-7691\end{array}$ \\
\hline $\begin{array}{r}2007 \text { Q1 } \\
\text { Q2 } \\
\text { Q3 } \\
\text { Q4 }\end{array}$ & $\begin{array}{r}14478 \\
-9855 \\
-465 \\
-7745\end{array}$ & $\begin{array}{r}879 \\
966 \\
1063 \\
959\end{array}$ & $\begin{array}{l}2545 \\
2370 \\
2421 \\
2565\end{array}$ & $\begin{array}{r}900 \\
367 \\
515 \\
1174\end{array}$ & $\begin{array}{l}4324 \\
3703 \\
3999 \\
4698\end{array}$ & $\begin{array}{l}-8554 \\
-4758 \\
-5426 \\
-5854\end{array}$ & $\begin{array}{r}-1051 \\
-269 \\
-240 \\
-1027\end{array}$ & $\begin{array}{l}-9605 \\
-5027 \\
-5666 \\
-6881\end{array}$ & $\begin{array}{r}9197 \\
-11179 \\
-2132 \\
-9928\end{array}$ \\
\hline $\begin{array}{r}2009 \text { Q1 } \\
\text { Q2 } \\
\text { Q3 } \\
\text { Q4 }\end{array}$ & $\begin{array}{r}-1996^{\dagger} \\
-33036 \\
-25764 \\
-33740\end{array}$ & $\begin{array}{r}2164^{\dagger} \\
573 \\
1062 \\
616\end{array}$ & $\begin{array}{l}3422^{\dagger} \\
3117 \\
3200 \\
3174\end{array}$ & $\begin{array}{c}1013^{\dagger} \\
265 \\
248 \\
440\end{array}$ & $\begin{array}{l}6599^{\dagger} \\
3955 \\
4510 \\
4230\end{array}$ & $\begin{array}{l}-11811^{\dagger} \\
-6649 \\
-7062 \\
-7649\end{array}$ & $\begin{array}{c}-6623^{\dagger} \\
-203 \\
-462 \\
-4427\end{array}$ & $\begin{array}{r}-18434^{\dagger} \\
-6852 \\
-7524 \\
-12076\end{array}$ & $\begin{array}{l}-13831^{\dagger} \\
-35933 \\
-28778 \\
-41586\end{array}$ \\
\hline $\begin{array}{r}\text { Seasonal } \\
2005 \text { Q1 } \\
\text { Q2 } \\
\text { Q3 } \\
\text { Q4 }\end{array}$ & $\begin{array}{l}\text { RPQC } \\
-2787 \\
-2088 \\
-3681 \\
-4710\end{array}$ & $\begin{array}{r}\text { RNGQ } \\
710 \\
804 \\
843 \\
793\end{array}$ & $\begin{array}{r}\text { RPUO } \\
1890 \\
1656 \\
1776 \\
2260\end{array}$ & $\begin{array}{r}\text { RPUR } \\
2196 \\
678 \\
967 \\
669\end{array}$ & $\begin{array}{r}\text { RPUL } \\
4796 \\
3138 \\
3586 \\
3722\end{array}$ & $\begin{array}{l}\text {-RPUY } \\
-6162 \\
-4207 \\
-4411 \\
-5210\end{array}$ & $\begin{array}{r}\text {-RPVB } \\
-1465 \\
-12203 \\
-855 \\
-1685\end{array}$ & $\begin{array}{r}\text {-RPUV } \\
-7627 \\
-16410 \\
-5266 \\
-6895\end{array}$ & $\begin{array}{r}\text { RPUJ } \\
-5618 \\
-15360 \\
-5361 \\
-7883\end{array}$ \\
\hline $\begin{array}{r}2006 \text { Q1 } \\
\text { Q2 } \\
\text { Q3 } \\
\text { Q4 }\end{array}$ & $\begin{array}{r}-1152 \\
-681 \\
-127 \\
-86\end{array}$ & $\begin{array}{l}836 \\
904 \\
912 \\
923\end{array}$ & $\begin{array}{l}2182 \\
2190 \\
2205 \\
1938\end{array}$ & $\begin{array}{l}636 \\
612 \\
708 \\
642\end{array}$ & $\begin{array}{l}3654 \\
3706 \\
3825 \\
3503\end{array}$ & $\begin{array}{l}-6347 \\
-4795 \\
-4993 \\
-5028\end{array}$ & $\begin{array}{r}-629 \\
-1607 \\
-636 \\
-689\end{array}$ & $\begin{array}{l}-6976 \\
-6402 \\
-5629 \\
-5717\end{array}$ & $\begin{array}{l}-4474 \\
-3377 \\
-1931 \\
-2300\end{array}$ \\
\hline $\begin{array}{r}2007 \text { Q1 } \\
\text { Q2 } \\
\text { Q3 } \\
\text { Q4 }\end{array}$ & $\begin{array}{r}-640 \\
-571 \\
-830 \\
-1546\end{array}$ & $\begin{array}{r}879 \\
966 \\
1063 \\
959\end{array}$ & $\begin{array}{l}2097 \\
2570 \\
2640 \\
2594\end{array}$ & $\begin{array}{r}751 \\
443 \\
573 \\
1189\end{array}$ & $\begin{array}{l}3727 \\
3979 \\
4276 \\
4742\end{array}$ & $\begin{array}{l}-8044 \\
-5010 \\
-5658 \\
-5880\end{array}$ & $\begin{array}{r}-1051 \\
-269 \\
-240 \\
-1027\end{array}$ & $\begin{array}{l}-9095 \\
-5279 \\
-5898 \\
-6907\end{array}$ & $\begin{array}{l}-6008 \\
-1871 \\
-2452 \\
-3711\end{array}$ \\
\hline $\begin{array}{r}2008 \text { Q1 } \\
\text { Q2 } \\
\text { Q3 } \\
\text { Q4 }\end{array}$ & $\begin{array}{r}3105 \\
-5640 \\
-6118 \\
-13324\end{array}$ & $\begin{array}{r}902 \\
837 \\
16569 \\
5556\end{array}$ & $\begin{array}{l}2887 \\
2705 \\
2552 \\
2748\end{array}$ & $\begin{array}{r}3264 \\
517 \\
230 \\
767\end{array}$ & $\begin{array}{r}7053 \\
4059 \\
19351 \\
9071\end{array}$ & $\begin{array}{l}-8775 \\
-4846 \\
-4557 \\
-5162\end{array}$ & $\begin{array}{r}-5044 \\
-323 \\
-19029 \\
-8092\end{array}$ & $\begin{array}{r}-13819 \\
-5169 \\
-23586 \\
-13254\end{array}$ & $\begin{array}{r}-3661 \\
-6750 \\
-10353 \\
-17507\end{array}$ \\
\hline $\begin{array}{r}2009 \text { Q1 } \\
\text { Q2 } \\
\text { Q3 } \\
\text { Q4 }\end{array}$ & $\begin{array}{l}-16977^{\dagger} \\
-24776 \\
-26446 \\
-26337\end{array}$ & $\begin{array}{r}2164^{\dagger} \\
573 \\
1062 \\
616\end{array}$ & $\begin{array}{l}2835^{\dagger} \\
3264 \\
3463 \\
3351\end{array}$ & $\begin{array}{l}995^{\dagger} \\
318 \\
303 \\
350\end{array}$ & $\begin{array}{l}5994^{\dagger} \\
4155 \\
4828 \\
4317\end{array}$ & $\begin{array}{r}-11194^{\dagger} \\
-6846 \\
-7332 \\
-7799\end{array}$ & $\begin{array}{c}-6623^{\dagger} \\
-203 \\
-462 \\
-4427\end{array}$ & $\begin{array}{r}-17817^{\dagger} \\
-7049 \\
-7794 \\
-12226\end{array}$ & $\begin{array}{l}-28800^{\dagger} \\
-27670 \\
-29412 \\
-34246\end{array}$ \\
\hline
\end{tabular}




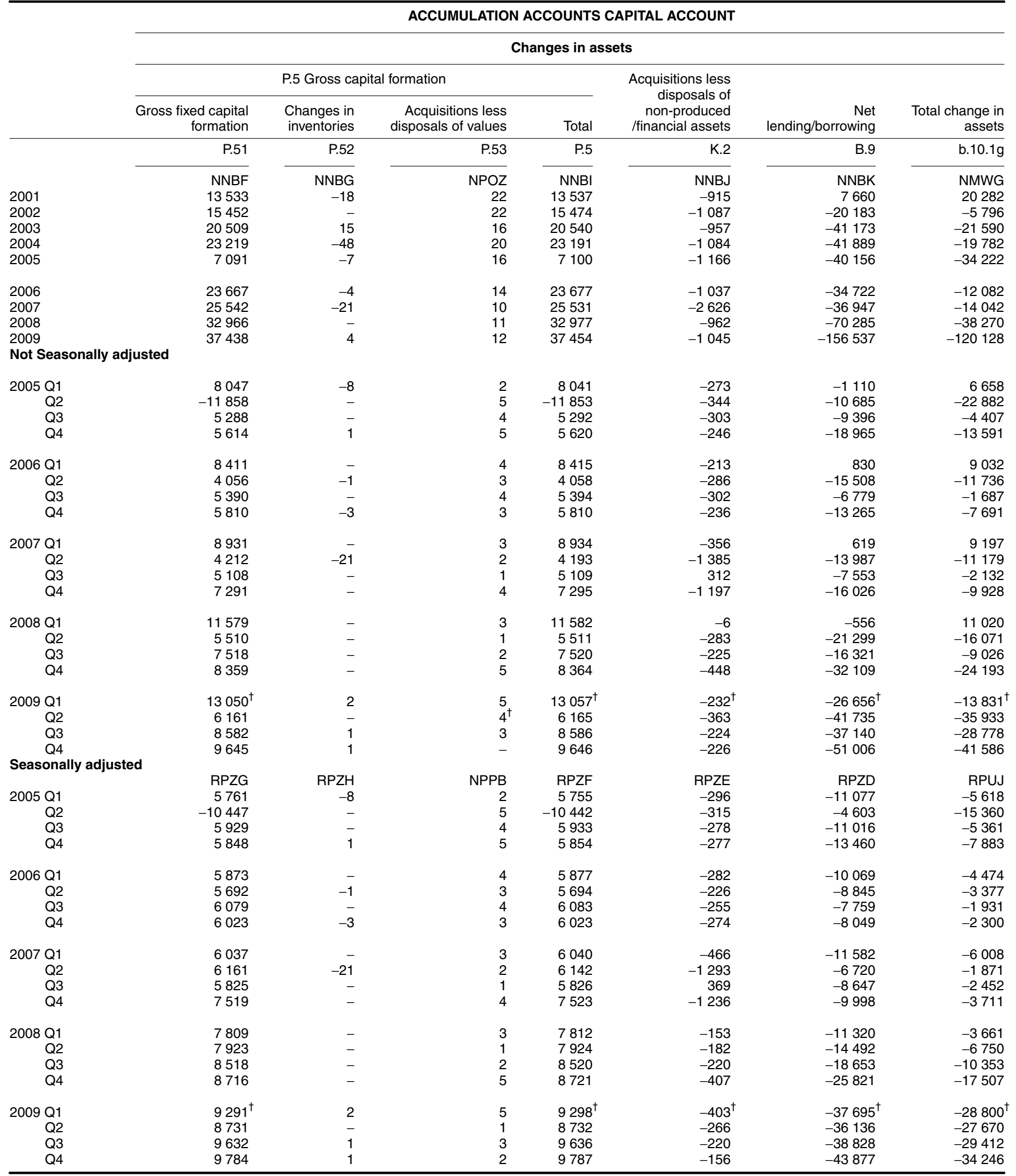




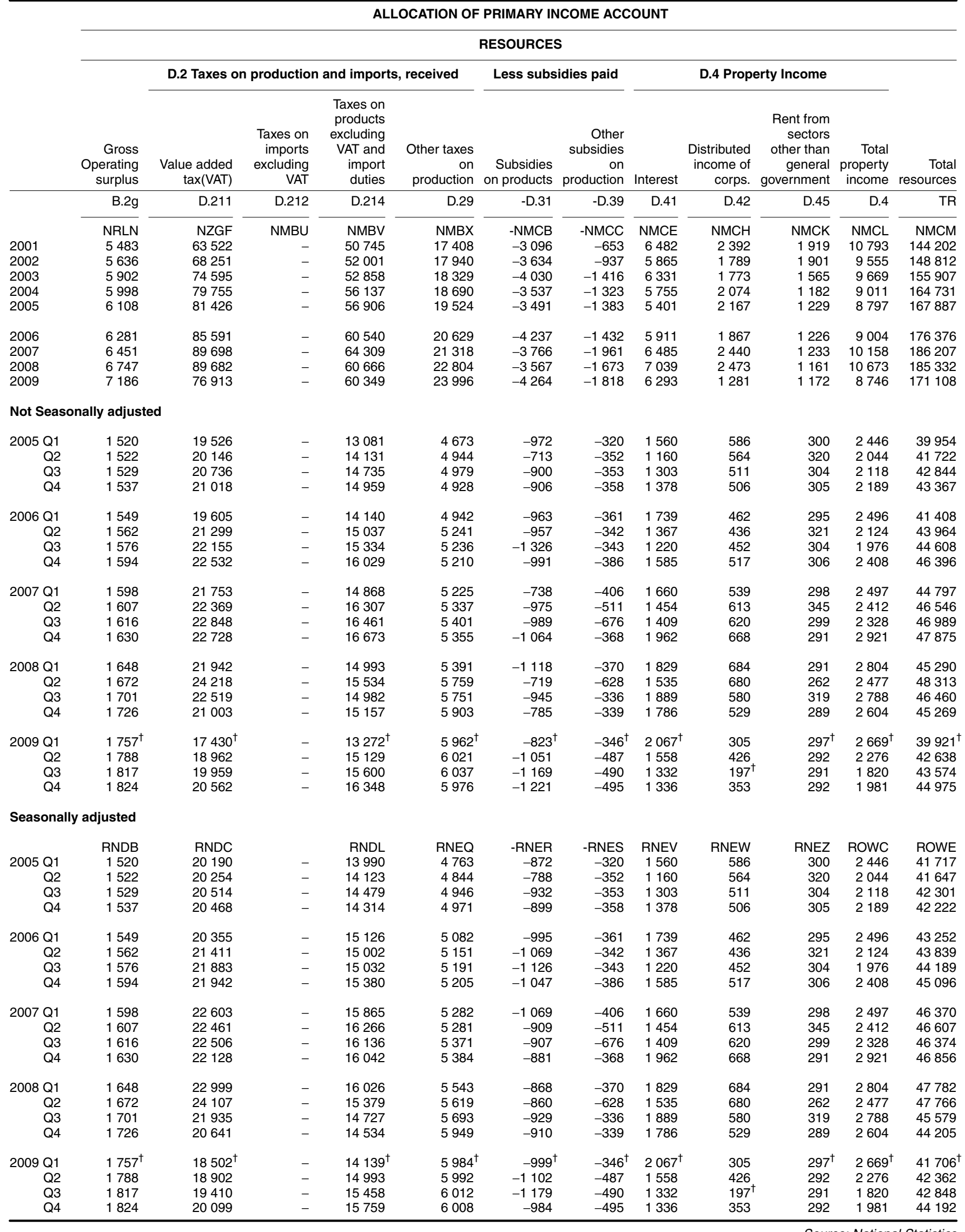


USES

D.4 Property Income

Total property income Balance of primary income, gross Total uses

\begin{tabular}{lr} 
& Total property incom \\
\hline & D. \\
\cline { 2 - 3 } 2001 & NUHA \\
2002 & 2366 \\
2003 & 2142 \\
2004 & 2242 \\
2005 & 2321 \\
2006 & 25907 \\
2007 & \\
2008 & 2683 \\
2009 & 3045 \\
& 3255 \\
& 2683
\end{tabular}

\begin{tabular}{rr} 
B.5g & TU \\
\cline { 2 - 2 } NRLP & NMCM \\
120541 & 144202 \\
127383 & 148812 \\
133486 & 155907 \\
141519 & 164731 \\
141980 & 167887 \\
149545 & \\
155756 & 176376 \\
152777 & 186207 \\
144275 & 185332 \\
& 171108
\end{tabular}

Not Seasonally adjusted

2005 Q1

Q2

6369

6324

6262

6952

33585

35398

36582

36415

39954

41722

42844

2006 Q1

Q2

6498

6374

34910

37590

43367

Q4

6469

38139

41408

38906

43964

44608

46396

2007 Q1

7419

37378

44797

7440

39106

46546

7047

39942

46989

Q3

8545

47875

2008 Q1

7093

39330

45290

8624

38197

39689

38383

48313

8077

36508

46460

45269

2009 Q1

$5783^{\dagger}$

$34138^{\dagger}$

35249

38950

$39921^{\dagger}$

7389

4624
9037

42638

43574

35938

44975

Seasonally adjusted

2005 Q1

Q2

RNHE

6456
6456

6456

6446

ROWF

35261

35191

35752

35776

ROWE

41717

41647

42301

42222

2006 Q1
Q2
Q3

6663

6527

36589

37312

43252

6781

37408

43839

6860

44189

38236

45096

2007 Q1
Q2
Q3

7857

38513

39052

38974

46370

7555

38974
39217

46607

46374

7639

46856

2008 Q1

7984

39798

39536

47782

8230

36964

47766

8615

45579

36479

44205

2009 Q1

$6487^{\dagger}$

$35219^{\dagger}$

34896

37747

$41706^{\dagger}$

42362

7466

5101
7779

36413

42848

44192

Source: National Statistics 
RESOURCES

\begin{tabular}{|c|c|c|c|c|c|c|c|c|c|c|c|c|c|c|c|}
\hline & \multirow[b]{2}{*}{$\begin{array}{l}\text { Balance } \\
\text { of gross } \\
\text { primary } \\
\text { incomes }\end{array}$} & \multicolumn{3}{|c|}{$\begin{array}{l}\text { D.5 Current taxes on income, } \\
\text { wealth etc }\end{array}$} & \multicolumn{6}{|c|}{ D.61 Social contributions } & \multicolumn{4}{|c|}{ D.7 Other current transfers } & \\
\hline & & $\begin{array}{l}\text { Taxes on } \\
\text { income }\end{array}$ & $\begin{array}{r}\text { Other } \\
\text { current } \\
\text { taxes }\end{array}$ & Total & $\begin{array}{r}\text { Employer- } \\
\text { s' social } \\
\text { contribu- } \\
\text { tion }\end{array}$ & $\begin{array}{c}\text { Employee- } \\
\text { s'social } \\
\text { contribu- } \\
\text { tion }\end{array}$ & $\begin{array}{r}\text { Social } \\
\text { contribu- } \\
\text { tions by } \\
\text { self and } \\
\text { non } \\
\text { employed } \\
\text { persons }\end{array}$ & Total & $\begin{array}{r}\text { Imputed } \\
\text { social } \\
\text { sontribu- } \\
\text { tions }\end{array}$ & Total & $\begin{array}{r}\text { Current } \\
\text { transfers } \\
\text { with } \\
\text { general } \\
\text { governme- } \\
\text { nt }\end{array}$ & $\begin{array}{c}\text { Current } \\
\text { internat- } \\
\text { ional } \\
\text { cooperat- } t \\
\text { ion }\end{array}$ & $\begin{array}{r}\text { Misc. } \\
\text { current } \\
\text { transfer } \\
\text { from } \\
\text { sectors } \\
\text { other } \\
\text { han gen. } \\
\text { govt. }\end{array}$ & & $\begin{array}{r}\text { Total } \\
\text { resources }\end{array}$ \\
\hline & B.5g & D.51 & D.59 & D.5 & D.6111 & D.6112 & D.6113 & D.611 & D.612 & D.61 & D.73 & D.74 & D.75 & D.7 & $\overline{T R}$ \\
\hline & NRLP & NMCU & NMCV & NMCP & NMCY & NMDB & NMDE & NMCX & QYJS & NMCW & NMDK & NMDL & NMEZ I & NMDI & NMDN \\
\hline 2001 & 120541 & 147264 & 6920 & 154184 & 38460 & 28116 & 2183 & 68759 & 5620 & 74379 & - & 4568 & 460 & 5028 & 354132 \\
\hline 2002 & 127383 & 142842 & 7133 & 149975 & 38780 & 28931 & 2318 & 70029 & 6282 & 76311 & - & 3112 & 502 & 3614 & 357283 \\
\hline 2003 & 133486 & 144234 & 7534 & 151768 & 45067 & 33717 & 2595 & 81379 & 4311 & 85690 & - & 3570 & 562 & 4132 & 375076 \\
\hline 2004 & 141519 & 154127 & 7991 & 162118 & 49490 & 38359 & 2727 & 90576 & 3997 & 94573 & - & 3604 & 721 & 4325 & 402535 \\
\hline 2005 & 141980 & 172498 & 8330 & 180828 & 52852 & 41078 & 2825 & 96755 & 5073 & 101828 & - & 3668 & 728 & 4396 & 429032 \\
\hline 2007 & 155756 & 200213 & 9344 & 209557 & 58723 & 44746 & 3013 & 106482 & 5369 & 111851 & - & 3592 & 556 & 4148 & 481312 \\
\hline 2008 & 152777 & 207619 & 9629 & 217248 & 65051 & 44889 & 3092 & 113032 & 5193 & 118225 & - & 4862 & 573 & 5435 & 493685 \\
\hline 2009 & 144275 & 182907 & 9810 & 192717 & 63436 & 43417 & 3112 & 109965 & 6056 & 116021 & - & 5418 & 460 & 5878 & 458891 \\
\hline
\end{tabular}

Not Seasonally adjusted

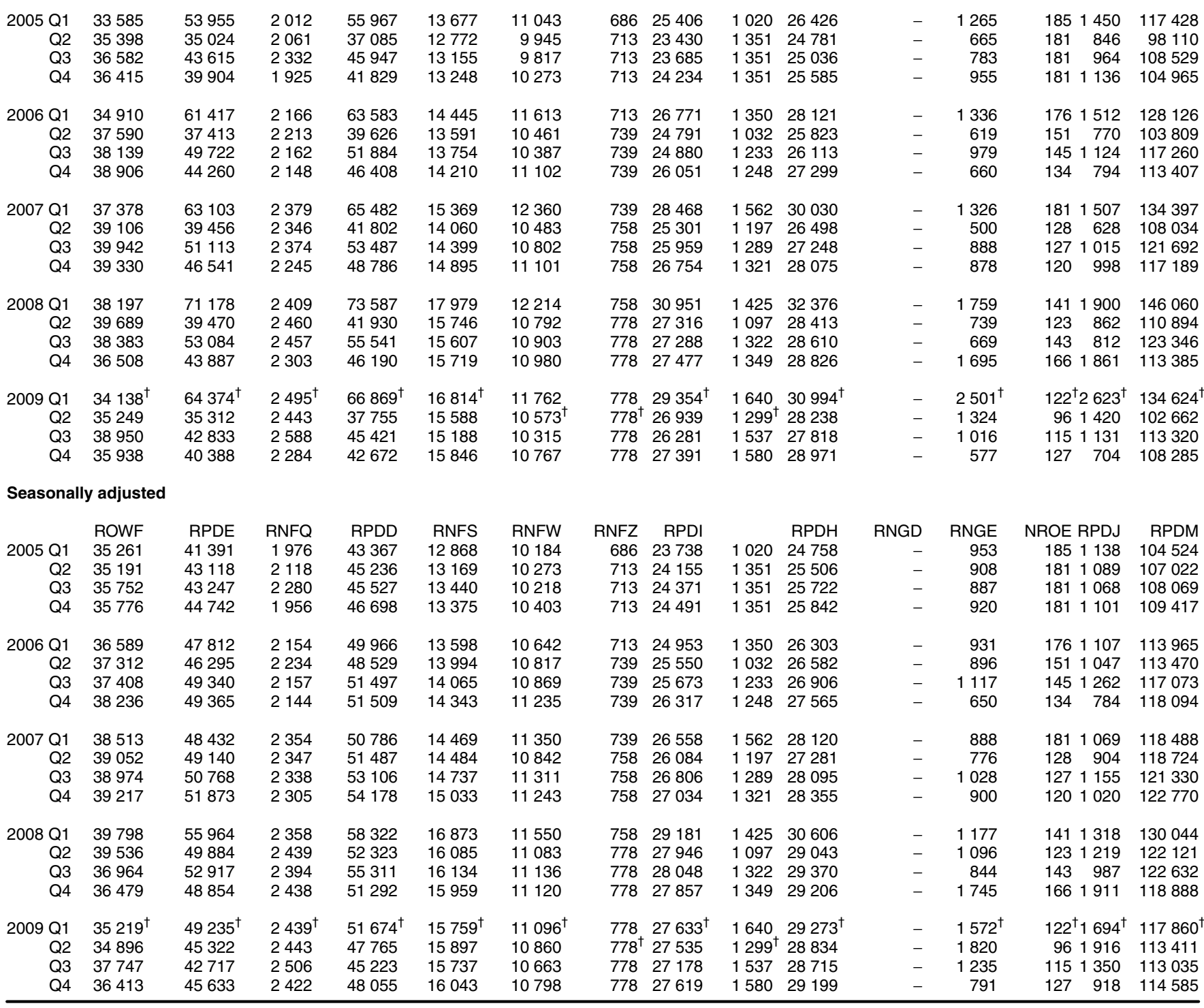




\section{USES}

D.7 Other current transfers

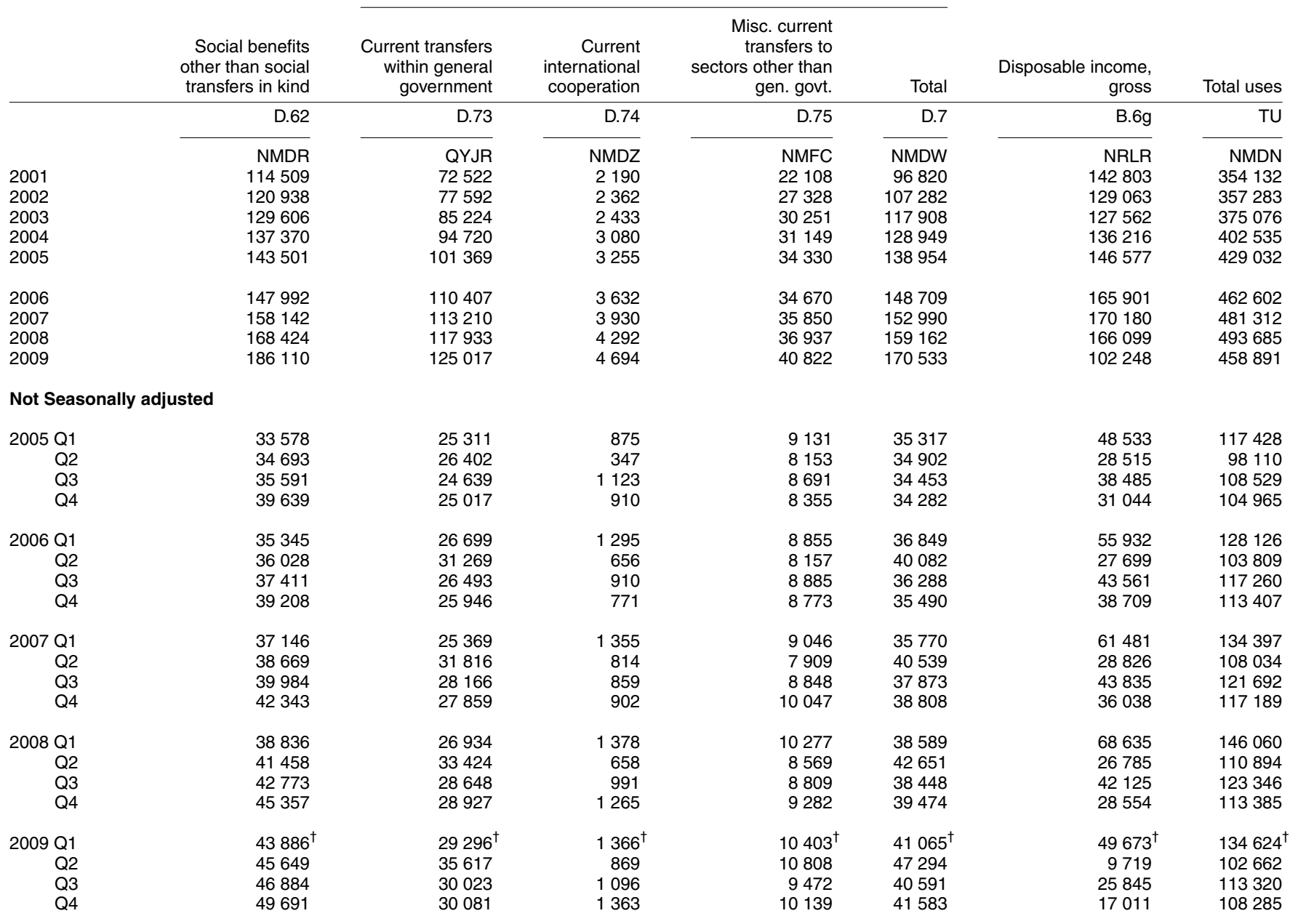

Seasonally adjusted

$\begin{array}{rr}2005 \text { Q1 } & \text { RPDO } \\ \text { Q2 } & 3473 \\ \text { Q3 } & 3538 \\ \text { Q4 } & 35794 \\ & 37594\end{array}$

\begin{tabular}{|c|c|c|c|c|c|}
\hline RNHL & RPDV & RNHS & RPDT & RPDN & RPDM \\
\hline 25684 & 691 & 8326 & 34701 & 35092 & 104524 \\
\hline 24410 & 401 & 8533 & 33344 & 38296 & 107022 \\
\hline 25336 & 1245 & 8768 & 35349 & 36926 & 108069 \\
\hline 25939 & 918 & 8703 & 35560 & 36263 & 109417 \\
\hline 27609 & 1125 & 8121 & 36855 & 40607 & 113965 \\
\hline 28432 & 782 & 8478 & 37692 & 39158 & 113470 \\
\hline 27348 & 969 & 8904 & 37221 & 42391 & 117073 \\
\hline 27018 & 756 & 9167 & 36941 & 43745 & 118094 \\
\hline 26666 & 1087 & 8273 & 36026 & 44179 & 118488 \\
\hline 28464 & 1020 & 8170 & 37654 & 41802 & 118724 \\
\hline 29052 & 914 & 8877 & 38843 & 42485 & 121330 \\
\hline 29028 & 909 & 10530 & 40467 & 41714 & 122770 \\
\hline 28126 & 1058 & 9259 & 38443 & 51437 & 130044 \\
\hline 29766 & 844 & 8926 & 39536 & 40376 & 122121 \\
\hline 29718 & 1076 & 9049 & 39843 & 39895 & 122632 \\
\hline 30323 & 1314 & 9703 & 41340 & 34390 & 118888 \\
\hline $30765^{\dagger}$ & $1007^{\dagger}$ & $9236^{\dagger}$ & $41008^{\dagger}$ & $31668^{\dagger}$ & 11786 \\
\hline 31409 & 1032 & 11459 & 43900 & 23087 & 11341 \\
\hline 31247 & 1250 & 9707 & 42204 & 23789 & 11303 \\
\hline 31596 & 1405 & 10420 & 43421 & 23704 & 11458 \\
\hline
\end{tabular}




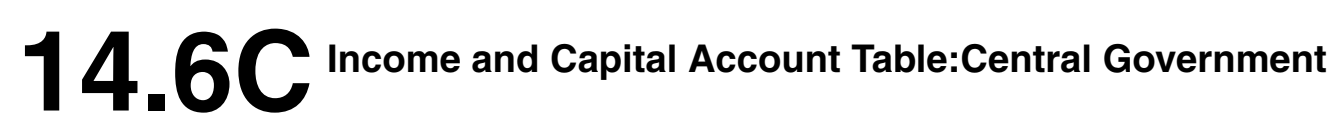

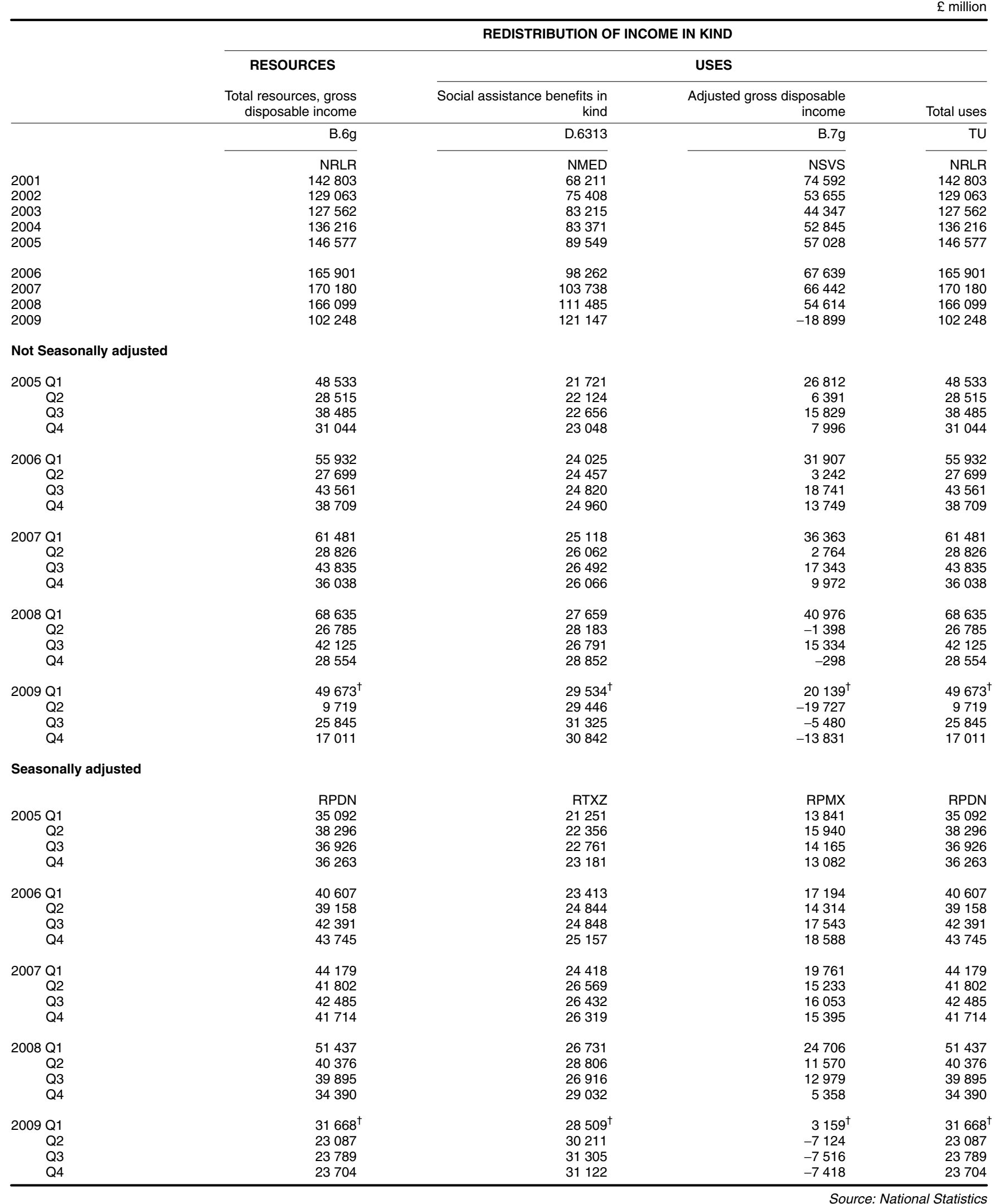


$£$ million

\begin{tabular}{|c|c|c|c|c|c|c|c|c|c|c|}
\hline & \multicolumn{6}{|c|}{ USE OF DISPOSABLE INCOME ACCOUNT } & \multicolumn{4}{|c|}{ USE OF ADJUSTED DISPOSABLE INCOME ACCOUNT } \\
\hline & \multirow{3}{*}{$\begin{array}{r}\text { RESOURCES } \\
\text { Total } \\
\text { resources, } \\
\text { gross } \\
\text { disposable } \\
\text { income }\end{array}$} & \multicolumn{5}{|c|}{ USES } & \multirow{3}{*}{$\begin{array}{r}\text { RESOURCES } \\
\text { Total } \\
\text { resources, } \\
\text { adjusted } \\
\text { gross } \\
\text { disposable } \\
\text { income }\end{array}$} & \multicolumn{3}{|c|}{ USES } \\
\hline & & \multicolumn{3}{|c|}{ P.3 Final consumption expenditure } & & & & \multirow[b]{2}{*}{$\begin{array}{r}\text { Actual } \\
\text { collective } \\
\text { comsumption }\end{array}$} & \multirow[b]{2}{*}{ Gross Saving } & \multirow[b]{2}{*}{ Total uses } \\
\hline & & $\begin{array}{r}\text { Individual } \\
\text { consumption } \\
\text { expenditure }\end{array}$ & $\begin{array}{r}\text { Collective } \\
\text { consumption } \\
\text { expenditure }\end{array}$ & Total & Gross Saving & Total uses & & & & \\
\hline $\begin{array}{l}2001 \\
2002 \\
2003 \\
2004 \\
2005\end{array}$ & $\begin{array}{r}\text { NRLR } \\
142803 \\
129063 \\
127562 \\
136216 \\
146577\end{array}$ & $\begin{array}{l}\text { NMED } \\
68211 \\
75408 \\
83215 \\
83371 \\
89549\end{array}$ & $\begin{array}{c}\text { NMEE } \\
50567 \\
54940 \\
59443 \\
68903 \\
71780\end{array}$ & $\begin{array}{r}\text { NMBJ } \\
118778 \\
130348 \\
142658 \\
152274 \\
161329\end{array}$ & $\begin{array}{r}\text { NRLS } \\
24025 \\
-1285 \\
-15096 \\
-16058 \\
-14752\end{array}$ & $\begin{array}{r}\text { NRLR } \\
142803 \\
129063 \\
127562 \\
136216 \\
146577\end{array}$ & $\begin{array}{r}\text { NSVS } \\
74592 \\
53655 \\
44347 \\
52845 \\
57028\end{array}$ & $\begin{array}{c}\text { NMEE } \\
50567 \\
54940 \\
59443 \\
68903 \\
71780\end{array}$ & $\begin{array}{r}\text { NRLS } \\
24025 \\
-1285 \\
-15096 \\
-16058 \\
-14752\end{array}$ & $\begin{array}{r}\text { NSVS } \\
74592 \\
53655 \\
44347 \\
52845 \\
57028\end{array}$ \\
\hline $\begin{array}{l}2006 \\
2007 \\
2008 \\
2009\end{array}$ & $\begin{array}{l}165901 \\
170180 \\
166099 \\
102248\end{array}$ & $\begin{array}{r}98262 \\
103738 \\
111485 \\
121147\end{array}$ & $\begin{array}{l}75166 \\
74041 \\
78661 \\
78773\end{array}$ & $\begin{array}{l}173428 \\
177779 \\
190146 \\
199920\end{array}$ & $\begin{array}{r}-7527 \\
-7599 \\
-24047 \\
-97672\end{array}$ & $\begin{array}{l}165901 \\
170180 \\
166099 \\
102248\end{array}$ & $\begin{array}{r}67639 \\
66442 \\
54614 \\
-18899\end{array}$ & $\begin{array}{l}75166 \\
74041 \\
78661 \\
78773\end{array}$ & $\begin{array}{r}-7527 \\
-7599 \\
-24047 \\
-97672\end{array}$ & $\begin{array}{r}67639 \\
66442 \\
54614 \\
-18899\end{array}$ \\
\hline \multicolumn{11}{|c|}{ Not Seasonally adjusted } \\
\hline $\begin{array}{r}2005 \text { Q1 } \\
\text { Q2 } \\
\text { Q3 } \\
\text { Q4 }\end{array}$ & $\begin{array}{l}48533 \\
28515 \\
38485 \\
31044\end{array}$ & $\begin{array}{l}21721 \\
22124 \\
22656 \\
23048\end{array}$ & $\begin{array}{l}18121 \\
17853 \\
17761 \\
18045\end{array}$ & $\begin{array}{l}39842 \\
39977 \\
40417 \\
41093\end{array}$ & $\begin{array}{r}8691 \\
-11462 \\
-1932 \\
-10049\end{array}$ & $\begin{array}{l}48533 \\
28515 \\
38485 \\
31044\end{array}$ & $\begin{array}{r}26812 \\
6391 \\
15829 \\
7996\end{array}$ & $\begin{array}{l}18121 \\
17853 \\
17761 \\
18045\end{array}$ & $\begin{array}{r}8691 \\
-11462 \\
-1932 \\
-10049\end{array}$ & $\begin{array}{r}26812 \\
6391 \\
15829 \\
7996\end{array}$ \\
\hline $\begin{array}{r}2006 \text { Q1 } \\
\text { Q2 } \\
\text { Q3 } \\
\text { Q4 }\end{array}$ & $\begin{array}{l}55932 \\
27699 \\
43561 \\
38709\end{array}$ & $\begin{array}{l}24025 \\
24457 \\
24820 \\
24960\end{array}$ & $\begin{array}{l}19802 \\
17733 \\
18730 \\
18901\end{array}$ & $\begin{array}{l}43827 \\
42190 \\
43550 \\
43861\end{array}$ & $\begin{array}{r}12105 \\
-14491 \\
11 \\
-5152\end{array}$ & $\begin{array}{l}55932 \\
27699 \\
43561 \\
38709\end{array}$ & $\begin{array}{r}31907 \\
3242 \\
18741 \\
13749\end{array}$ & $\begin{array}{l}19802 \\
17733 \\
18730 \\
18901\end{array}$ & $\begin{array}{r}12105 \\
-14491 \\
11 \\
-5152\end{array}$ & $\begin{array}{r}31907 \\
3242 \\
18741 \\
13749\end{array}$ \\
\hline $\begin{array}{r}2007 \text { Q1 } \\
\text { Q2 } \\
\text { Q3 } \\
\text { Q4 }\end{array}$ & $\begin{array}{l}61481 \\
28826 \\
43835 \\
36038\end{array}$ & $\begin{array}{l}25118 \\
26062 \\
26492 \\
26066\end{array}$ & $\begin{array}{l}20229 \\
17316 \\
18645 \\
17851\end{array}$ & $\begin{array}{l}45347 \\
43378 \\
45137 \\
43917\end{array}$ & $\begin{array}{r}16134 \\
-14552 \\
-1302 \\
-7879\end{array}$ & $\begin{array}{l}61481 \\
28826 \\
43835 \\
36038\end{array}$ & $\begin{array}{r}36363 \\
2764 \\
17343 \\
9972\end{array}$ & $\begin{array}{l}20229 \\
17316 \\
18645 \\
17851\end{array}$ & $\begin{array}{r}16134 \\
-14552 \\
-1302 \\
-7879\end{array}$ & $\begin{array}{r}36363 \\
2764 \\
17343 \\
9972\end{array}$ \\
\hline $\begin{array}{r}2009 \text { Q1 } \\
\text { Q2 } \\
\text { Q3 } \\
\text { Q4 }\end{array}$ & $\begin{array}{r}49673^{\dagger} \\
9719 \\
25845 \\
17011\end{array}$ & $\begin{array}{l}29534^{\dagger} \\
29446 \\
31325 \\
30842\end{array}$ & $\begin{array}{l}20995^{\dagger} \\
18826 \\
19542 \\
19410\end{array}$ & $\begin{array}{l}50529^{\dagger} \\
48272 \\
50867 \\
50252\end{array}$ & $\begin{array}{r}-856^{\dagger} \\
-38553 \\
-25022 \\
-33241\end{array}$ & $\begin{array}{r}49673^{\dagger} \\
9719 \\
25845 \\
17011\end{array}$ & $\begin{array}{r}20139^{\dagger} \\
-19727 \\
-5480 \\
-13831\end{array}$ & $\begin{array}{l}20995^{\dagger} \\
18826 \\
19542 \\
19410\end{array}$ & $\begin{array}{r}-856^{\dagger} \\
-38553 \\
-25022 \\
-33241\end{array}$ & $\begin{array}{r}20139 \dagger \\
-19727 \\
-5480 \\
-13831\end{array}$ \\
\hline \multicolumn{11}{|c|}{ Seasonally adjusted } \\
\hline $\begin{array}{r}2005 \text { Q1 } \\
\text { Q2 } \\
\text { Q3 } \\
\text { Q4 }\end{array}$ & $\begin{array}{l}\text { RPDN } \\
35092 \\
38296 \\
36926 \\
36263\end{array}$ & $\begin{array}{r}\text { RTXZ } \\
21251 \\
22356 \\
22761 \\
23181\end{array}$ & $\begin{array}{r}\text { RTYC } \\
17728 \\
18041 \\
17844 \\
18167\end{array}$ & $\begin{array}{l}\text { NMRE } \\
38979 \\
40397 \\
40605 \\
41348\end{array}$ & $\begin{array}{l}\text { RPPN } \\
-3887 \\
-2101 \\
-3679 \\
-5085\end{array}$ & $\begin{array}{r}\text { RPDN } \\
35092 \\
38296 \\
36926 \\
36263\end{array}$ & $\begin{array}{c}\text { RPMX } \\
13841 \\
15940 \\
14165 \\
13082\end{array}$ & $\begin{array}{r}\text { RTYC } \\
17728 \\
18041 \\
17844 \\
18167\end{array}$ & $\begin{array}{l}\text { RPPN } \\
-3887 \\
-2101 \\
-3679 \\
-5085\end{array}$ & $\begin{array}{c}\text { RPMX } \\
13841 \\
15940 \\
14165 \\
13082\end{array}$ \\
\hline $\begin{array}{r}2006 \text { Q1 } \\
\text { Q2 } \\
\text { Q3 } \\
\text { Q4 }\end{array}$ & $\begin{array}{l}40607 \\
39158 \\
42391 \\
43745\end{array}$ & $\begin{array}{l}23413 \\
24844 \\
24848 \\
25157\end{array}$ & $\begin{array}{l}19297 \\
18014 \\
18752 \\
19103\end{array}$ & $\begin{array}{l}42710 \\
42858 \\
43600 \\
44260\end{array}$ & $\begin{array}{r}-2103 \\
-3700 \\
-1209 \\
-515\end{array}$ & $\begin{array}{l}40607 \\
39158 \\
42391 \\
43745\end{array}$ & $\begin{array}{l}17194 \\
14314 \\
17543 \\
18588\end{array}$ & $\begin{array}{l}19297 \\
18014 \\
18752 \\
19103\end{array}$ & $\begin{array}{r}-2103 \\
-3700 \\
-1209 \\
-515\end{array}$ & $\begin{array}{l}17194 \\
14314 \\
17543 \\
18588\end{array}$ \\
\hline $\begin{array}{r}2007 \text { Q1 } \\
\text { Q2 } \\
\text { Q3 } \\
\text { Q4 }\end{array}$ & $\begin{array}{l}44179 \\
41802 \\
42485 \\
41714\end{array}$ & $\begin{array}{l}24418 \\
26569 \\
26432 \\
26319\end{array}$ & $\begin{array}{l}19665 \\
17653 \\
18603 \\
18120\end{array}$ & $\begin{array}{l}44083 \\
44222 \\
45035 \\
44439\end{array}$ & $\begin{array}{r}96 \\
-2420 \\
-2550 \\
-2725\end{array}$ & $\begin{array}{l}44179 \\
41802 \\
42485 \\
41714\end{array}$ & $\begin{array}{l}19761 \\
15233 \\
16053 \\
15395\end{array}$ & $\begin{array}{l}19665 \\
17653 \\
18603 \\
18120\end{array}$ & $\begin{array}{r}96 \\
-2420 \\
-2550 \\
-2725\end{array}$ & $\begin{array}{l}19761 \\
15233 \\
16053 \\
15395\end{array}$ \\
\hline $\begin{array}{r}2008 \text { Q1 } \\
\text { Q2 } \\
\text { Q3 } \\
\text { Q4 }\end{array}$ & $\begin{array}{l}51437 \\
40376 \\
39895 \\
34390\end{array}$ & $\begin{array}{l}26731 \\
28806 \\
26916 \\
29032\end{array}$ & $\begin{array}{l}20771 \\
18603 \\
19679 \\
19608\end{array}$ & $\begin{array}{l}47502 \\
47409 \\
46595 \\
48640\end{array}$ & $\begin{array}{r}3935 \\
-7033 \\
-6700 \\
-14250\end{array}$ & $\begin{array}{l}51437 \\
40376 \\
39895 \\
34390\end{array}$ & $\begin{array}{r}24706 \\
11570 \\
12979 \\
5358\end{array}$ & $\begin{array}{l}20771 \\
18603 \\
19679 \\
19608\end{array}$ & $\begin{array}{r}3935 \\
-7033 \\
-6700 \\
-14250\end{array}$ & $\begin{array}{r}24706 \\
11570 \\
12979 \\
5358\end{array}$ \\
\hline $\begin{array}{r}2009 \text { Q1 } \\
\text { Q2 } \\
\text { Q3 } \\
\text { Q4 }\end{array}$ & $\begin{array}{l}31668^{\dagger} \\
23087 \\
23789 \\
23704\end{array}$ & $\begin{array}{l}28509^{\dagger} \\
30211 \\
31305 \\
31122\end{array}$ & $\begin{array}{l}20266^{\dagger} \\
19315 \\
19529 \\
19663\end{array}$ & $\begin{array}{l}48775^{\dagger} \\
49526 \\
50834 \\
50785\end{array}$ & $\begin{array}{l}-17107^{\dagger} \\
-26439 \\
-27045 \\
-27081\end{array}$ & $\begin{array}{l}31668^{\dagger} \\
23087 \\
23789 \\
23704\end{array}$ & $\begin{array}{r}3159^{\dagger} \\
-7124 \\
-7516 \\
-7418\end{array}$ & $\begin{array}{l}20266^{\dagger} \\
19315 \\
19529 \\
19663\end{array}$ & $\begin{array}{ll}-17 & 107^{\dagger} \\
-26 & 439 \\
-27 & 045 \\
-27 & 081\end{array}$ & $\begin{array}{r}3159^{\dagger} \\
-7124 \\
-7516 \\
-7418\end{array}$ \\
\hline
\end{tabular}




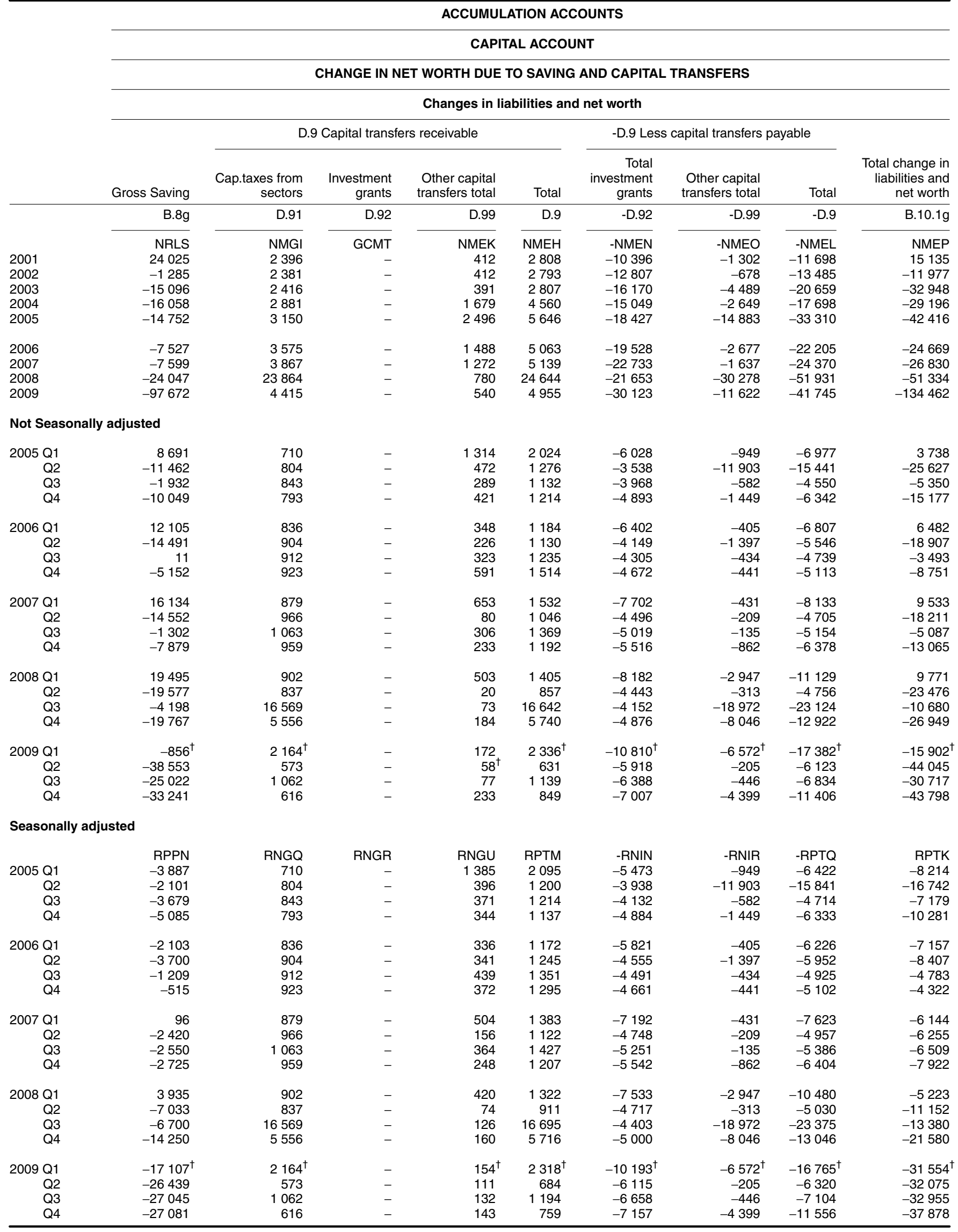




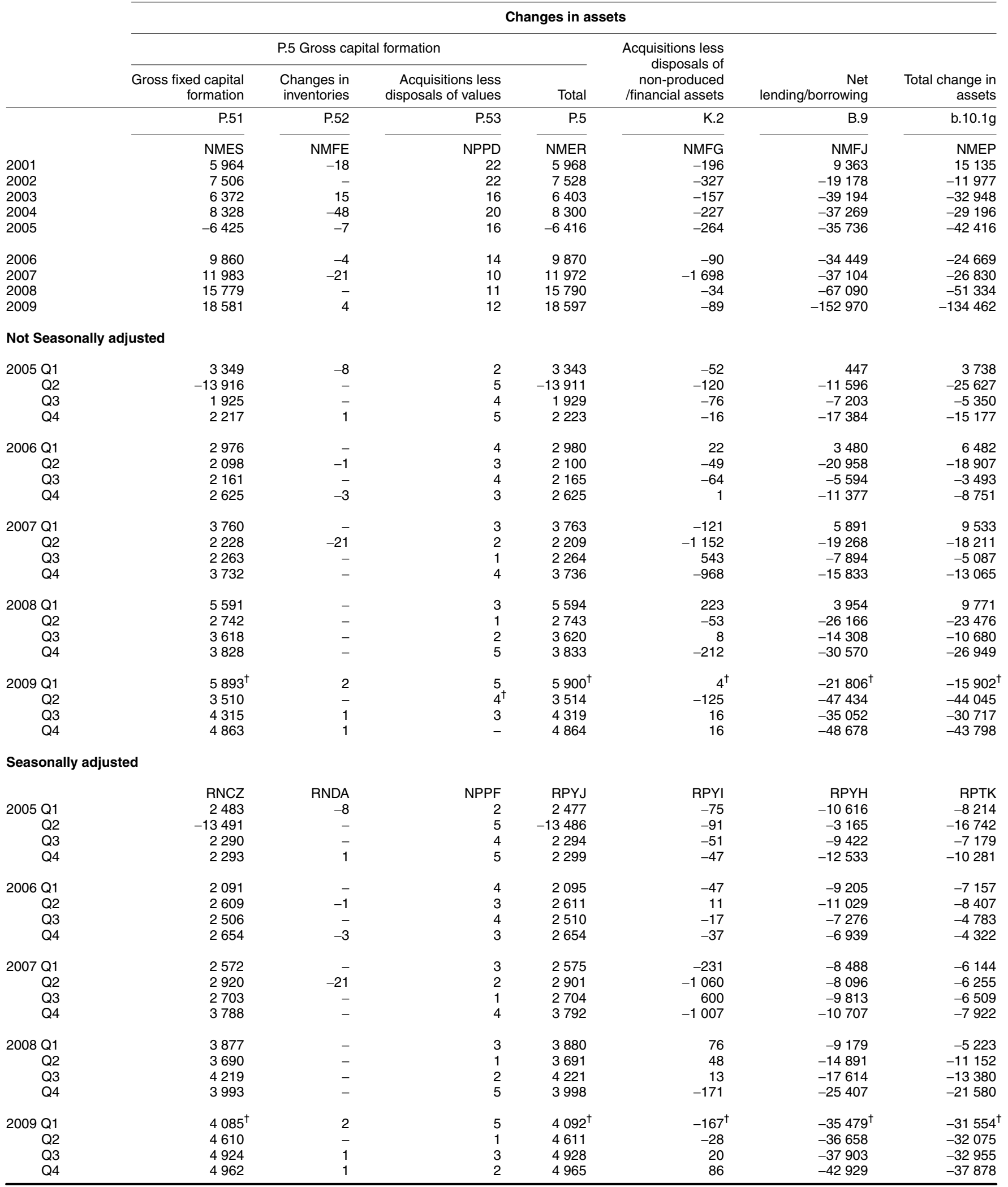




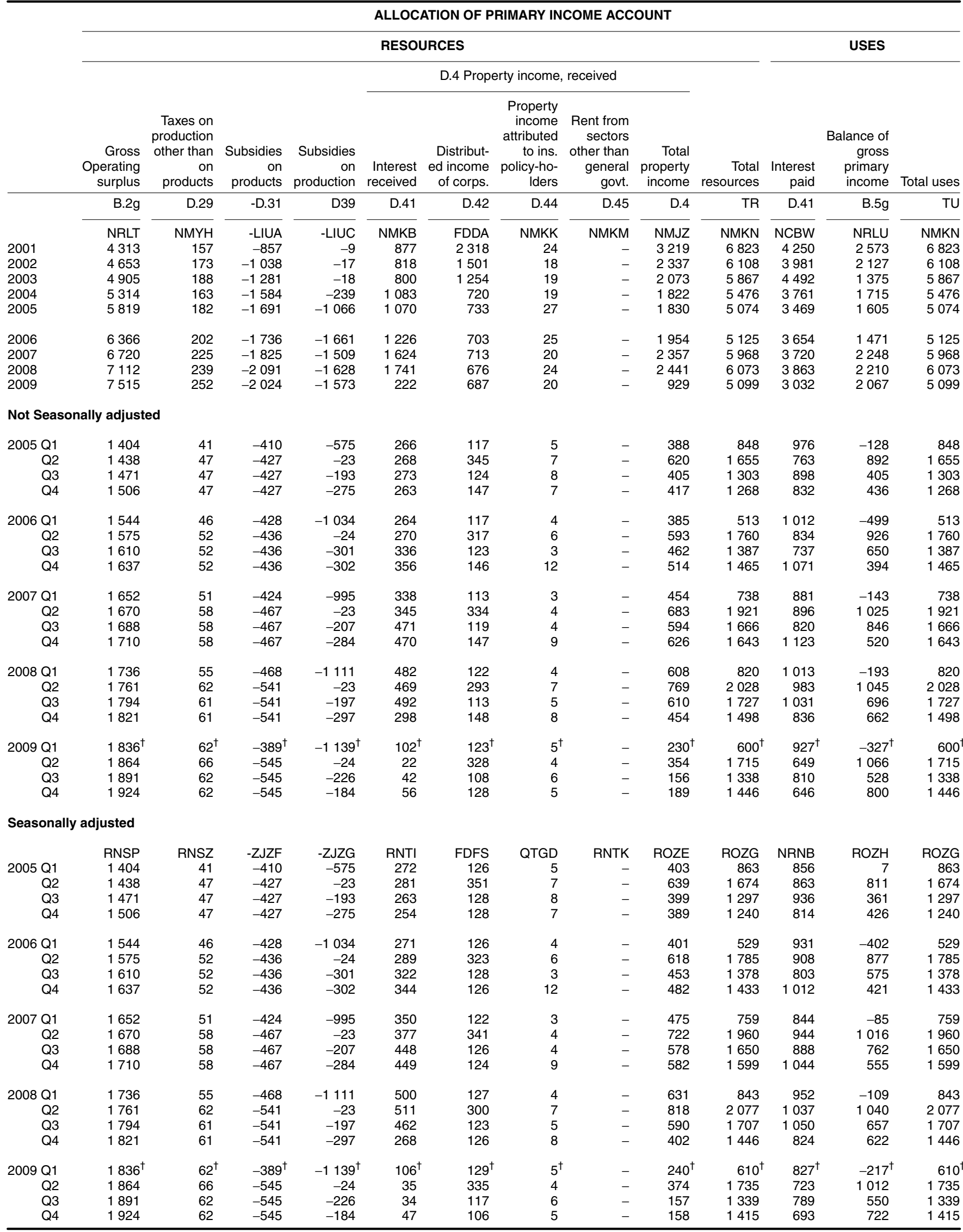




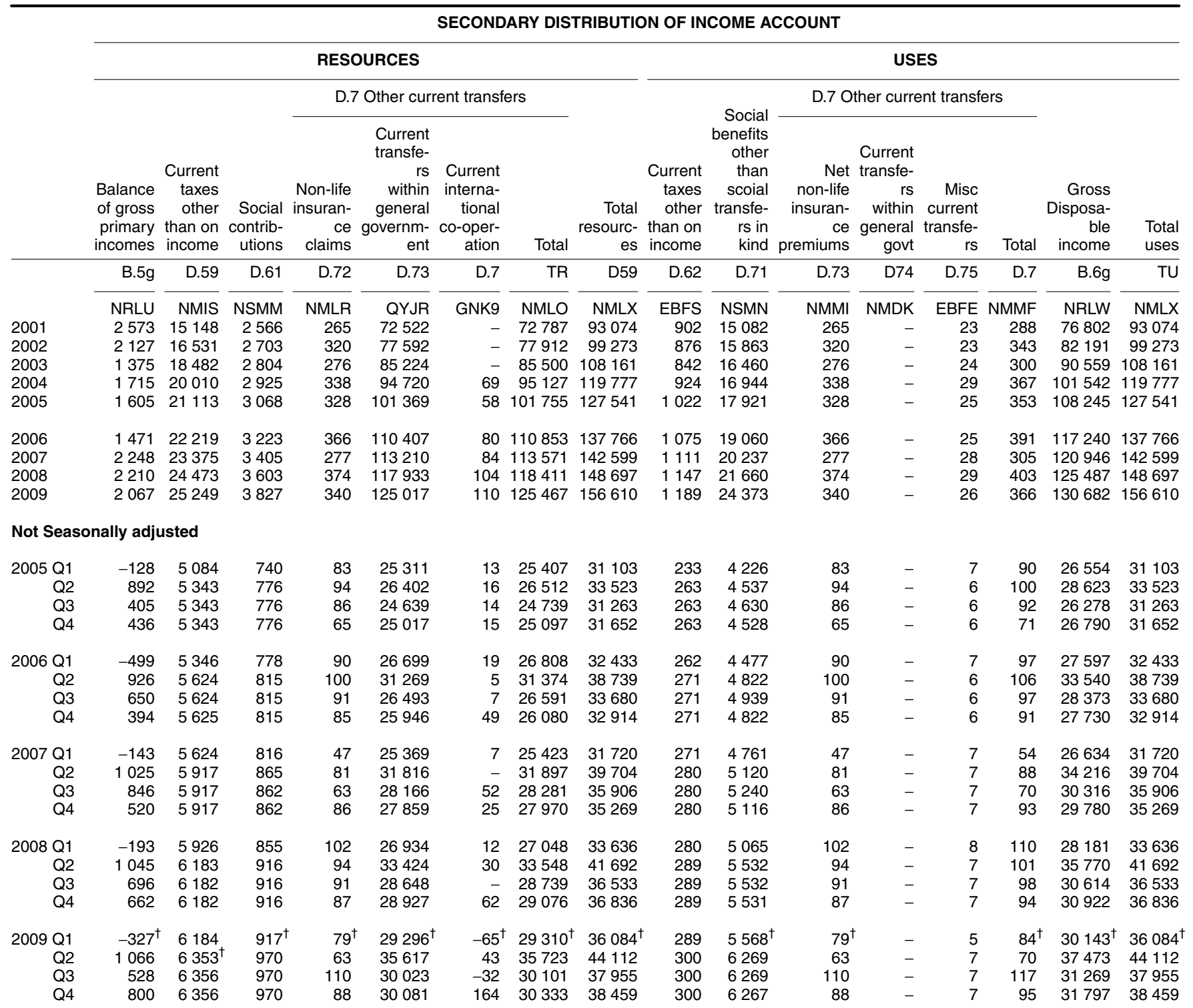

Seasonally adjusted

$\begin{array}{rrrr}2005 \text { Q1 } & \text { ROZH } & \text { RNTO } \\ \text { Q2 } & 811 & 5084 & 740 \\ \text { Q3 } & 361 & 5343 & 776 \\ \text { Q4 } & 426 & 5343 & 776 \\ & & & \\ 2006 \text { Q1 } & -402 & 5346 & 778 \\ \text { Q2 } & 877 & 5624 & 815 \\ \text { Q3 } & 575 & 5624 & 815 \\ \text { Q4 } & 421 & 5625 & 815 \\ & & & \\ 2007 \text { Q1 } & -85 & 5624 & 816 \\ \text { Q2 } & 1016 & 5917 & 8 \\ \text { Q3 } & 762 & 5917 & 8 \\ \text { Q4 } & 555 & 5917 & 8 \\ & & & \\ 2008 \text { Q1 } & -109 & 5926 & 855 \\ \text { Q2 } & 1040 & 6183 & 916 \\ \text { Q3 } & 657 & 6182 & 916 \\ \text { Q4 } & 622 & 6182 & 916 \\ & & & \\ 2009 \text { Q1 } & -217^{\dagger} & 6184 & 917 \\ \text { Q2 } & 1012 & 6353^{\dagger} & 970 \\ \text { Q3 } & 550 & 6356 & 970 \\ \text { Q4 } & 722 & 6356 & 970\end{array}$

$\begin{array}{rrrrrrrr} & \text { RNRV } & \text { RNHL } & \text { IY9B } & \text { RPIU } & \text { RPIV } & \text { EGCC } & \text { RPIX } \\ 740 & 84 & 25684 & 13 & 25781 & 31612 & 233 & 4226 \\ 776 & 90 & 24410 & 16 & 24516 & 31446 & 263 & 4537 \\ 776 & 85 & 25336 & 14 & 25435 & 31915 & 263 & 4630 \\ 776 & 69 & 25939 & 15 & 26023 & 32568 & 263 & 4528 \\ & & & & & & & \\ 778 & 91 & 27609 & 19 & 27719 & 33441 & 262 & 4477 \\ 815 & 94 & 28432 & 5 & 28531 & 35847 & 271 & 4822 \\ 815 & 92 & 27348 & 7 & 27447 & 34461 & 271 & 4939 \\ 815 & 89 & 27018 & 49 & 27156 & 34017 & 271 & 4822 \\ 816 & 48 & 26666 & 7 & 26721 & 33076 & 271 & 4761 \\ 865 & 76 & 28464 & - & 28540 & 36338 & 280 & 5120 \\ 862 & 65 & 29052 & 52 & 29169 & 36710 & 280 & 5240 \\ 862 & 88 & 29028 & 25 & 29141 & 36475 & 280 & 5116 \\ & & & & & & & \\ 855 & 96 & 28126 & 12 & 28234 & 34906 & 280 & 5065 \\ 916 & 95 & 29766 & 30 & 29891 & 38030 & 289 & 5532 \\ 916 & 94 & 29718 & - & 29812 & 37567 & 289 & 5532 \\ 916 & 89 & 30323 & 62 & 30474 & 38194 & 289 & 5531 \\ & & & & & & & \\ 917^{\dagger} & 76^{\dagger} & 30765^{\dagger} & - & 30841^{\dagger} & 37725^{\dagger} & 289 & 5568^{\dagger} \\ 970 & 65 & 31409 & 16 & 31490 & 39825 & 300 & 6269 \\ 970 & 110 & 31247 & - & 31357 & 39233 & 300 & 6269 \\ 970 & 89 & 31596 & 94 & 31779 & 39827 & 300 & 6267\end{array}$

RNPV RNGD EGCF RPIZ RPIW RPIV

$\begin{array}{rrrrrr}84 & - & 7 & 91 & 27062 & 31612 \\ 90 & - & 6 & 96 & 26550 & 31446 \\ 85 & - & 6 & 91 & 26931 & 31915 \\ 69 & - & 6 & 75 & 27702 & 32568 \\ & & & & & \\ 91 & - & 7 & 98 & 28604 & 33441 \\ 94 & - & 6 & 100 & 30654 & 35847 \\ 92 & - & 6 & 98 & 29153 & 34461 \\ 89 & - & 6 & 95 & 28829 & 34017 \\ & & & & & \\ 48 & - & 7 & 55 & 27989 & 33076 \\ 76 & - & 7 & 83 & 30855 & 36338 \\ 65 & - & 7 & 72 & 31118 & 36710 \\ 88 & - & 7 & 95 & 30984 & 36475 \\ & & & & & \\ 96 & - & 8 & 104 & 29457 & 34906 \\ 95 & - & 7 & 102 & 32107 & 38030 \\ 94 & - & 7 & 101 & 31645 & 37567 \\ 89 & - & 7 & 96 & 32278 & 38194 \\ & & & & & \\ 76 & - & 5 & 81^{\dagger} & 31787^{\dagger} & 37725^{\dagger} \\ 65 & - & 7 & 72 & 33184 & 39825 \\ 110 & - & 7 & 117 & 32547 & 39233 \\ 89 & - & 7 & 96 & 33164 & 39827\end{array}$




\begin{tabular}{|c|c|c|c|c|}
\hline & \multicolumn{4}{|c|}{ REDISTRIBUTION OF INCOME IN KIND } \\
\hline & \multirow{2}{*}{$\begin{array}{c}\text { RESOURCES } \\
\text { Total resources, gross } \\
\text { disposable income }\end{array}$} & \multicolumn{3}{|c|}{ USES } \\
\hline & & $\begin{array}{r}\text { Social assistance benefits in } \\
\text { kind }\end{array}$ & $\begin{array}{r}\text { Adjusted gross disposable } \\
\text { income }\end{array}$ & Total uses \\
\hline & B.6g & D.6313 & B.7g & TU \\
\hline & NRLW & NMMU & NSXL & NRLW \\
\hline 2001 & 76802 & 50247 & 26555 & 76802 \\
\hline 2002 & 82191 & 55408 & 26783 & 82191 \\
\hline 2003 & 90559 & 60739 & 29820 & 90559 \\
\hline 2004 & 101542 & 65418 & 36124 & 101542 \\
\hline 2005 & 108245 & 70650 & 37595 & 108245 \\
\hline 2006 & 117240 & 74594 & 42646 & 117240 \\
\hline 2007 & 120946 & 78297 & 42649 & 120946 \\
\hline 2008 & 125487 & 82976 & 42511 & 125487 \\
\hline 2009 & 130682 & 85461 & 45221 & 130682 \\
\hline \multicolumn{5}{|c|}{ Not Seasonally adjusted } \\
\hline 2005 Q1 & 26554 & 16812 & 9742 & 26554 \\
\hline Q2 & 28623 & 17926 & 10697 & 28623 \\
\hline Q3 & 26278 & 17916 & 8362 & 26278 \\
\hline Q4 & 26790 & 17996 & 8794 & 26790 \\
\hline 2006 Q1 & 27597 & 18044 & 9553 & 27597 \\
\hline Q2 & 33540 & 18841 & 14699 & 33540 \\
\hline Q3 & 28373 & 18835 & 9538 & 28373 \\
\hline Q4 & 27730 & 18874 & 8856 & 27730 \\
\hline 2007 Q1 & 26634 & 18918 & 7716 & 26634 \\
\hline Q2 & 34216 & 19784 & 14432 & 34216 \\
\hline Q3 & 30316 & 19746 & 10570 & 30316 \\
\hline Q4 & 29780 & 19849 & 9931 & 29780 \\
\hline 2008 Q1 & 28181 & 20004 & 8177 & 28181 \\
\hline Q2 & 35770 & 20984 & 14786 & 35770 \\
\hline Q3 & 30614 & 20970 & 9644 & 30614 \\
\hline Q4 & 30922 & 21018 & 9904 & 30922 \\
\hline 2009 Q1 & $30143^{\dagger}$ & $20927^{\dagger}$ & $9216^{\dagger}$ & $30143^{\dagger}$ \\
\hline Q2 & 37473 & 21450 & 16023 & 37473 \\
\hline Q3 & 31269 & 21461 & 9808 & 31269 \\
\hline Q4 & 31797 & 21623 & 10174 & 31797 \\
\hline \multicolumn{5}{|c|}{ Seasonally adjusted } \\
\hline & RPIW & QYKJ & RPOC & RPIW \\
\hline 2005 Q1 & 27062 & 17072 & 9990 & 27062 \\
\hline Q2 & 26550 & 17600 & 8950 & 26550 \\
\hline Q3 & 26931 & 17862 & 9069 & 26931 \\
\hline Q4 & 27702 & 18116 & 9586 & 27702 \\
\hline 2006 Q1 & 28604 & 18324 & 10280 & 28604 \\
\hline Q2 & 30654 & 18502 & 12152 & 30654 \\
\hline Q3 & 29153 & 18779 & 10374 & 29153 \\
\hline Q4 & 28829 & 18989 & 9840 & 28829 \\
\hline 2007 Q1 & 27989 & 19209 & 8780 & 27989 \\
\hline Q2 & 30855 & 19440 & 11415 & 30855 \\
\hline Q3 & 31118 & 19692 & 11426 & 31118 \\
\hline Q4 & 30984 & 19956 & 11028 & 30984 \\
\hline 2008 Q1 & 29457 & 20272 & 9185 & 29457 \\
\hline Q2 & 32107 & 20690 & 11417 & 32107 \\
\hline Q3 & 31645 & 20928 & 10717 & 31645 \\
\hline Q4 & 32278 & 21086 & 11192 & 32278 \\
\hline 2009 Q1 & $31787^{\dagger}$ & $21179^{\dagger}$ & $10608^{\dagger}$ & $31787^{\dagger}$ \\
\hline Q2 & 33184 & 21158 & 12026 & 33184 \\
\hline Q3 & 32547 & 21415 & 11132 & 32547 \\
\hline Q4 & 33164 & 21709 & 11455 & 33164 \\
\hline
\end{tabular}


$£$ million

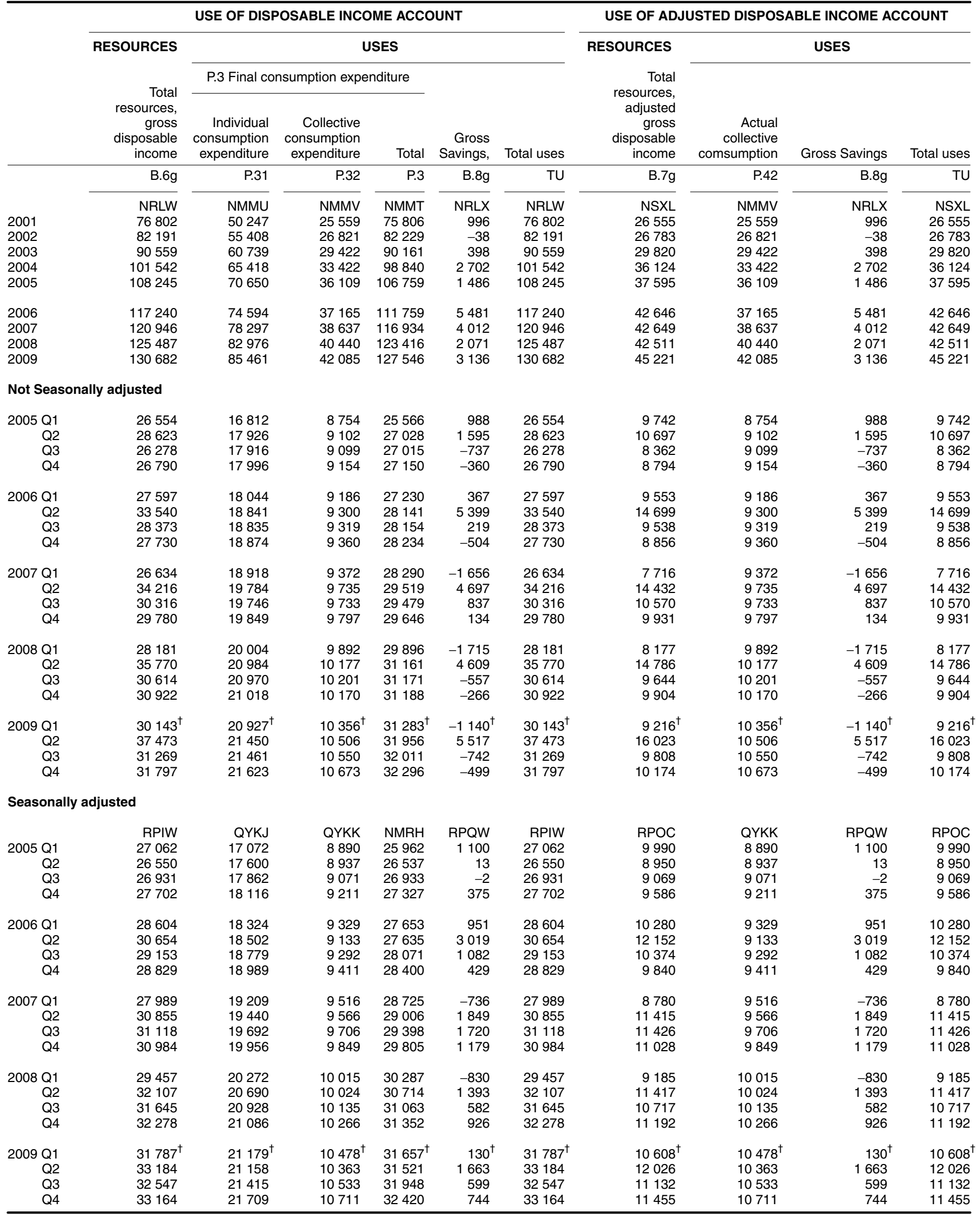




\begin{tabular}{|c|c|c|c|c|c|c|c|c|}
\hline & \multicolumn{8}{|c|}{ ACCUMULATION ACCOUNTS } \\
\hline & \multicolumn{8}{|c|}{ CAPITAL ACCOUNT } \\
\hline & \multicolumn{8}{|c|}{ CHANGE IN NET WORTH DUE TO SAVING AND CAPITAL TRANSFERS } \\
\hline & \multicolumn{8}{|c|}{ Changes in liabilities and net worth } \\
\hline & & \multicolumn{3}{|c|}{ D.9 Capital transfers receivable } & \multicolumn{3}{|c|}{-D.9 Less capital transfers payable } & $\begin{array}{r}\text { Total change in } \\
\text { liabilities and } \\
\text { net worth }\end{array}$ \\
\hline & B.8g & D.92 & D.99 & D.9 & $-\mathrm{D} .92$ & $-\mathrm{D} .99$ & $-\mathrm{D} .9$ & B.10.1g \\
\hline $\begin{array}{l}2001 \\
2002 \\
2003 \\
2004 \\
2005\end{array}$ & $\begin{array}{r}\text { NRLX } \\
996 \\
-38 \\
398 \\
2702 \\
1486\end{array}$ & $\begin{array}{r}\text { NMNE } \\
4652 \\
6328 \\
7360 \\
6804 \\
7582\end{array}$ & $\begin{array}{r}\text { NMNH } \\
416 \\
735 \\
4770 \\
2282 \\
2014\end{array}$ & $\begin{array}{r}\text { NMMY } \\
5068 \\
7063 \\
12130 \\
9086 \\
9596\end{array}$ & $\begin{array}{r}-N M N R \\
-917 \\
-839 \\
-1165 \\
-1127 \\
-1563\end{array}$ & $\begin{array}{r}-N M N U \\
- \\
-5 \\
-5 \\
-1247 \\
-1325\end{array}$ & $\begin{array}{r}-N M N L \\
-917 \\
-844 \\
-1170 \\
-2374 \\
-2888\end{array}$ & $\begin{array}{r}\text { NRMJ } \\
5147 \\
6181 \\
11358 \\
9414 \\
8194\end{array}$ \\
\hline $\begin{array}{l}2006 \\
2007 \\
2008 \\
2009\end{array}$ & $\begin{array}{l}5481 \\
4012 \\
2071 \\
3136\end{array}$ & $\begin{array}{r}8515 \\
9901 \\
10892 \\
12913\end{array}$ & $\begin{array}{l}1110 \\
1684 \\
3998 \\
1426\end{array}$ & $\begin{array}{r}9625 \\
11585 \\
14890 \\
14339\end{array}$ & $\begin{array}{l}-1635 \\
-1859 \\
-1687 \\
-3048\end{array}$ & $\begin{array}{r}-884 \\
-950 \\
-2210 \\
-93\end{array}$ & $\begin{array}{l}-2519 \\
-2809 \\
-3897 \\
-3141\end{array}$ & $\begin{array}{l}12587 \\
12788 \\
13064 \\
14334\end{array}$ \\
\hline \multicolumn{9}{|c|}{ Not Seasonally adjusted } \\
\hline $\begin{array}{r}2005 \text { Q1 } \\
\text { Q2 } \\
\text { Q3 } \\
\text { Q4 }\end{array}$ & $\begin{array}{r}988 \\
1595 \\
-737 \\
-360\end{array}$ & $\begin{array}{l}2326 \\
1437 \\
1636 \\
2183\end{array}$ & $\begin{array}{l}811 \\
282 \\
596 \\
325\end{array}$ & $\begin{array}{l}3137 \\
1719 \\
2232 \\
2508\end{array}$ & $\begin{array}{l}-689 \\
-269 \\
-279 \\
-326\end{array}$ & $\begin{array}{l}-516 \\
-300 \\
-273 \\
-236\end{array}$ & $\begin{array}{r}-1205 \\
-569 \\
-552 \\
-562\end{array}$ & $\begin{array}{r}2920 \\
2745 \\
943 \\
1586\end{array}$ \\
\hline $\begin{array}{r}2006 \text { Q1 } \\
\text { Q2 } \\
\text { Q3 } \\
\text { Q4 }\end{array}$ & $\begin{array}{r}367 \\
5399 \\
219 \\
-504\end{array}$ & $\begin{array}{l}2633 \\
1951 \\
2022 \\
1909\end{array}$ & $\begin{array}{l}300 \\
271 \\
269 \\
270\end{array}$ & $\begin{array}{l}2933 \\
2222 \\
2291 \\
2179\end{array}$ & $\begin{array}{l}-526 \\
-240 \\
-502 \\
-367\end{array}$ & $\begin{array}{l}-224 \\
-210 \\
-202 \\
-248\end{array}$ & $\begin{array}{l}-750 \\
-450 \\
-704 \\
-615\end{array}$ & $\begin{array}{l}2550 \\
7171 \\
1806 \\
1060\end{array}$ \\
\hline $\begin{array}{r}2007 \text { Q1 } \\
\text { Q2 } \\
\text { Q3 } \\
\text { Q4 }\end{array}$ & $\begin{array}{r}-1656 \\
4697 \\
837 \\
134\end{array}$ & $\begin{array}{l}2545 \\
2370 \\
2421 \\
2565\end{array}$ & $\begin{array}{l}247 \\
287 \\
209 \\
941\end{array}$ & $\begin{array}{l}2792 \\
2657 \\
2630 \\
3506\end{array}$ & $\begin{array}{l}-852 \\
-262 \\
-407 \\
-338\end{array}$ & $\begin{array}{r}-620 \\
-60 \\
-105 \\
-165\end{array}$ & $\begin{array}{r}-1472 \\
-322 \\
-512 \\
-503\end{array}$ & $\begin{array}{l}-336 \\
7032 \\
2955 \\
3137\end{array}$ \\
\hline $\begin{array}{r}2008 \text { Q1 } \\
\text { Q2 } \\
\text { Q3 } \\
\text { Q4 }\end{array}$ & $\begin{array}{r}-1715 \\
4609 \\
-557 \\
-266\end{array}$ & $\begin{array}{l}3459 \\
2492 \\
2318 \\
2623\end{array}$ & $\begin{array}{r}2844 \\
443 \\
104 \\
607\end{array}$ & $\begin{array}{l}6303 \\
2935 \\
2422 \\
3230\end{array}$ & $\begin{array}{r}-1242 \\
-129 \\
-154 \\
-162\end{array}$ & $\begin{array}{r}-2097 \\
-10 \\
-57 \\
-46\end{array}$ & $\begin{array}{r}-3339 \\
-139 \\
-211 \\
-208\end{array}$ & $\begin{array}{l}1249 \\
7405 \\
1654 \\
2756\end{array}$ \\
\hline $\begin{array}{r}2009 \text { Q1 } \\
\text { Q2 } \\
\text { Q3 } \\
\text { Q4 }\end{array}$ & $\begin{array}{c}-1140^{\dagger} \\
5517 \\
-742 \\
-499\end{array}$ & $\begin{array}{l}3422^{\dagger} \\
3117 \\
3200 \\
3174\end{array}$ & $\begin{array}{l}841^{\dagger} \\
207 \\
171 \\
207\end{array}$ & $\begin{array}{l}4263^{\dagger} \\
3324 \\
3371 \\
3381\end{array}$ & $\begin{array}{c}-1001^{\dagger} \\
-731 \\
-674 \\
-642\end{array}$ & $\begin{array}{l}-51 \\
2^{\dagger} \\
-16 \\
-28\end{array}$ & $\begin{array}{c}-1052^{\dagger} \\
-729 \\
-690 \\
-670\end{array}$ & $\begin{array}{l}2071^{\dagger} \\
8112 \\
1939 \\
2212\end{array}$ \\
\hline \multicolumn{9}{|c|}{ Seasonally adjusted } \\
\hline $\begin{array}{r}2005 \text { Q1 } \\
\text { Q2 } \\
\text { Q3 } \\
\text { Q4 }\end{array}$ & $\begin{array}{r}\text { RPQW } \\
1100 \\
13 \\
-2 \\
375\end{array}$ & $\begin{array}{l}\text { RNTY } \\
1890 \\
1656 \\
1776 \\
2260\end{array}$ & $\begin{array}{r}\text { RNUE } \\
811 \\
282 \\
596 \\
325\end{array}$ & $\begin{array}{r}\text { RPWF } \\
2701 \\
1938 \\
2372 \\
2585\end{array}$ & $\begin{array}{r}-R N U Z \\
-689 \\
-269 \\
-279 \\
-326\end{array}$ & $\begin{array}{r}-R N V D \\
-516 \\
-300 \\
-273 \\
-236\end{array}$ & $\begin{array}{r}- \text { RPWJ } \\
-1205 \\
-569 \\
-552 \\
-562\end{array}$ & $\begin{array}{r}\text { RPWD } \\
2596 \\
1382 \\
1818 \\
2398\end{array}$ \\
\hline $\begin{array}{r}2006 \text { Q1 } \\
\text { Q2 } \\
\text { Q3 } \\
\text { Q4 }\end{array}$ & $\begin{array}{r}951 \\
3019 \\
1082 \\
429\end{array}$ & $\begin{array}{l}2182 \\
2190 \\
2205 \\
1938\end{array}$ & $\begin{array}{l}300 \\
271 \\
269 \\
270\end{array}$ & $\begin{array}{l}2482 \\
2461 \\
2474 \\
2208\end{array}$ & $\begin{array}{l}-526 \\
-240 \\
-502 \\
-367\end{array}$ & $\begin{array}{l}-224 \\
-210 \\
-202 \\
-248\end{array}$ & $\begin{array}{l}-750 \\
-450 \\
-704 \\
-615\end{array}$ & $\begin{array}{l}2683 \\
5030 \\
2852 \\
2022\end{array}$ \\
\hline $\begin{array}{r}2007 \text { Q1 } \\
\text { Q2 } \\
\text { Q3 } \\
\text { Q4 }\end{array}$ & $\begin{array}{l}-736 \\
1849 \\
1720 \\
1179\end{array}$ & $\begin{array}{l}2097 \\
2570 \\
2640 \\
2594\end{array}$ & $\begin{array}{l}247 \\
287 \\
209 \\
941\end{array}$ & $\begin{array}{l}2344 \\
2857 \\
2849 \\
3535\end{array}$ & $\begin{array}{l}-852 \\
-262 \\
-407 \\
-338\end{array}$ & $\begin{array}{r}-620 \\
-60 \\
-105 \\
-165\end{array}$ & $\begin{array}{r}-1472 \\
-322 \\
-512 \\
-503\end{array}$ & $\begin{array}{r}136 \\
4384 \\
4057 \\
4211\end{array}$ \\
\hline $\begin{array}{r}2008 \text { Q1 } \\
\text { Q2 } \\
\text { Q3 } \\
\text { Q4 }\end{array}$ & $\begin{array}{r}-830 \\
1393 \\
582 \\
926\end{array}$ & $\begin{array}{l}2887 \\
2705 \\
2552 \\
2748\end{array}$ & $\begin{array}{r}2844 \\
443 \\
104 \\
607\end{array}$ & $\begin{array}{l}5731 \\
3148 \\
2656 \\
3355\end{array}$ & $\begin{array}{r}-1242 \\
-129 \\
-154 \\
-162\end{array}$ & $\begin{array}{r}-2097 \\
-10 \\
-57 \\
-46\end{array}$ & $\begin{array}{r}-3339 \\
-139 \\
-211 \\
-208\end{array}$ & $\begin{array}{l}1562 \\
4402 \\
3027 \\
4073\end{array}$ \\
\hline $\begin{array}{r}2009 \text { Q1 } \\
\text { Q2 } \\
\text { Q3 } \\
\text { Q4 }\end{array}$ & $\begin{array}{c}130^{\dagger} \\
1663 \\
599 \\
744\end{array}$ & $\begin{array}{l}2835^{\dagger} \\
3264 \\
3463 \\
3351\end{array}$ & $\begin{array}{l}841^{\dagger} \\
207 \\
171 \\
207\end{array}$ & $\begin{array}{l}3676^{\dagger} \\
3471 \\
3634 \\
3558\end{array}$ & $\begin{array}{c}-1001^{\dagger} \\
-731 \\
-674 \\
-642\end{array}$ & $\begin{array}{r}-51 \\
2^{\dagger} \\
-16 \\
-28\end{array}$ & $\begin{array}{c}-1052^{\dagger} \\
-729 \\
-690 \\
-670\end{array}$ & $\begin{array}{l}2754^{\dagger} \\
4405 \\
3543 \\
3632\end{array}$ \\
\hline
\end{tabular}




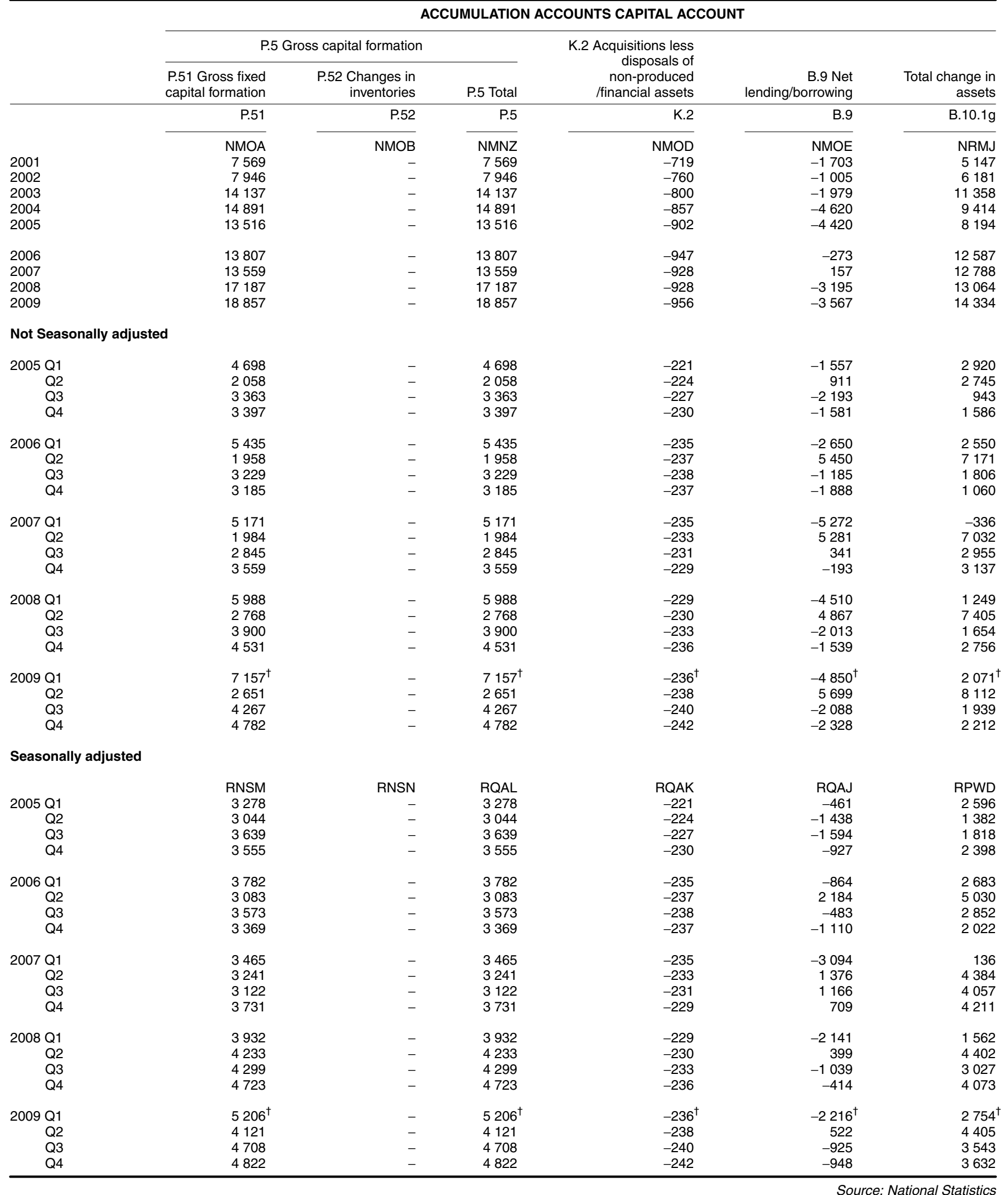


ALLOCATION OF PRIMARY INCOME ACCOUNT

RESOURCES

USES

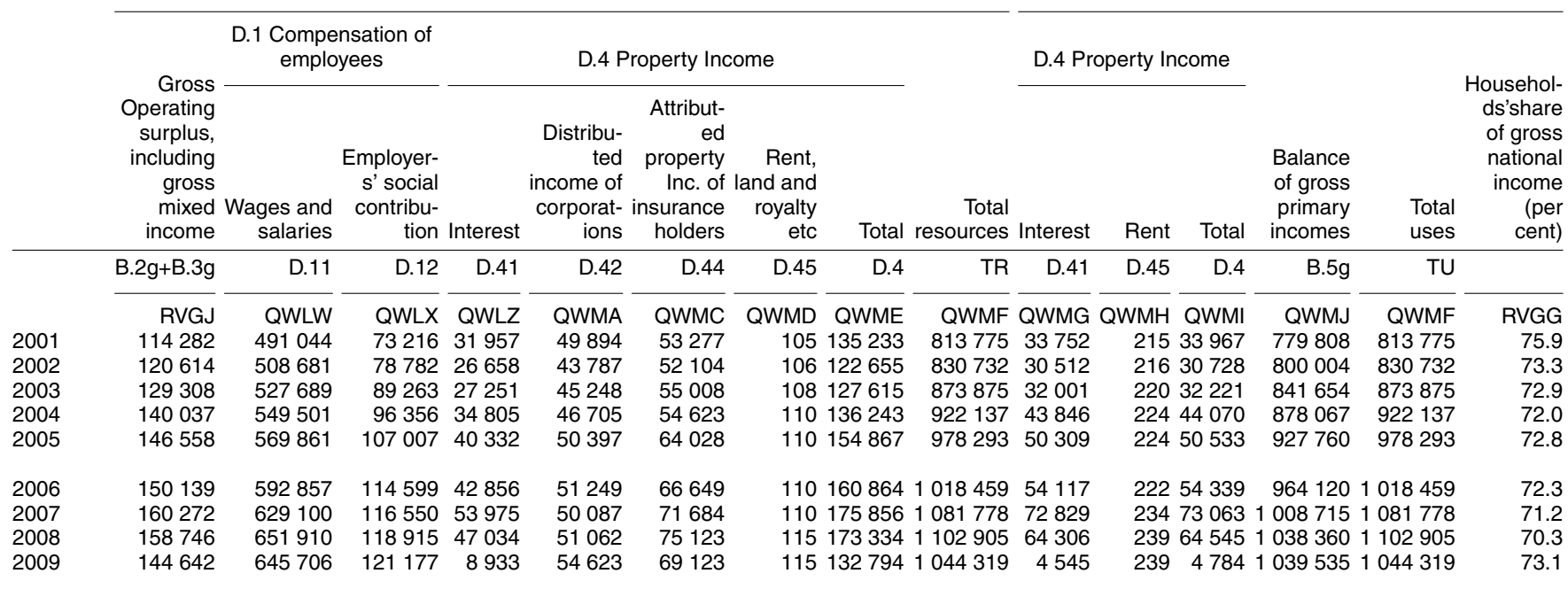

Not Seasonally adjusted

\begin{tabular}{|c|c|c|c|c|c|c|}
\hline 2005 Q1 & 36269 & 146070 & 26824 & 9789 & 10937 & 15524 \\
\hline Q2 & 36517 & 140651 & 25017 & 10055 & 13062 & 16824 \\
\hline Q3 & 36698 & 140199 & 26042 & 10364 & 12964 & 16671 \\
\hline Q4 & 37074 & 142941 & 29124 & 10124 & 13434 & 15009 \\
\hline 2006 Q1 & 37219 & 150499 & 31464 & 10046 & 10549 & 16408 \\
\hline Q2 & 37121 & 147308 & 25741 & 10058 & 14100 & 17242 \\
\hline Q3 & 37622 & 146190 & 26769 & 10913 & 13477 & 17002 \\
\hline Q4 & 38177 & 148860 & 30625 & 11839 & 13123 & 15997 \\
\hline 007 Q1 & 9536 & 159116 & 31155 & 12411 & 11317 & 170 \\
\hline Q2 & 9669 & 154368 & 27914 & 13261 & 14021 & 17 \\
\hline Q3 & 40419 & 155955 & 27872 & 14169 & 12015 & 18 \\
\hline Q4 & 40648 & 159661 & 29609 & 14134 & 12734 & 182 \\
\hline 2008 Q1 & 450 & & 32693 & $13 c$ & & 1854 \\
\hline Q2 & 40600 & 161580 & 28547 & 12662 & 12339 & 2032 \\
\hline Q3 & 40535 & 160687 & 28496 & 12538 & 11912 & 18128 \\
\hline Q4 & 37161 & 161442 & 29179 & 8774 & 15568 & 1813 \\
\hline D09 Q1 & $35416^{\dagger}$ & $166057^{\dagger}$ & $32636^{\dagger}$ & $2957^{\dagger}$ & $13999^{\dagger}$ & \\
\hline Q2 & 35258 & 160649 & 28818 & 1199 & 14480 & \\
\hline Q3 & 36571 & 158552 & 28885 & 2172 & 12559 & \\
\hline Q4 & 37397 & 160448 & 30838 & 2605 & 13585 & 17004 \\
\hline
\end{tabular}

$\begin{array}{llll}28 & 36278 & 245441 & 12599 \\ 28 & 39969 & 242154 & 12467 \\ 28 & 40027 & 242966 & 12563 \\ 26 & 38593 & 247732 & 12680 \\ & & & \\ 28 & 37031 & 256213 & 12737 \\ 28 & 41428 & 251598 & 12597 \\ 28 & 41420 & 252001 & 13760 \\ 26 & 40985 & 258647 & 15023 \\ & & & \\ 28 & 40819 & 270626 & 16962 \\ 28 & 45309 & 267260 & 17361 \\ 28 & 44606 & 268852 & 19273 \\ 26 & 45122 & 275040 & 19233 \\ & & & \\ 28 & 42872 & 284216 & 18379 \\ 29 & 45353 & 276080 & 17242 \\ 29 & 42607 & 272325 & 17322 \\ 29 & 42502 & 270284 & 11363 \\ & & & \\ 29 & 31898^{\dagger} & 266007^{\dagger} & 2789 \\ 29 & 34942 & 259667 & 557 \\ 29 & 34642 & 258650 & 680 \\ 28 & 31312 & 259995 & 519\end{array}$

$5612655 \quad 232786 \quad 245441$

$5612523 \quad 229631 \quad 242154$

$\begin{array}{llll}56 & 12619 & 230347 & 242966\end{array}$

$5612736 \quad 234996 \quad 247732$

$5612793 \quad 243420 \quad 256213$

$\begin{array}{lll}56 & 12653 & 238945 \\ 251598\end{array}$

$5613816 \quad 238185 \quad 252001$

$5415077 \quad 243570 \quad 258647$

$5917021 \quad 253605 \quad 270626$

$5917420 \quad 249840 \quad 267260$

$5919332 \quad 249520 \quad 268852$

$5719290 \quad 255750 \quad 275040$

$\begin{array}{llll}59 & 18438 & 265778 & 284216\end{array}$

$\begin{array}{llll}6017302 & 258778 \quad 276080\end{array}$

$6017382 \quad 254943 \quad 272325$

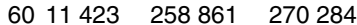

$\begin{array}{llll}60 & 2849^{\dagger} & 263158^{\dagger} & 266007^{\dagger}\end{array}$

$\begin{array}{lllll}60 & 617 & 259050 & 259667\end{array}$

$\begin{array}{llll}60 & 740 & 257910 & 258650\end{array}$

$\begin{array}{llll}59 & 578 & 259417 & 259995\end{array}$
74.2

72.8

72.3

71.8

74.2

73.3

71.1

70.6

73.0

72.4

70.5

70.1

70.9

69.2

71.0

73.7

$75.0^{\dagger}$

72.7

70.8

Seasonally adjusted

$\begin{array}{rrrrrrr}2005 \text { Q1 } & \text { NRJN } & \text { ROYJ } & \text { ROYK } & \text { ROYM } & \text { ROYN } & \text { ROYP } \\ \text { Q2 } & 36543 & 141419 & 24898 & 10010 & 12736 & 15542 \\ \text { Q3 } & 36625 & 142771 & 26639 & 10156 & 12101 & 16113 \\ \text { Q4 } & 37116 & 144183 & 28061 & 9840 & 12740 & 15885 \\ & & & & & & \\ 2006 \text { Q1 } & 37236 & 145312 & 29311 & 10398 & 12159 & 16537 \\ \text { Q2 } & 37095 & 148328 & 27506 & 10241 & 13088 & 16522 \\ \text { Q3 } & 37655 & 149002 & 28212 & 10811 & 13554 & 16855 \\ \text { Q4 } & 38153 & 150215 & 29570 & 11406 & 12448 & 16735 \\ & & & & & & \\ 2007 \text { Q1 } & 39155 & 153286 & 29109 & 13064 & 12754 & 17325 \\ \text { Q2 } & 39868 & 155831 & 29680 & 13572 & 13133 & 17176 \\ \text { Q3 } & 40577 & 158908 & 29127 & 13923 & 12298 & 18156 \\ \text { Q4 } & 40672 & 161075 & 28634 & 13416 & 11902 & 19027 \\ & & & & & & \\ 2008 \text { Q1 } & 40515 & 162503 & 30303 & 13739 & 12128 & 18998 \\ \text { Q2 } & 40571 & 162934 & 29925 & 13299 & 11492 & 19357 \\ \text { Q3 } & 40561 & 163657 & 29754 & 12112 & 12516 & 17856 \\ \text { Q4 } & 37099 & 162816 & 28933 & 7884 & 14926 & 18912 \\ & & & & & & \\ 2009 \text { Q1 } & 35180^{\dagger} & 160979^{\dagger} & 30121^{\dagger} & 3406^{\dagger} & 15438^{\dagger} & 15618^{\dagger} \\ \text { Q2 } & 35563 & 161734 & 30093 & 1538 & 13239 & 18110 \\ \text { Q3 } & 36483 & 161542 & 30227 & 1964 & 13057 & 19169 \\ \text { Q4 } & 37416 & 161451 & 30736 & 2025 & 12889 & 16226\end{array}$

$\begin{array}{rrrr}\text { ROYQ } & \text { ROYL } & \text { ROYR } & \text { ROYU } \\ 28 & 38316 & 240907 & 12558 \\ 28 & 38398 & 243068 & 12755 \\ 28 & 39662 & 246467 & 12445 \\ 26 & 38491 & 247851 & 12551 \\ & & & \\ 28 & 39122 & 250981 & 12690 \\ 28 & 39879 & 252808 & 12941 \\ 28 & 41248 & 256117 & 13610 \\ 26 & 40615 & 258553 & 14876 \\ & & & \\ 28 & 43171 & 264721 & 16903 \\ 28 & 43909 & 269288 & 17811 \\ 28 & 44405 & 273017 & 19060 \\ 26 & 44371 & 274752 & 19055 \\ & & & \\ 28 & 44893 & 278214 & 18588 \\ 29 & 44177 & 277607 & 17858 \\ 29 & 42513 & 276485 & 17000 \\ 29 & 41751 & 270599 & 10860 \\ & & & \\ 29 & 34491^{\dagger} & 260771^{\dagger} & 3026^{\dagger} \\ 29 & 32916 & 260306 & 1072 \\ 29 & 34219 & 262471 & 333 \\ 28 & 31168 & 260771 & 114\end{array}$

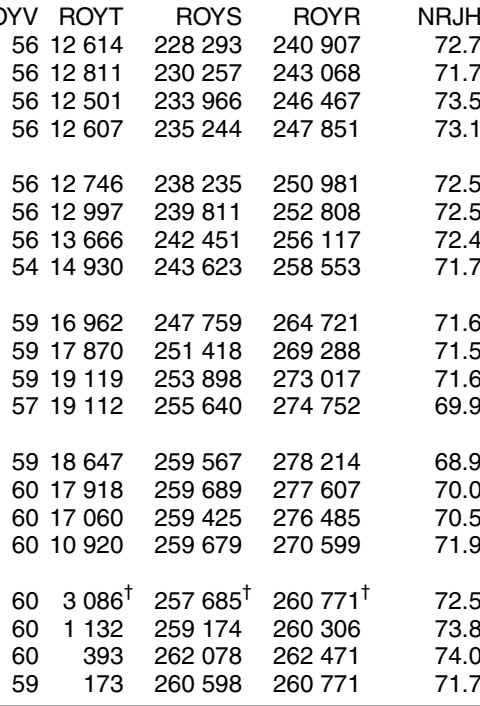




\section{$14.8 \mathrm{~B}$}

$£$ million

\begin{tabular}{|c|c|c|c|c|c|c|c|}
\hline & \multicolumn{7}{|c|}{ SECONDARY DISTRIBUTION OF INCOME ACCOUNT } \\
\hline & \multirow[b]{2}{*}{$\begin{array}{l}\text { Balance of gross } \\
\text { primary incomes }\end{array}$} & \multirow[b]{2}{*}{$\begin{array}{r}\text { Imputed social } \\
\text { contributions }\end{array}$} & \multicolumn{2}{|c|}{ RESOURCES } & & & \multirow[b]{2}{*}{ Total resources } \\
\hline & & & $\begin{array}{r}\text { Social benefit } \\
\text { other than transfer } \\
\text { in kind }\end{array}$ & $\begin{array}{r}\text { Non-life insurance } \\
\text { claims }\end{array}$ & $\begin{array}{r}\text { Miscellaneous } \\
\text { current transfers }\end{array}$ & Total & \\
\hline & B.5g & D.612 & D.62 & D.72 & D.75 & D.7 & TR \\
\hline & QWMJ & RVFH & QWML & QWMM & QWMN & QWMO & QWMP \\
\hline 2001 & 779808 & 502 & 171814 & 11723 & 29080 & 40803 & 992927 \\
\hline 2002 & 800004 & 530 & 182673 & 17327 & 33041 & 50368 & 1033575 \\
\hline 2003 & 841654 & 505 & 193596 & 13890 & 34687 & 48577 & 1084332 \\
\hline 2004 & 878067 & 499 & 198691 & 17479 & 34845 & 52324 & 1129581 \\
\hline 2005 & 927760 & 506 & 212272 & 17199 & 37840 & 55039 & 1195577 \\
\hline 2006 & 964120 & 514 & 226629 & 20713 & 38729 & 59442 & 1250705 \\
\hline 2007 & 1008715 & 518 & 227520 & 14842 & 40518 & 55360 & 1292113 \\
\hline \multicolumn{8}{|c|}{ Not Seasonally adjusted } \\
\hline 2005 Q1 & 232786 & 125 & 49571 & 4337 & 8997 & 13334 & 295816 \\
\hline Q2 & 229631 & 127 & 51492 & 4921 & 9585 & 14506 & 295756 \\
\hline Q3 & 230347 & 127 & 53458 & 4520 & 9429 & 13949 & 297881 \\
\hline Q4 & 234996 & 127 & 57751 & 3421 & 9829 & 13250 & 306124 \\
\hline 2006 Q1 & 243420 & 127 & 54217 & 5081 & 9001 & 14082 & 311846 \\
\hline Q2 & 238945 & 129 & 55919 & 5663 & 9620 & 15283 & 310276 \\
\hline Q3 & 238185 & 129 & 57724 & 5141 & 10213 & 15354 & 311392 \\
\hline Q4 & 243570 & 129 & 58769 & 4828 & 9895 & 14723 & 317191 \\
\hline 2007 Q1 & 253605 & 129 & 54769 & 2528 & 9478 & 12006 & 320509 \\
\hline Q2 & 249840 & 131 & 55868 & 4331 & 9522 & 13853 & 319692 \\
\hline Q3 & 249520 & 129 & 56973 & 3375 & 10025 & 13400 & 320022 \\
\hline Q4 & 255750 & 129 & 59910 & 4608 & 11493 & 16101 & 331890 \\
\hline 2008 Q1 & 265778 & 131 & 58179 & 5452 & 9704 & 15156 & 339244 \\
\hline Q4 & 258861 & 131 & 66717 & 4677 & 10673 & 15350 & 341059 \\
\hline 2009 Q1 & $263158^{\dagger}$ & 131 & $65026^{\dagger}$ & $4259^{\dagger}$ & $9609^{\dagger}$ & $13868^{\dagger}$ & $342183^{\dagger}$ \\
\hline Q2 & 259050 & 131 & 68314 & 3398 & 10497 & 13895 & 341390 \\
\hline Q3 & 257910 & 131 & 69547 & 5912 & 10547 & 16459 & 344047 \\
\hline Q4 & 259417 & 131 & 72569 & 4714 & 11477 & 16191 & 348308 \\
\hline \multicolumn{8}{|c|}{ Seasonally adjusted } \\
\hline & ROYS & & $\mathrm{RPHL}$ & RPHN & $\mathrm{RPHO}$ & RPHM & RPHP \\
\hline 2005 Q1 & 228293 & 125 & 50832 & 4279 & 8997 & 13276 & 292526 \\
\hline Q2 & 230257 & 127 & 52267 & 4711 & 9585 & 14296 & 296947 \\
\hline Q3 & 233966 & 127 & 53635 & 4506 & 9429 & 13935 & 301663 \\
\hline Q4 & 235244 & 127 & 55538 & 3703 & 9829 & 13532 & 304441 \\
\hline 2006 Q1 & 238235 & 127 & 55570 & 5088 & 9001 & 14089 & 308021 \\
\hline Q2 & 239811 & 129 & 56616 & 5328 & 9620 & 14948 & 311504 \\
\hline Q3 & 242451 & 129 & 57713 & 5201 & 10213 & 15414 & 315707 \\
\hline Q4 & 243623 & 129 & 56730 & 5096 & 9895 & 14991 & 315473 \\
\hline 2007 Q1 & 247759 & 129 & 56110 & 2545 & 9478 & 12023 & 316021 \\
\hline Q2 & 251418 & 131 & 56556 & 4055 & 9522 & 13577 & 321682 \\
\hline Q3 & 253898 & 129 & 56927 & 3488 & 10025 & 13513 & 324467 \\
\hline Q4 & 255640 & 129 & 57927 & 4754 & 11493 & 16247 & 329943 \\
\hline 2008 Q1 & 259567 & 131 & 59486 & 5108 & 9704 & 14812 & 333996 \\
\hline Q2 & 259689 & 131 & 62551 & 5062 & 9697 & 14759 & 337130 \\
\hline Q3 & 259425 & 131 & 63832 & 5012 & 10247 & 15259 & 338647 \\
\hline Q4 & 259679 & 131 & 64468 & 4842 & 10673 & 15515 & 339793 \\
\hline 2009 Q1 & $257685^{\dagger}$ & 131 & $66503^{\dagger}$ & $4097^{\dagger}$ & $9544^{\dagger}$ & $13641^{\dagger}$ & $337960^{\dagger}$ \\
\hline Q2 & 259174 & 131 & 68946 & 3511 & 10524 & 14035 & 342286 \\
\hline Q3 & 262078 & 131 & 69751 & 5940 & 10515 & 16455 & 348415 \\
\hline Q4 & 260598 & 131 & 70256 & 4735 & 11547 & 16282 & 347267 \\
\hline
\end{tabular}




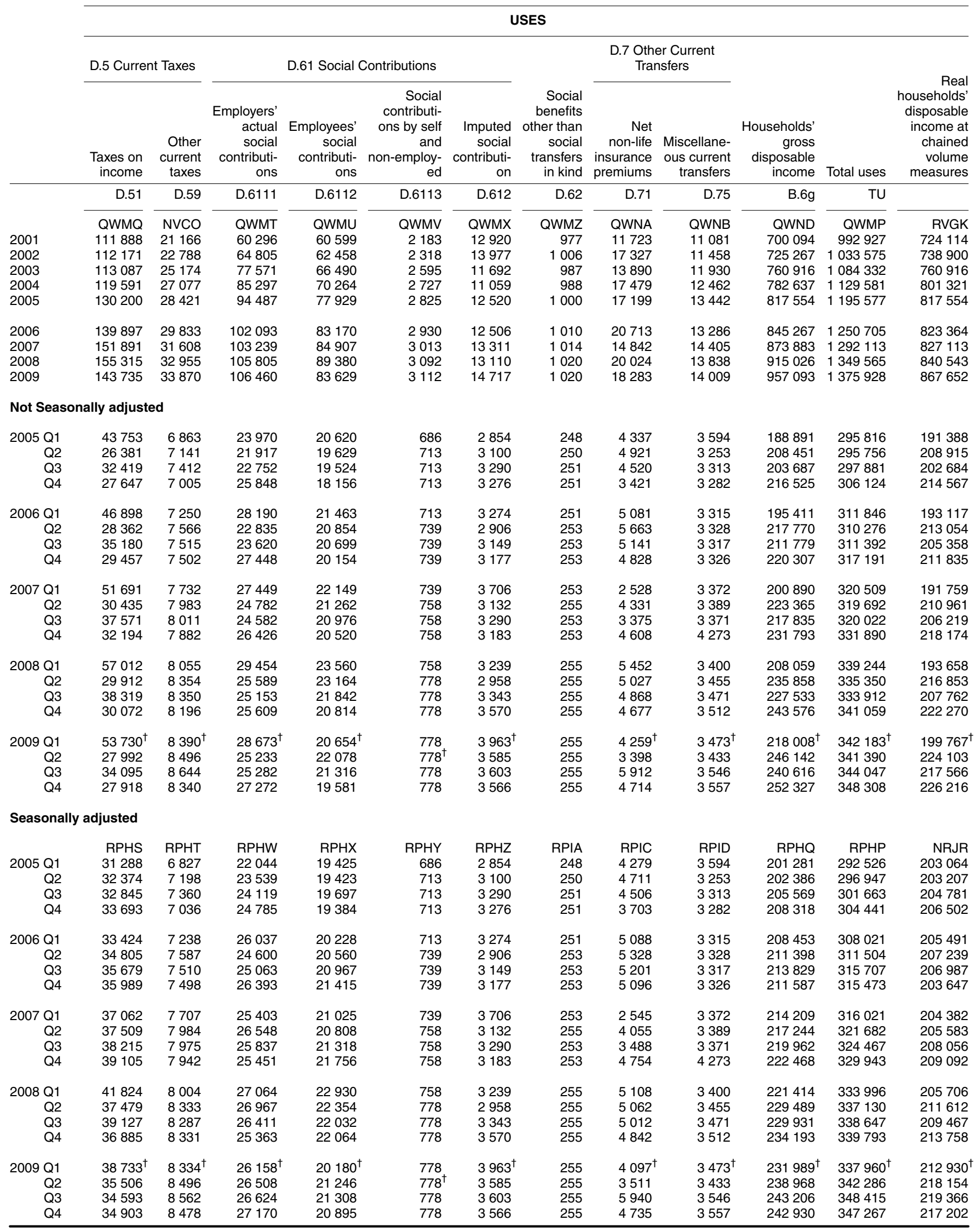




\begin{tabular}{|c|c|c|c|c|c|c|c|c|c|c|}
\hline & \multicolumn{10}{|c|}{ REDISTRIBUTION OF INCOME IN KIND } \\
\hline & \multicolumn{5}{|c|}{ RESOURCES } & \multicolumn{5}{|c|}{ USES } \\
\hline & \multirow[b]{2}{*}{$\begin{array}{r}\text { Households' } \\
\text { gross } \\
\text { disposable } \\
\text { income }\end{array}$} & \multicolumn{3}{|c|}{ D.63 Social transfers in kind } & \multirow[b]{2}{*}{$\begin{array}{r}\text { Total } \\
\text { resources }\end{array}$} & \multicolumn{3}{|c|}{ D.63 Social transfers in kind } & \multirow[b]{2}{*}{$\begin{array}{r}\text { Households' } \\
\text { adjusted } \\
\text { gross } \\
\text { disposable } \\
\text { income }\end{array}$} & \multirow[b]{2}{*}{ Total uses } \\
\hline & & $\begin{array}{r}\text { Social } \\
\text { assistance } \\
\text { benefits in } \\
\text { kind }\end{array}$ & $\begin{array}{r}\text { Transfers of } \\
\text { individual } \\
\text { non-market } \\
\text { goods and } \\
\text { services }\end{array}$ & Total & & $\begin{array}{r}\text { Social } \\
\text { assistance } \\
\text { benefits in } \\
\text { kind }\end{array}$ & $\begin{array}{r}\text { Transfers of } \\
\text { individual } \\
\text { non-market } \\
\text { goods and } \\
\text { services }\end{array}$ & Total & & \\
\hline & B.6g & D.6313 & D.632 & D.63 & TR & D.6313 & D.632 & D.63 & B.7g & TU \\
\hline & QWND & QWNH & NSSA & NSSB & NSSC & HAEJ & HABK & HAEK & NSSD & NSSC \\
\hline 2001 & 700094 & - & 143569 & 143569 & 843663 & - & 25111 & 25111 & 818552 & 843663 \\
\hline 2002 & 725267 & - & 157238 & 157238 & 882505 & - & 26422 & 26422 & 856083 & 882505 \\
\hline 2003 & 760916 & - & 171622 & 171622 & 932538 & - & 27668 & 27668 & 904870 & 932538 \\
\hline 2004 & 782637 & - & 177986 & 177986 & 960623 & - & 29197 & 29197 & 931426 & 960623 \\
\hline 2005 & 817554 & - & 191023 & 191023 & 1008577 & - & 30824 & 30824 & 977753 & 1008577 \\
\hline 2006 & 845267 & - & 205295 & 205295 & 1050562 & - & 32439 & 32439 & 1018123 & 1050562 \\
\hline 2007 & 873883 & - & 215757 & 215757 & 1089640 & - & 33722 & 33722 & 1055918 & 1089640 \\
\hline 2008 & 915026 & - & 231590 & 231590 & 1146616 & - & 37129 & 37129 & 1109487 & 1146616 \\
\hline 2009 & 957093 & - & 241942 & 241942 & 1199035 & - & 35334 & 35334 & 1163701 & 1199035 \\
\hline \multicolumn{11}{|c|}{ Not Seasonally adjusted } \\
\hline 2005 Q1 & 188891 & - & 46186 & 46186 & 235077 & - & 7653 & 7653 & 227424 & 235077 \\
\hline Q2 & 208451 & - & 47739 & 47739 & 256190 & - & 7689 & 7689 & 248501 & 256190 \\
\hline Q3 & 203687 & - & 48301 & 48301 & 251988 & - & 7729 & 7729 & 244259 & 251988 \\
\hline Q4 & 216525 & - & 48797 & 48797 & 265322 & - & 7753 & 7753 & 257569 & 265322 \\
\hline 2006 Q1 & 195411 & - & 49982 & 49982 & 245393 & - & 7913 & 7913 & 237480 & 245393 \\
\hline Q2 & 217770 & - & 51346 & 51346 & 269116 & - & 8048 & 8048 & 261068 & 269116 \\
\hline Q3 & 211779 & - & 51827 & 51827 & 263606 & - & 8172 & 8172 & 255434 & 263606 \\
\hline Q4 & 220307 & - & 52140 & 52140 & 272447 & - & 8306 & 8306 & 264141 & 272447 \\
\hline 2007 Q1 & 200890 & - & 52213 & 52213 & 253103 & - & 8177 & 8177 & 244926 & 253103 \\
\hline Q2 & 223365 & - & 54163 & 54163 & 277528 & - & 8317 & 8317 & 269211 & 277528 \\
\hline Q3 & 217835 & - & 54716 & 54716 & 272551 & - & 8478 & 8478 & 264073 & 272551 \\
\hline Q4 & 231793 & - & 54665 & 54665 & 286458 & - & 8750 & 8750 & 277708 & 286458 \\
\hline 2008 Q1 & 208059 & - & 56777 & 56777 & 264836 & - & 9114 & 9114 & 255722 & 264836 \\
\hline Q2 & 235858 & - & 58529 & 58529 & 294387 & - & 9362 & 9362 & 285025 & 294387 \\
\hline Q3 & 227533 & - & 57137 & 57137 & 284670 & - & 9376 & 9376 & 275294 & 284670 \\
\hline Q4 & 243576 & - & 59147 & 59147 & 302723 & - & 9277 & 9277 & 293446 & 302723 \\
\hline 2009 Q1 & $218008^{\dagger}$ & - & $59362^{\dagger}$ & $59362^{\dagger}$ & $277370^{\dagger}$ & - & $8901^{\dagger}$ & $8901^{\dagger}$ & $268469^{\dagger}$ & $277370^{\dagger}$ \\
\hline Q2 & 246142 & - & 59725 & 59725 & 305867 & - & 8829 & 8829 & 297038 & 305867 \\
\hline Q3 & 240616 & - & 61580 & 61580 & 302196 & - & 8794 & 8794 & 293402 & 302196 \\
\hline Q4 & 252327 & - & 61275 & 61275 & 313602 & - & 8810 & 8810 & 304792 & 313602 \\
\hline \multicolumn{11}{|c|}{ Seasonally adjusted } \\
\hline & $\mathrm{RPHQ}$ & RPNU & RPNV & RPNS & RPNW & RNLQ & RNLS & RPNY & RPNX & RPNW \\
\hline 2005 Q1 & 201281 & - & 45976 & 45976 & 247257 & - & 7653 & 7653 & 239604 & 247257 \\
\hline Q2 & 202386 & - & 47645 & 47645 & 250031 & - & 7689 & 7689 & 242342 & 250031 \\
\hline Q3 & 205569 & - & 48352 & 48352 & 253921 & - & 7729 & 7729 & 246192 & 253921 \\
\hline Q4 & 208318 & - & 49050 & 49050 & 257368 & - & 7753 & 7753 & 249615 & 257368 \\
\hline 2006 Q1 & 208453 & - & 49650 & 49650 & 258103 & - & 7913 & 7913 & 250190 & 258103 \\
\hline Q2 & 211398 & - & 51394 & 51394 & 262792 & - & 8048 & 8048 & 254744 & 262792 \\
\hline Q3 & 213829 & - & 51799 & 51799 & 265628 & - & 8172 & 8172 & 257456 & 265628 \\
\hline Q4 & 211587 & - & 52452 & 52452 & 264039 & - & 8306 & 8306 & 255733 & 264039 \\
\hline 2007 Q1 & 214209 & - & 51804 & 51804 & 266013 & - & 8177 & 8177 & 257836 & 266013 \\
\hline Q2 & 217244 & - & 54326 & 54326 & 271570 & - & 8317 & 8317 & 263253 & 271570 \\
\hline Q3 & 219962 & - & 54602 & 54602 & 274564 & - & 8478 & 8478 & 266086 & 274564 \\
\hline Q4 & 222468 & - & 55025 & 55025 & 277493 & - & 8750 & 8750 & 268743 & 277493 \\
\hline 2008 Q1 & 221414 & - & 56117 & 56117 & 277531 & - & 9114 & 9114 & 268417 & 277531 \\
\hline Q2 & 229489 & - & 58858 & 58858 & 288347 & - & 9362 & 9362 & 278985 & 288347 \\
\hline Q3 & 229931 & - & 57220 & 57220 & 287151 & - & 9376 & 9376 & 277775 & 287151 \\
\hline Q4 & 234193 & - & 59395 & 59395 & 293588 & - & 9277 & 9277 & 284311 & 293588 \\
\hline 2009 Q1 & $231989^{\dagger}$ & - & $58589^{\dagger}$ & $58589^{\dagger}$ & $290578^{\dagger}$ & - & $8901^{\dagger}$ & $8901^{\dagger}$ & $281677^{\dagger}$ & $290578^{\dagger}$ \\
\hline Q2 & 238968 & - & 60198 & 60198 & 299166 & - & 8829 & 8829 & 290337 & 299166 \\
\hline Q3 & 243206 & - & 61514 & 61514 & 304720 & - & 8794 & 8794 & 295926 & 304720 \\
\hline Q4 & 242930 & - & 61641 & 61641 & 304571 & - & 8810 & 8810 & 295761 & 304571 \\
\hline
\end{tabular}


$£$ million

\begin{tabular}{|c|c|c|c|c|c|c|c|c|c|c|c|c|c|}
\hline & \multicolumn{6}{|c|}{ USE OF DISPOSABLE INCOME ACCOUNT } & \multicolumn{7}{|c|}{ USE OF ADJUSTED DISPOSABLE INCOME ACCOUNT } \\
\hline & \multicolumn{3}{|c|}{ RESOURCES } & \multicolumn{3}{|c|}{ USES } & \multicolumn{3}{|c|}{ RESOURCES } & \multicolumn{4}{|c|}{ USES } \\
\hline & $\begin{array}{r}\text { Household- } \\
\text { s' gross } \\
\text { disposable } \\
\text { income }\end{array}$ & $\begin{array}{r}\text { Adj. for } \\
\text { the change } \\
\text { in net } \\
\text { equity of } \\
\text { h'holds in } \\
\text { pen. funds }\end{array}$ & $\begin{array}{r}\text { Total } \\
\text { available } \\
\text { household- } \\
s^{\prime} \\
\text { resources }\end{array}$ & $\begin{array}{r}\text { Final } \\
\text { consumpti- } \\
\text { on } \\
\text { expenditu- } \\
\text { re }\end{array}$ & $\begin{array}{r}\text { Gross } \\
\text { Savings }\end{array}$ & Total uses & $\begin{array}{r}\text { Household- } \\
\mathrm{s}^{\prime} \\
\text { adjusted } \\
\text { gross } \\
\text { disposable } \\
\text { income }\end{array}$ & $\begin{array}{r}\text { Adj. for } \\
\text { the change } \\
\text { in net } \\
\text { equity of } \\
\text { 'holds in } \\
\text { pen. funds }\end{array}$ & $\begin{array}{r}\text { Total } \\
\text { available } \\
\text { household- } \\
s^{\prime} \\
\text { resources }\end{array}$ & $\begin{array}{r}\text { Actual } \\
\text { collective } \\
\text { comsumpti- } \\
\text { on }\end{array}$ & $\begin{array}{r}\text { Gross } \\
\text { Savings }\end{array}$ & Total uses & $\begin{array}{r}\text { Household- } \\
\text { s'saving } \\
\text { ratio(per } \\
\text { cent) }\end{array}$ \\
\hline & B.6g & D.8 & TR & P.31 & B.8g & TU & B.7g & D.8 & TR & P.41 & B.8g & TU & \\
\hline & QWND & NSSE & NSSF & NSSG & NSSH & NSSF & NSSD & NSSE & NSSJ & ABRE & NSSH & NSSJ & RVGL \\
\hline 2001 & 700094 & 16038 & 716132 & 672889 & 43243 & 716132 & 818552 & 16038 & 834590 & 791347 & 43243 & 834590 & 6.0 \\
\hline 2002 & 725267 & 17784 & 743051 & 707386 & 35665 & 743051 & 856083 & 17784 & 873867 & 838202 & 35665 & 873867 & 4.8 \\
\hline 2003 & 760916 & 21377 & 782293 & 742276 & 40017 & 782293 & 904870 & 21377 & 926247 & 886230 & 40017 & 926247 & 5.1 \\
\hline 2004 & 782637 & 6386 & 809023 & 779064 & 29959 & 809023 & 931426 & 26386 & 957812 & 927853 & 29959 & 957812 & 3.7 \\
\hline 2005 & 817554 & 30881 & 848435 & 814964 & 33471 & 848435 & 977753 & 30881 & 1008634 & 975163 & 33471 & 1008634 & 3.9 \\
\hline 2006 & 5267 & 9339 & 874606 & 849475 & & & 1018123 & & 1047462 & 1022331 & 25131 & 1047462 & 2.9 \\
\hline 2008 & 915026 & 27844 & 942870 & 928500 & 14370 & 942870 & 1109487 & 27844 & 1137331 & 1122961 & 14370 & 1137331 & 1.5 \\
\hline 2009 & 957093 & 21555 & 978648 & 910568 & 68080 & 978648 & 1163701 & 21555 & 1185256 & 1117176 & 68080 & 1185256 & 7.0 \\
\hline
\end{tabular}

Not Seasonally adjusted

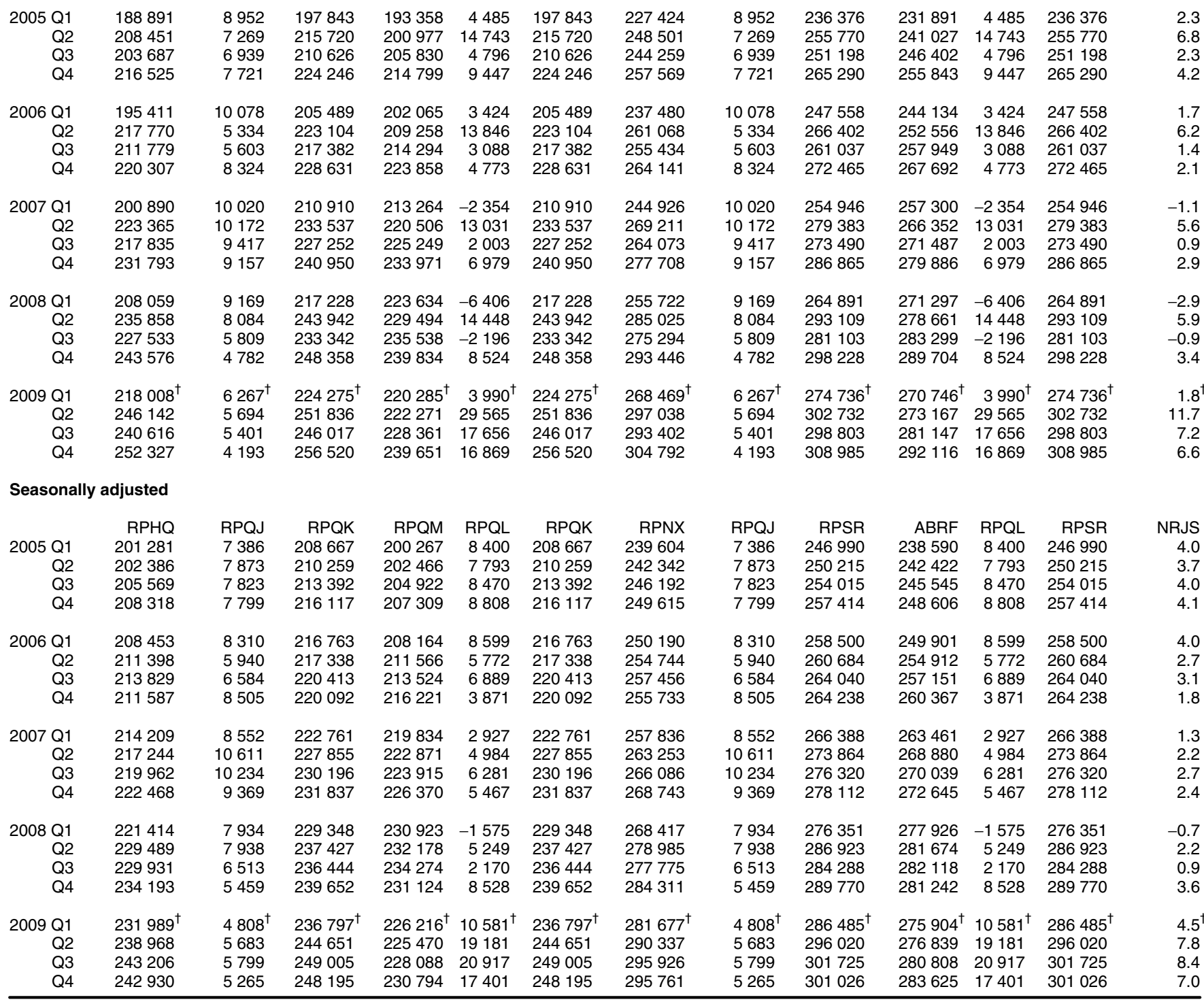




\begin{tabular}{|c|c|c|c|c|c|c|c|c|c|c|c|c|}
\hline & \multicolumn{12}{|c|}{ ACCUMULATION ACCOUNTS } \\
\hline & \multicolumn{12}{|c|}{ CAPITAL ACCOUNT } \\
\hline & \multicolumn{12}{|c|}{ CHANGE IN NET WORTH DUE TO SAVING AND CAPITAL TRANSFERS } \\
\hline & \multicolumn{6}{|c|}{ Changes in liabilities and net worth } & \multicolumn{6}{|c|}{ Changes in assets } \\
\hline & \multicolumn{3}{|c|}{$\begin{array}{l}\text { D.9 Capital transfers } \\
\text { receivable }\end{array}$} & \multicolumn{2}{|c|}{$\begin{array}{l}\text {-D.9 less Capital } \\
\text { transfers payable }\end{array}$} & \multirow[b]{2}{*}{$\begin{array}{c}\text { Total } \\
\text { change in } \\
\text { liabilities } \\
\text { and net } \\
\text { worth }\end{array}$} & \multicolumn{3}{|c|}{ P.5 Gross capital formation } & \multirow{2}{*}{$\begin{array}{r}\text { Acquisitio- } \\
\text { ns less } \\
\text { disposals } \\
\text { of } \\
\text { non-produc- } \\
\text { ed } \\
\text { /financial } \\
\text { assets }\end{array}$} & \multirow[b]{2}{*}{$\begin{array}{r}\text { Net } \\
\text { lending/bo- } \\
\text { rrowing }\end{array}$} & \multirow[b]{2}{*}{$\begin{array}{r}\text { Total } \\
\text { change in } \\
\text { assets }\end{array}$} \\
\hline & $\begin{array}{l}\text { Gross } \\
\text { Saving }\end{array}$ & $\begin{array}{r}\text { Investment } \\
\text { grants }\end{array}$ & $\begin{array}{r}\text { Other } \\
\text { capital } \\
\text { transfers }\end{array}$ & $\begin{array}{r}\text { Capital } \\
\text { taxes }\end{array}$ & $\begin{array}{r}\text { Other } \\
\text { capital } \\
\text { transfers }\end{array}$ & & $\begin{array}{r}\text { Gross fixed } \\
\text { capital } \\
\text { formation }\end{array}$ & $\begin{array}{l}\text { Changes in } \\
\text { inventories }\end{array}$ & $\begin{array}{r}\text { Acquisitio- } \\
\text { ns less } \\
\text { dosposals } \\
\text { of } \\
\text { valuables }\end{array}$ & & & \\
\hline & B.8g & D.92 & D.99 & $-\mathrm{D} .91$ & -D.99 & B. $10.1 \mathrm{~g}$ & P.51 & P.52 & P.53 & K.2 & B.9 & B.10.1g \\
\hline $\begin{array}{l}2001 \\
2002 \\
2003 \\
2004 \\
2005\end{array}$ & $\begin{array}{c}\text { NSSH } \\
43243 \\
35665 \\
40017 \\
29959 \\
33471\end{array}$ & $\begin{array}{l}\text { NSSL } \\
3148 \\
3456 \\
4691 \\
4372 \\
6696\end{array}$ & $\begin{array}{r}\text { NSSM } \\
2639 \\
1869 \\
1956 \\
2831 \\
2755\end{array}$ & $\begin{array}{l}\text {-NSSO } \\
-2396 \\
-2381 \\
-2416 \\
-2881 \\
-3150\end{array}$ & $\begin{array}{r}-N S S Q \\
-1712 \\
-994 \\
-938 \\
-952 \\
-927\end{array}$ & $\begin{array}{r}\text { NSSS } \\
44922 \\
37615 \\
43310 \\
33329 \\
38845\end{array}$ & $\begin{array}{c}\text { NSSU } \\
43457 \\
49764 \\
55226 \\
65394 \\
66659\end{array}$ & $\begin{array}{r}\text { NSSV } \\
199 \\
195 \\
175 \\
126 \\
-261\end{array}$ & $\begin{array}{r}\text { NSSW } \\
374 \\
309 \\
210 \\
215 \\
77\end{array}$ & $\begin{array}{l}\text { NSSY } \\
-152 \\
-176 \\
-210 \\
-276 \\
-320\end{array}$ & $\begin{array}{r}\text { NSSZ } \\
1044 \\
-12477 \\
-12091 \\
-32130 \\
-27310\end{array}$ & $\begin{array}{r}\text { NSSS } \\
44922 \\
37615 \\
43310 \\
33329 \\
38845\end{array}$ \\
\hline $\begin{array}{l}2006 \\
2007 \\
2008 \\
2009\end{array}$ & $\begin{array}{l}25131 \\
19659 \\
14370 \\
68080\end{array}$ & $\begin{array}{l}5256 \\
7243 \\
6177 \\
9049\end{array}$ & $\begin{array}{r}2988 \\
3438 \\
27327 \\
6164\end{array}$ & $\begin{array}{l}-3575 \\
-3867 \\
-3257 \\
-2394\end{array}$ & $\begin{array}{rl}-1 & 157 \\
-1 & 057 \\
-24 & 859 \\
-3 & 426\end{array}$ & $\begin{array}{l}28643 \\
25416 \\
19758 \\
77473\end{array}$ & $\begin{array}{l}74429 \\
81995 \\
67173 \\
50661\end{array}$ & $\begin{array}{r}-16 \\
284 \\
61 \\
-472\end{array}$ & $\begin{array}{l}387 \\
422 \\
529 \\
513\end{array}$ & $\begin{array}{l}-358 \\
-340 \\
-340 \\
-348\end{array}$ & $\begin{array}{r}-45799 \\
-56945 \\
-47665 \\
27119\end{array}$ & $\begin{array}{l}28643 \\
25416 \\
19758 \\
77473\end{array}$ \\
\hline \multicolumn{13}{|c|}{ Not Seasonally adjusted } \\
\hline $\begin{array}{r}2005 \text { Q1 } \\
\text { Q2 } \\
\text { Q3 } \\
\text { Q4 }\end{array}$ & $\begin{array}{r}4485 \\
14743 \\
4796 \\
9447\end{array}$ & $\begin{array}{l}2558 \\
1244 \\
1155 \\
1739\end{array}$ & $\begin{array}{l}671 \\
681 \\
689 \\
714\end{array}$ & $\begin{array}{l}-710 \\
-804 \\
-843 \\
-793\end{array}$ & $\begin{array}{l}-153 \\
-290 \\
-152 \\
-332\end{array}$ & $\begin{array}{r}6851 \\
15574 \\
5645 \\
10775\end{array}$ & $\begin{array}{l}16328 \\
15595 \\
17225 \\
17511\end{array}$ & $\begin{array}{r}-79 \\
-93 \\
-9 \\
-80\end{array}$ & $\begin{array}{r}-17 \\
104 \\
-41 \\
31\end{array}$ & $\begin{array}{l}-75 \\
-79 \\
-81 \\
-85\end{array}$ & $\begin{array}{r}-9306 \\
47 \\
-11449 \\
-6602\end{array}$ & $\begin{array}{r}6851 \\
15574 \\
5645 \\
10775\end{array}$ \\
\hline $\begin{array}{r}2006 \text { Q1 } \\
\text { Q2 } \\
\text { Q3 } \\
\text { Q4 }\end{array}$ & $\begin{array}{r}3424 \\
13846 \\
3088 \\
4773\end{array}$ & $\begin{array}{r}1935 \\
827 \\
1187 \\
1307\end{array}$ & $\begin{array}{l}732 \\
738 \\
749 \\
769\end{array}$ & $\begin{array}{l}-836 \\
-904 \\
-912 \\
-923\end{array}$ & $\begin{array}{l}-310 \\
-172 \\
-174 \\
-501\end{array}$ & $\begin{array}{r}4945 \\
14335 \\
3938 \\
5425\end{array}$ & $\begin{array}{l}17035 \\
17455 \\
19004 \\
20935\end{array}$ & $\begin{array}{r}41 \\
-143 \\
51 \\
35\end{array}$ & $\begin{array}{r}100 \\
175 \\
41 \\
71\end{array}$ & $\begin{array}{l}-88 \\
-90 \\
-91 \\
-89\end{array}$ & $\begin{array}{r}-12143 \\
-3062 \\
-15067 \\
-15527\end{array}$ & $\begin{array}{r}4945 \\
14335 \\
3938 \\
5425\end{array}$ \\
\hline $\begin{array}{r}2008 \text { Q1 } \\
\text { Q2 } \\
\text { Q3 } \\
\text { Q4 }\end{array}$ & $\begin{array}{r}-6406 \\
14448 \\
-2196 \\
8524\end{array}$ & $\begin{array}{l}2543 \\
1286 \\
1150 \\
1198\end{array}$ & $\begin{array}{r}846 \\
852 \\
19868 \\
5761\end{array}$ & $\begin{array}{l}-902 \\
-837 \\
-819 \\
-699\end{array}$ & $\begin{array}{r}-345 \\
-194 \\
-19192 \\
-5128\end{array}$ & $\begin{array}{r}-4264 \\
15555 \\
-1189 \\
9656\end{array}$ & $\begin{array}{l}18337 \\
17413 \\
16299 \\
15124\end{array}$ & $\begin{array}{r}201 \\
3 \\
119 \\
-262\end{array}$ & $\begin{array}{r}75 \\
189 \\
152 \\
113\end{array}$ & $\begin{array}{l}-83 \\
-84 \\
-86 \\
-87\end{array}$ & $\begin{array}{r}-22794 \\
-1966 \\
-17673 \\
-5232\end{array}$ & $\begin{array}{r}-4264 \\
15555 \\
-1189 \\
9656\end{array}$ \\
\hline $\begin{array}{r}2009 \text { Q1 } \\
\text { Q2 } \\
\text { Q3 } \\
\text { Q4 }\end{array}$ & $\begin{array}{r}3990^{\dagger} \\
29565 \\
17656 \\
16869\end{array}$ & $\begin{array}{l}2787^{\dagger} \\
2009 \\
2060 \\
2193\end{array}$ & $\begin{array}{r}2916 \\
924 \\
1371^{\dagger} \\
953\end{array}$ & $\begin{array}{l}-576 \\
-573 \\
-629 \\
-616\end{array}$ & $\begin{array}{c}-2281 \\
-194 \\
-620^{\dagger} \\
-331\end{array}$ & $\begin{array}{r}6836^{\dagger} \\
31731 \\
19838 \\
19068\end{array}$ & $\begin{array}{l}12555^{\dagger} \\
12367 \\
12992 \\
12747\end{array}$ & $\begin{array}{r}-234^{\dagger} \\
-180 \\
23 \\
-81\end{array}$ & $\begin{array}{r}181^{\dagger} \\
98 \\
82 \\
152\end{array}$ & $\begin{array}{l}-87 \\
-87^{\dagger} \\
-87 \\
-87\end{array}$ & $\begin{array}{c}-5579^{\dagger} \\
19533 \\
6828 \\
6337\end{array}$ & $\begin{array}{r}6836^{\dagger} \\
31731 \\
19838 \\
19068\end{array}$ \\
\hline \multicolumn{13}{|c|}{ Seasonally adjusted } \\
\hline $\begin{array}{r}2005 \text { Q1 } \\
\text { Q2 } \\
\text { Q3 } \\
\text { Q4 }\end{array}$ & $\begin{array}{l}\text { RPQL } \\
8400 \\
7793 \\
8470 \\
8808\end{array}$ & $\begin{array}{l}\text { RPVO } \\
2558 \\
1244 \\
1155 \\
1739\end{array}$ & $\begin{array}{r}\text { RPVP } \\
671 \\
681 \\
689 \\
714\end{array}$ & $\begin{array}{r}-R P V S \\
-710 \\
-804 \\
-843 \\
-793\end{array}$ & $\begin{array}{r}\text {-RPVT } \\
-224 \\
-214 \\
-234 \\
-255\end{array}$ & $\begin{array}{r}\text { RPVL } \\
10695 \\
8700 \\
9237 \\
10213\end{array}$ & $\begin{array}{c}\text { RPZW } \\
16645 \\
16032 \\
17153 \\
16829\end{array}$ & $\begin{array}{r}\text { RPZX } \\
-74 \\
-67 \\
-82 \\
-38\end{array}$ & $\begin{array}{r}\text { RPZY } \\
-17 \\
104 \\
-41 \\
31\end{array}$ & $\begin{array}{r}\text { RPZU } \\
-75 \\
-79 \\
-81 \\
-85\end{array}$ & $\begin{array}{r}\text { RPZT } \\
-5784 \\
-7290 \\
-7712 \\
-6524\end{array}$ & $\begin{array}{r}\text { RPVL } \\
10695 \\
8700 \\
9237 \\
10213\end{array}$ \\
\hline $\begin{array}{r}2006 \text { Q1 } \\
\text { Q2 } \\
\text { Q3 } \\
\text { Q4 }\end{array}$ & $\begin{array}{l}8599 \\
5772 \\
6889 \\
3871\end{array}$ & $\begin{array}{r}1935 \\
827 \\
1187 \\
1307\end{array}$ & $\begin{array}{l}732 \\
738 \\
749 \\
769\end{array}$ & $\begin{array}{l}-836 \\
-904 \\
-912 \\
-923\end{array}$ & $\begin{array}{l}-298 \\
-287 \\
-290 \\
-282\end{array}$ & $\begin{array}{r}10132 \\
6146 \\
7623 \\
4742\end{array}$ & $\begin{array}{l}17446 \\
17874 \\
18993 \\
20116\end{array}$ & $\begin{array}{r}-95 \\
59 \\
-203 \\
223\end{array}$ & $\begin{array}{r}100 \\
175 \\
41 \\
71\end{array}$ & $\begin{array}{l}-88 \\
-90 \\
-91 \\
-89\end{array}$ & $\begin{array}{r}-7231 \\
-11872 \\
-11117 \\
-15579\end{array}$ & $\begin{array}{r}10132 \\
6146 \\
7623 \\
4742\end{array}$ \\
\hline $\begin{array}{r}2007 \text { Q1 } \\
\text { Q2 } \\
\text { Q3 } \\
\text { Q4 }\end{array}$ & $\begin{array}{l}2927 \\
4984 \\
6281 \\
5467\end{array}$ & $\begin{array}{l}2446 \\
1232 \\
1592 \\
1973\end{array}$ & $\begin{array}{r}1006 \\
797 \\
810 \\
825\end{array}$ & $\begin{array}{r}-879 \\
-966 \\
-1063 \\
-959\end{array}$ & $\begin{array}{l}-295 \\
-261 \\
-251 \\
-250\end{array}$ & $\begin{array}{l}5205 \\
5786 \\
7369 \\
7056\end{array}$ & $\begin{array}{l}20653 \\
20870 \\
19829 \\
20643\end{array}$ & $\begin{array}{r}145 \\
124 \\
154 \\
-139\end{array}$ & $\begin{array}{r}96 \\
227 \\
82 \\
17\end{array}$ & $\begin{array}{l}-87 \\
-86 \\
-83 \\
-84\end{array}$ & $\begin{array}{l}-15602 \\
-15349 \\
-12613 \\
-13381\end{array}$ & $\begin{array}{l}5205 \\
5786 \\
7369 \\
7056\end{array}$ \\
\hline $\begin{array}{r}2008 \text { Q1 } \\
\text { Q2 } \\
\text { Q3 } \\
\text { Q4 }\end{array}$ & $\begin{array}{r}-1575 \\
5249 \\
2170 \\
8528\end{array}$ & $\begin{array}{ll}2 & 543 \\
1 & 286 \\
1 & 150 \\
1 & 198\end{array}$ & $\begin{array}{r}846 \\
852 \\
19868 \\
5761\end{array}$ & $\begin{array}{l}-902 \\
-837 \\
-819 \\
-699\end{array}$ & $\begin{array}{r}-262 \\
-248 \\
-19245 \\
-5104\end{array}$ & $\begin{array}{l}650 \\
6302 \\
3124 \\
9684\end{array}$ & $\begin{array}{l}18808 \\
17779 \\
16183 \\
14403\end{array}$ & $\begin{array}{r}266 \\
-26 \\
145 \\
-324\end{array}$ & $\begin{array}{r}75 \\
189 \\
152 \\
113\end{array}$ & $\begin{array}{l}-83 \\
-84 \\
-86 \\
-87\end{array}$ & $\begin{array}{r}-18416 \\
-11556 \\
-13270 \\
-4421\end{array}$ & $\begin{array}{l}650 \\
6302 \\
3124 \\
9684\end{array}$ \\
\hline $\begin{array}{r}2009 \text { Q1 } \\
\text { Q2 } \\
\text { Q3 } \\
\text { Q4 }\end{array}$ & $\begin{array}{l}10581^{\dagger} \\
19181 \\
20917 \\
17401\end{array}$ & $\begin{array}{l}2787^{\dagger} \\
2009 \\
2060 \\
2193\end{array}$ & $\begin{array}{r}2916 \\
924 \\
1371^{\dagger} \\
953\end{array}$ & $\begin{array}{l}-576 \\
-573 \\
-629 \\
-616\end{array}$ & $\begin{array}{c}-2263^{\dagger} \\
-247 \\
-675 \\
-241\end{array}$ & $\begin{array}{l}13445^{\dagger} \\
21294 \\
23044 \\
19690\end{array}$ & $\begin{array}{l}13046^{\dagger} \\
12587 \\
12846 \\
12182\end{array}$ & $\begin{array}{c}-470^{\dagger} \\
125 \\
-65 \\
-62\end{array}$ & $\begin{array}{r}181^{\dagger} \\
98 \\
82 \\
152\end{array}$ & $\begin{array}{l}-87 \\
-87^{\dagger} \\
-87 \\
-87\end{array}$ & $\begin{array}{r}775^{\dagger} \\
8571 \\
10268 \\
7505\end{array}$ & $\begin{array}{l}13445^{\dagger} \\
21294 \\
23044 \\
19690\end{array}$ \\
\hline
\end{tabular}




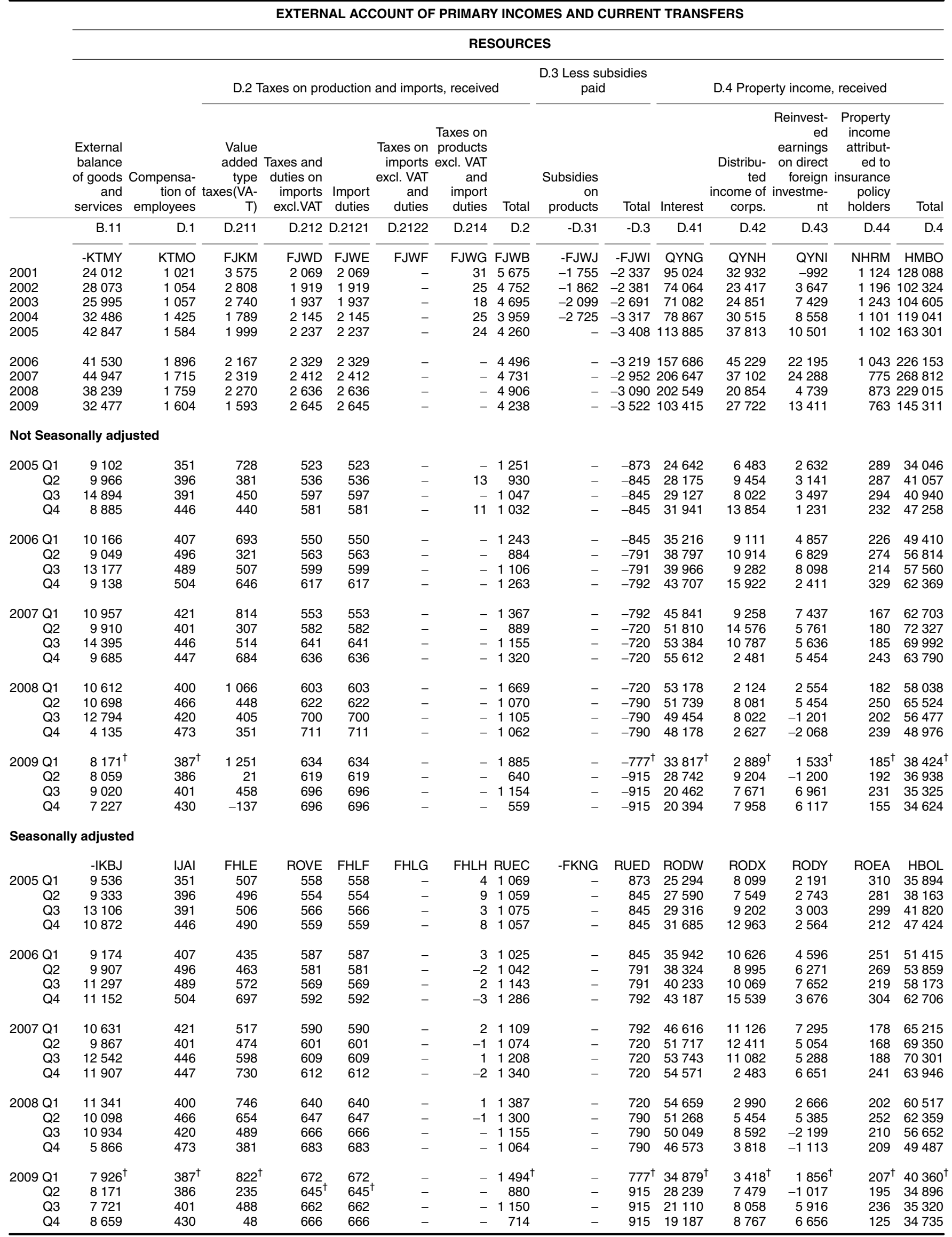




\begin{tabular}{|c|c|c|c|c|c|c|c|c|c|c|}
\hline & \multicolumn{10}{|c|}{ RESOURCES(cont'd) } \\
\hline & \multirow[b]{2}{*}{$\begin{array}{c}\text { Taxes on } \\
\text { income }\end{array}$} & \multirow[b]{2}{*}{$\begin{array}{r}\text { Employees' } \\
\text { social } \\
\text { contributions }\end{array}$} & \multirow{2}{*}{$\begin{array}{r}\text { Social } \\
\text { benefits } \\
\text { other than } \\
\text { social } \\
\text { transfers in } \\
\text { kind }\end{array}$} & \multicolumn{5}{|c|}{ D.7 Other current transfers } & \multirow{2}{*}{$\begin{array}{r}\text { Adj. for the } \\
\text { change in net } \\
\text { equity of } \\
\text { households in } \\
\text { pen. fund }\end{array}$} & \multirow[b]{2}{*}{$\begin{array}{r}\text { Total } \\
\text { resources }\end{array}$} \\
\hline & & & & $\begin{array}{r}\text { Net non-life } \\
\text { insurance } \\
\text { premiums }\end{array}$ & $\begin{array}{r}\text { Non-life } \\
\text { insurance } \\
\text { claims }\end{array}$ & $\begin{array}{r}\text { Current } \\
\text { international } \\
\text { cooperation }\end{array}$ & $\begin{array}{r}\text { Misc. current } \\
\text { transfer }\end{array}$ & $\begin{array}{l}\text { GNP based } \\
\text { fourth own } \\
\text { resource }\end{array}$ & & \\
\hline & D.51 & D.6112 & D.62 & D.71 & D.72 & D.74 & D.75 & - & D.8 & TR \\
\hline $\begin{array}{l}2001 \\
2002 \\
2003 \\
2004 \\
2005\end{array}$ & $\begin{array}{r}\text { FJWM } \\
523 \\
644 \\
444 \\
535 \\
589\end{array}$ & $\begin{array}{r}\text { FJWQ } \\
- \\
- \\
- \\
- \\
-\end{array}$ & $\begin{array}{l}\text { FJKO } \\
1331 \\
1442 \\
1485 \\
1621 \\
1697\end{array}$ & $\begin{array}{r}\text { FJKS } \\
25 \\
19 \\
19 \\
47 \\
16\end{array}$ & $\begin{array}{r}\text { NHRR } \\
3471 \\
3008 \\
2208 \\
3181 \\
6133\end{array}$ & $\begin{array}{l}\text { FJWT } \\
2190 \\
2362 \\
2433 \\
3080 \\
3255\end{array}$ & $\begin{array}{r}\text { FJWU } \\
7222 \\
8878 \\
10610 \\
11631 \\
13354\end{array}$ & $\begin{array}{l}-\mathrm{NMFH} \\
-3858 \\
-5335 \\
-6772 \\
-7549 \\
-8732\end{array}$ & $\begin{array}{r}\text { QZEP } \\
-5 \\
-1 \\
-12 \\
-11 \\
-55\end{array}$ & $\begin{array}{r}\text { NSUK } \\
171216 \\
150174 \\
150848 \\
173678 \\
233573\end{array}$ \\
\hline $\begin{array}{l}2006 \\
2007 \\
2008 \\
2009\end{array}$ & $\begin{array}{r}428 \\
533 \\
1065 \\
428\end{array}$ & $\begin{array}{l}- \\
- \\
- \\
-\end{array}$ & $\begin{array}{l}1765 \\
1866 \\
2029 \\
2251\end{array}$ & $\begin{array}{l}39 \\
50 \\
54 \\
64\end{array}$ & $\begin{array}{l}6831 \\
3320 \\
4478 \\
4088\end{array}$ & $\begin{array}{l}3632 \\
3930 \\
4292 \\
4694\end{array}$ & $\begin{array}{l}13176 \\
13191 \\
13539 \\
15891\end{array}$ & $\begin{array}{r}-8521 \\
-8323 \\
-8423 \\
-10555\end{array}$ & $\begin{array}{r}-9 \\
-37 \\
-2 \\
-42\end{array}$ & $\begin{array}{l}296718 \\
340106 \\
296284 \\
207482\end{array}$ \\
\hline \multicolumn{11}{|c|}{ Not Seasonally adjusted } \\
\hline $\begin{array}{r}2005 \text { Q1 } \\
\text { Q2 } \\
\text { Q3 } \\
\text { Q4 }\end{array}$ & $\begin{array}{l}136 \\
155 \\
140 \\
158\end{array}$ & $\begin{array}{l}- \\
- \\
- \\
-\end{array}$ & $\begin{array}{l}404 \\
408 \\
425 \\
460\end{array}$ & $\begin{array}{l}7 \\
4 \\
4 \\
1\end{array}$ & $\begin{array}{r}1068 \\
1211 \\
3012 \\
842\end{array}$ & $\begin{array}{r}875 \\
347 \\
1123 \\
910\end{array}$ & $\begin{array}{l}4651 \\
2887 \\
3234 \\
2582\end{array}$ & $\begin{array}{l}-3433 \\
-1767 \\
-2102 \\
-1430\end{array}$ & $\begin{array}{r}-2 \\
-49 \\
-2 \\
-2\end{array}$ & $\begin{array}{l}51016 \\
56467 \\
64363 \\
61727\end{array}$ \\
\hline $\begin{array}{r}2006 \text { Q1 } \\
\text { Q2 } \\
\text { Q3 } \\
\text { Q4 }\end{array}$ & $\begin{array}{r}83 \\
87 \\
120 \\
138\end{array}$ & $\begin{array}{l}- \\
- \\
- \\
-\end{array}$ & $\begin{array}{l}406 \\
448 \\
435 \\
476\end{array}$ & $\begin{array}{r}10 \\
15 \\
7 \\
7\end{array}$ & $\begin{array}{l}1676 \\
1868 \\
1695 \\
1592\end{array}$ & $\begin{array}{r}1295 \\
656 \\
910 \\
771\end{array}$ & $\begin{array}{l}3921 \\
2662 \\
3342 \\
3251\end{array}$ & $\begin{array}{l}-2778 \\
-1505 \\
-2171 \\
-2067\end{array}$ & $\begin{array}{l}-4 \\
-3 \\
-1 \\
-1\end{array}$ & $\begin{array}{l}67768 \\
72185 \\
78049 \\
78716\end{array}$ \\
\hline $\begin{array}{r}2007 \text { Q1 } \\
\text { Q2 } \\
\text { Q3 } \\
\text { Q4 }\end{array}$ & $\begin{array}{r}94 \\
104 \\
129 \\
206\end{array}$ & $\begin{array}{l}- \\
- \\
- \\
-\end{array}$ & $\begin{array}{l}461 \\
459 \\
467 \\
479\end{array}$ & $\begin{array}{l}12 \\
11 \\
15 \\
12\end{array}$ & $\begin{array}{r}565 \\
969 \\
755 \\
1031\end{array}$ & $\begin{array}{r}1355 \\
814 \\
859 \\
902\end{array}$ & $\begin{array}{l}3667 \\
2467 \\
3138 \\
3919\end{array}$ & $\begin{array}{l}-2471 \\
-1257 \\
-1914 \\
-2681\end{array}$ & $\begin{array}{r}- \\
-36 \\
- \\
-1\end{array}$ & $\begin{array}{l}80810 \\
87595 \\
90631 \\
81070\end{array}$ \\
\hline $\begin{array}{r}2008 \text { Q1 } \\
\text { Q2 } \\
\text { Q3 } \\
\text { Q4 }\end{array}$ & $\begin{array}{l}270 \\
255 \\
240 \\
300\end{array}$ & $\begin{array}{l}- \\
- \\
- \\
-\end{array}$ & $\begin{array}{l}479 \\
491 \\
501 \\
558\end{array}$ & $\begin{array}{r}13 \\
13 \\
19 \\
9\end{array}$ & $\begin{array}{ll}1 & 219 \\
1 & 124 \\
1 & 089 \\
1 & 046\end{array}$ & $\begin{array}{r}1378 \\
658 \\
991 \\
1265\end{array}$ & $\begin{array}{l}4757 \\
3036 \\
2677 \\
3069\end{array}$ & $\begin{array}{l}-3501 \\
-1770 \\
-1387 \\
-1765\end{array}$ & $\begin{array}{r}- \\
-1 \\
-1 \\
-\end{array}$ & $\begin{array}{l}78115 \\
82544 \\
75522 \\
60103\end{array}$ \\
\hline $\begin{array}{r}2009 \text { Q1 } \\
\text { Q2 } \\
\text { Q3 } \\
\text { Q4 }\end{array}$ & $\begin{array}{c}117^{\dagger} \\
108 \\
99 \\
104\end{array}$ & $\begin{array}{l}- \\
- \\
- \\
-\end{array}$ & $\begin{array}{l}530 \\
551^{\dagger} \\
578 \\
592\end{array}$ & $\begin{array}{l}12 \\
19 \\
23 \\
10\end{array}$ & $\begin{array}{r}952^{\dagger} \\
760 \\
1322 \\
1054\end{array}$ & $\begin{array}{r}1366^{\dagger} \\
869 \\
1096 \\
1363\end{array}$ & $\begin{array}{l}5052 \\
4500 \\
3237 \\
3102\end{array}$ & $\begin{array}{l}-3736 \\
-3173 \\
-1898 \\
-1748\end{array}$ & $\begin{array}{c}-18 \\
-20^{\dagger} \\
-2 \\
-2\end{array}$ & $\begin{array}{l}56101^{\dagger} \\
51895 \\
51338 \\
48148\end{array}$ \\
\hline \multicolumn{11}{|c|}{ Seasonally adjusted } \\
\hline $\begin{array}{r}2005 \text { Q1 } \\
\text { Q2 } \\
\text { Q3 } \\
\text { Q4 }\end{array}$ & $\begin{array}{r}\text { FHLL } \\
149 \\
148 \\
141 \\
151\end{array}$ & $\begin{array}{r}\text { FHLO } \\
- \\
- \\
- \\
-\end{array}$ & $\begin{array}{r}\text { FHLQ } \\
404 \\
408 \\
425 \\
460\end{array}$ & $\begin{array}{l}7 \\
4 \\
4 \\
1\end{array}$ & $\begin{array}{r}\text { FHLS } \\
1104 \\
1207 \\
2945 \\
877\end{array}$ & $\begin{array}{r}\text { FHLT } \\
691 \\
401 \\
1245 \\
918\end{array}$ & $\begin{array}{l}\text { FHLU } \\
3846 \\
3267 \\
3311 \\
2930\end{array}$ & $\begin{array}{r}\text { RNHQ } \\
2628 \\
2147 \\
2179 \\
1778\end{array}$ & $\begin{array}{r}\text { QZED } \\
-2 \\
-49 \\
-2 \\
-2\end{array}$ & $\begin{array}{l}\text { ROVM } \\
52176 \\
53492 \\
63616 \\
64289\end{array}$ \\
\hline $\begin{array}{r}2006 \text { Q1 } \\
\text { Q2 } \\
\text { Q3 } \\
\text { Q4 }\end{array}$ & $\begin{array}{r}90 \\
81 \\
125 \\
132\end{array}$ & $\begin{array}{l}- \\
- \\
- \\
-\end{array}$ & $\begin{array}{l}406 \\
448 \\
435 \\
476\end{array}$ & $\begin{array}{r}10 \\
15 \\
7 \\
7\end{array}$ & $\begin{array}{l}1771 \\
1803 \\
1675 \\
1582\end{array}$ & $\begin{array}{r}1125 \\
782 \\
969 \\
756\end{array}$ & $\begin{array}{l}3187 \\
2983 \\
3361 \\
3645\end{array}$ & $\begin{array}{l}2044 \\
1826 \\
2190 \\
2461\end{array}$ & $\begin{array}{l}-4 \\
-3 \\
-1 \\
-1\end{array}$ & $\begin{array}{l}67761 \\
70622 \\
76882 \\
81453\end{array}$ \\
\hline $\begin{array}{r}2007 \text { Q1 } \\
\text { Q2 } \\
\text { Q3 } \\
\text { Q4 }\end{array}$ & $\begin{array}{r}98 \\
99 \\
135 \\
201\end{array}$ & $\begin{array}{l}- \\
- \\
- \\
-\end{array}$ & $\begin{array}{l}461 \\
459 \\
467 \\
479\end{array}$ & $\begin{array}{l}12 \\
11 \\
15 \\
12\end{array}$ & $\begin{array}{l}612 \\
938 \\
771 \\
999\end{array}$ & $\begin{array}{r}1087 \\
1020 \\
914 \\
909\end{array}$ & $\begin{array}{l}2894 \\
2728 \\
3167 \\
4402\end{array}$ & $\begin{array}{l}1698 \\
1518 \\
1943 \\
3164\end{array}$ & $\begin{array}{r}- \\
-36 \\
- \\
-1\end{array}$ & $\begin{array}{l}81748 \\
85191 \\
89246 \\
83921\end{array}$ \\
\hline $\begin{array}{r}2008 \text { Q1 } \\
\text { Q2 } \\
\text { Q3 } \\
\text { Q4 }\end{array}$ & $\begin{array}{l}274 \\
251 \\
244 \\
296\end{array}$ & $\begin{array}{l}- \\
- \\
- \\
-\end{array}$ & $\begin{array}{l}479 \\
491 \\
501 \\
558\end{array}$ & $\begin{array}{r}13 \\
13 \\
19 \\
9\end{array}$ & $\begin{array}{ll}1 & 175 \\
1 & 182 \\
1 & 096 \\
1 & 025\end{array}$ & $\begin{array}{r}1058 \\
844 \\
1076 \\
1314\end{array}$ & $\begin{array}{l}3739 \\
3393 \\
2917 \\
3490\end{array}$ & $\begin{array}{l}2483 \\
2127 \\
1627 \\
2186\end{array}$ & $\begin{array}{r}- \\
-1 \\
-1 \\
-\end{array}$ & $\begin{array}{l}79663 \\
79606 \\
74223 \\
62792\end{array}$ \\
\hline $\begin{array}{r}2009 \text { Q1 } \\
\text { Q2 } \\
\text { Q3 } \\
\text { Q4 }\end{array}$ & $\begin{array}{l}116^{\dagger} \\
104 \\
106 \\
102\end{array}$ & $\begin{array}{l}- \\
- \\
- \\
-\end{array}$ & $\begin{array}{l}530 \\
551^{\dagger} \\
578 \\
592\end{array}$ & $\begin{array}{l}12 \\
19 \\
23 \\
10\end{array}$ & $\begin{array}{r}969^{\dagger} \\
785 \\
1307 \\
1027\end{array}$ & $\begin{array}{l}1007^{\dagger} \\
1032 \\
1250 \\
1405\end{array}$ & $\begin{array}{l}3885^{\dagger} \\
5151 \\
3472 \\
3383\end{array}$ & $\begin{array}{l}2569^{\dagger} \\
3824 \\
2133 \\
2029\end{array}$ & $\begin{array}{c}-18 \\
-20^{\dagger} \\
-2 \\
-2\end{array}$ & $\begin{array}{l}55891^{\dagger} \\
51040 \\
50411 \\
50140\end{array}$ \\
\hline
\end{tabular}




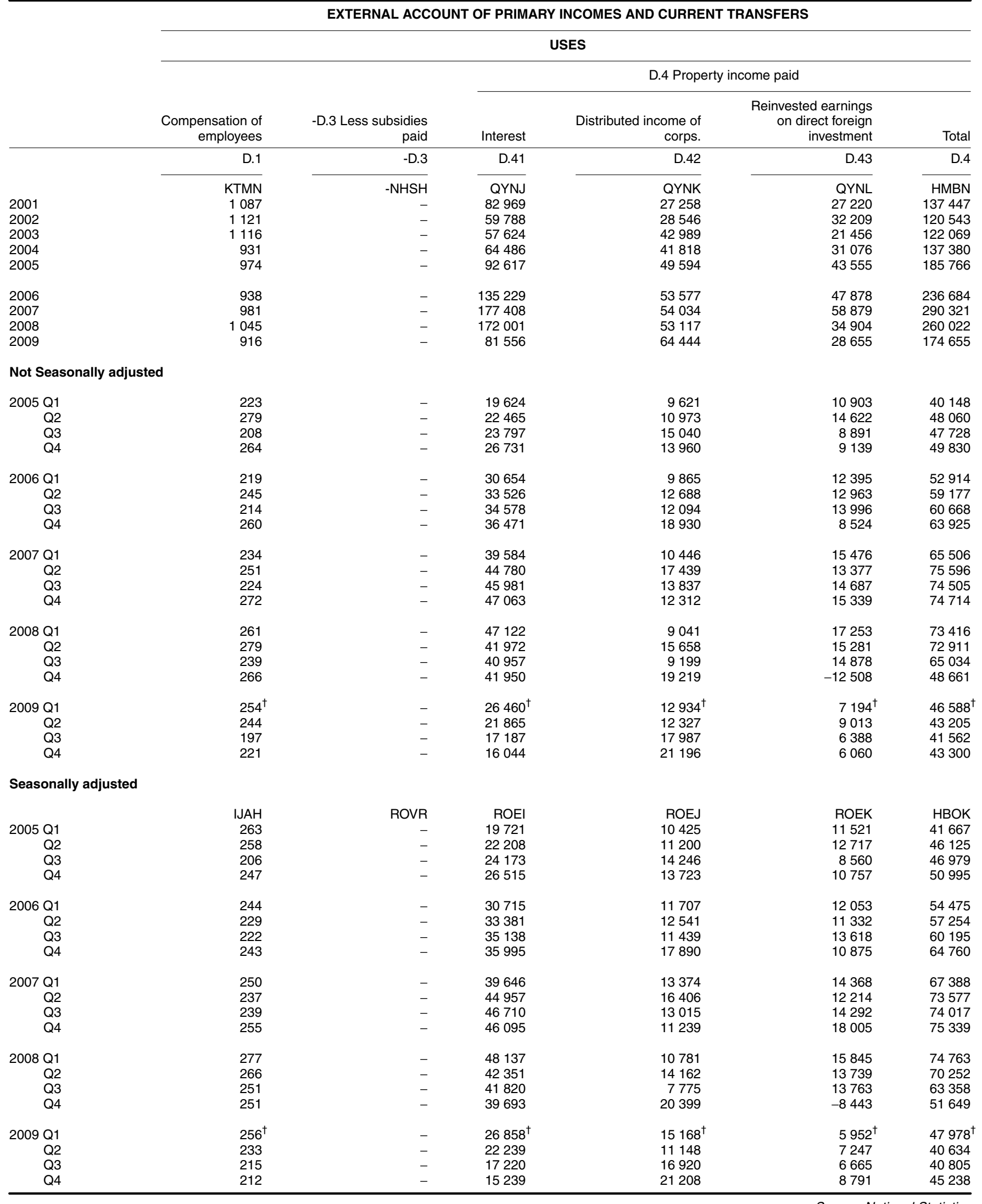


USES(cont'd)

D.7 Other current transfers

\begin{tabular}{|c|c|c|c|c|c|c|c|c|}
\hline & \multirow[b]{2}{*}{ Taxes on income } & \multirow[b]{2}{*}{$\begin{array}{r}\text { Employees' } \\
\text { social } \\
\text { contributions }\end{array}$} & & & & & \multirow[b]{2}{*}{$\begin{array}{r}\text { Current external } \\
\text { balance }\end{array}$} & \multirow[b]{2}{*}{ Total uses } \\
\hline & & & $\begin{array}{r}\text { Net non-life } \\
\text { insurance } \\
\text { premiums }\end{array}$ & $\begin{array}{r}\text { Non life } \\
\text { insurance claims }\end{array}$ & $\begin{array}{r}\text { Current } \\
\text { international } \\
\text { cooperation }\end{array}$ & $\begin{array}{r}\text { Misc. current } \\
\text { transfer }\end{array}$ & & \\
\hline & D.51 & D.6112 & D.71 & D.72 & D.74 & D.75 & B.12 & TU \\
\hline & NHRS & FKAA & NHRX & FJTT & FJWA & NHSI & -HBOG & NSUK \\
\hline 2001 & 398 & 59 & 3471 & 25 & 4568 & 3059 & 21102 & 171216 \\
\hline 2002 & 527 & 77 & 3008 & 19 & 3112 & 3110 & 18657 & 150174 \\
\hline 2003 & 375 & 44 & 2208 & 19 & 3570 & 3140 & 18307 & 150848 \\
\hline 2004 & 482 & 36 & 3181 & 47 & 3673 & 3031 & 24917 & 173678 \\
\hline 2005 & 546 & 14 & 6133 & 16 & 3726 & 3557 & 32841 & 233573 \\
\hline 2006 & 681 & 56 & 6831 & 39 & 3674 & 3973 & 43842 & 296718 \\
\hline 2007 & 608 & 26 & 3320 & 50 & 3676 & 3414 & 37710 & 340106 \\
\hline 2008 & 615 & 68 & 4478 & 54 & 4966 & 3061 & 21975 & 296284 \\
\hline 2009 & 569 & 171 & 4088 & 64 & 5528 & 3056 & 18435 & 207482 \\
\hline
\end{tabular}

Not Seasonally adjusted

$\begin{array}{rr}2005 \text { Q1 } & 121 \\ \text { Q2 } & 137 \\ \text { Q3 } & 13 \\ \text { Q4 } & 15 \\ & \\ 2006 \text { Q1 } & 145 \\ \text { Q2 } & 17 \\ \text { Q3 } & 183 \\ \text { Q4 } & 178 \\ 2007 \text { Q1 } & \\ \text { Q2 } & 151 \\ \text { Q3 } & 14 \\ \text { Q4 } & 156 \\ & 161 \\ 2008 \text { Q1 } & \\ \text { Q2 } & 133 \\ \text { Q3 } & 162 \\ \text { Q4 } & 143 \\ & 177 \\ 2009 \text { Q1 } & \\ \text { Q2 } & 132 \\ \text { Q3 } & 143 \\ \text { Q4 } & 150\end{array}$

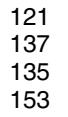

$$
\begin{array}{r}
31 \\
-35 \\
10 \\
8
\end{array}
$$

1068

1211

3012

145

175
183

178

151

140
156

161

133

162

177

$132^{\dagger}$

143
150
144

Seasonally adjusted

2005 Q

Q2

Q3

2006 Q1

Q2

Q4

2007 Q1

Q2
Q4

2008 Q1

Q2
Q3

2009 Q1

Q2
Q3
Q4

FKNI
126
134
138
148

153

171

187
170

159

136

159
154

146

156

145
168

$142^{\dagger}$

143
151

133

$\begin{array}{rr}10 & 1676 \\ 28 & 1868 \\ 8 & 1695 \\ 10 & 1592\end{array}$

1695
1592

\section{5}

969
755

1031

1219

1124

1089
1046

$952^{\dagger}$

$\begin{array}{lr}70 & 952^{\dagger} \\ 31^{\dagger} & 760 \\ 56 & 1322\end{array}$

1322
1054
FKNN

1104

1207

2945
877

1771

1803

1675

1582

612

938

771
999

1175

1182

1096

1025

$969^{\dagger}$

785
1307

1027

1278
681
797
970
1355
624
986
709
1333
500
940
903
1771
769
669
1757

2436
1367
984
741

278
681
797
970

1107

699
813

784

803

1352

1034

759

670
922

922
1063

775

683

638
965

765

$760^{\dagger}$
703
732

732

861
7202

5023

11770

8846

10655

9250

12936

11001

12228

12228
9479

13101

2902

505

6586

7678

7206

$4897^{\dagger}$

5423

6312

1803
51016

56467

64363

61727

67768

72185

78049

78716

80810

87595

90631

81070

78115

82544

75522

60103

$56101^{\dagger}$

51895

51338

48148

\begin{tabular}{rrrr} 
FKNP & FKNQ & -ROVN & ROVM \\
966 & 938 & 7076 & 52176 \\
924 & 1107 & 3769 & 53492 \\
901 & 699 & 11732 & 63616 \\
935 & 813 & 10264 & 64289 \\
950 & 784 & 9367 & 67761 \\
901 & 803 & 9419 & 70622 \\
1124 & 1352 & 12110 & 76882 \\
699 & 1034 & 12946 & 81453 \\
& & & \\
895 & 759 & 11655 & 81748 \\
776 & 670 & 8868 & 85191 \\
1080 & 922 & 12027 & 89246 \\
925 & 1063 & 5160 & 83921 \\
1189 & & & \\
1126 & 775 & 1309 & 79663 \\
844 & 683 & 5912 & 79606 \\
1807 & 638 & 7855 & 74223 \\
$1572^{\dagger}$ & 965 & 6899 & 62792 \\
1836 & & & \\
1235 & 695 & $4209^{\dagger}$ & $55891^{\dagger}$ \\
885 & 730 & 6630 & 51040 \\
& 700 & 5912 & 50411 \\
& 931 & 1684 & 50140 \\
\hline
\end{tabular}

Source: National Statistics 


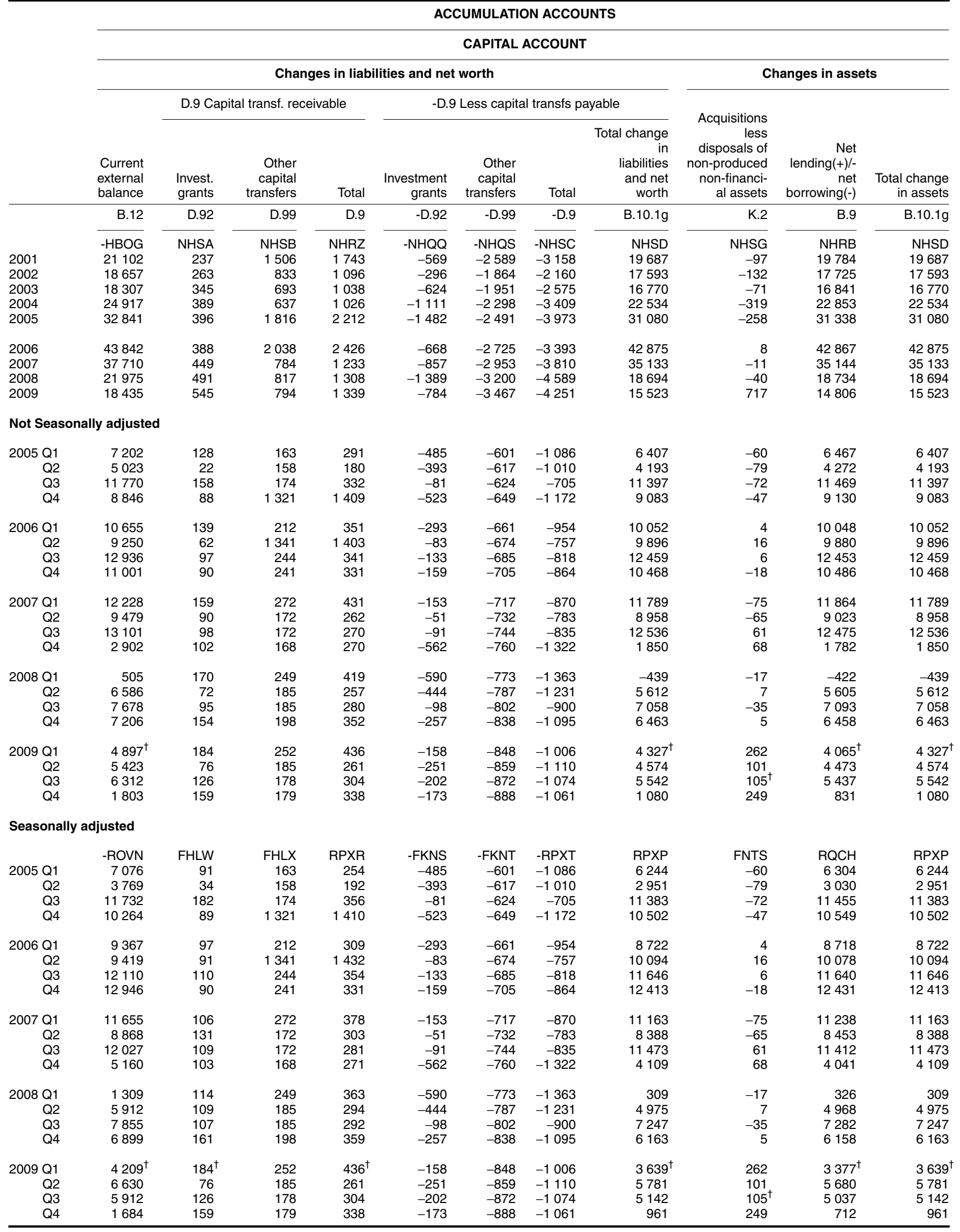

